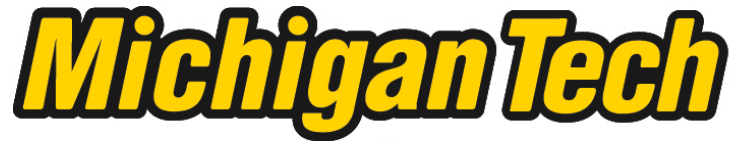 \\ Michigan Technological University Create the Future Digital Commons @ Michigan Tech
}

Dissertations, Master's Theses and Master's Reports - Open

Dissertations, Master's Theses and Master's

Reports

2011

Comprehensive review and application of particle image velocimetry

Michael P. Norconk

Michigan Technological University

Follow this and additional works at: https://digitalcommons.mtu.edu/etds

Part of the Mechanical Engineering Commons

Copyright 2011 Michael P. Norconk

\section{Recommended Citation}

Norconk, Michael P., "Comprehensive review and application of particle image velocimetry", Master's Thesis, Michigan Technological University, 2011.

https://doi.org/10.37099/mtu.dc.etds/392

Follow this and additional works at: https://digitalcommons.mtu.edu/etds

Part of the Mechanical Engineering Commons 


\title{
A COMPREHENSIVE REVIEW AND APPLICATION OF PARTICLE IMAGE VELOCIMETRY
}

\author{
By \\ Michael P. Norconk

\begin{abstract}
A THESIS
Submitted in partial fulfillment of the requirements for the degree of MASTER OF SCIENCE

(Mechanical Engineering)
\end{abstract}

MICHIGAN TECHNOLOGICAL UNIVERSITY

2011

(C) 2011 Michael P. Norconk 
This thesis, "A Comprehensive Review and Application of Particle Image Velocimetry," is hereby approved in partial fulfillment of the requirements for the Degree of MASTER OF SCIENCE IN MECHANICAL ENGINEERING.

Department of Mechanical Engineering - Engineering Mechanics

Signatures:

\begin{tabular}{cl} 
Thesis Advisor & \\
Committee Member & \\
\cline { 2 - 2 } Committee Member & \\
\cline { 2 - 2 } & Dr. Raymond Shaw \\
& \\
Department Chair & \\
& \\
Date & \\
\hline
\end{tabular}




\section{CONTENTS}

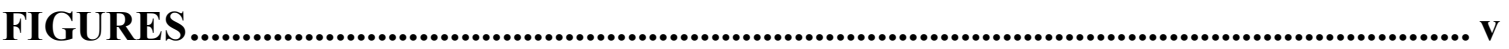

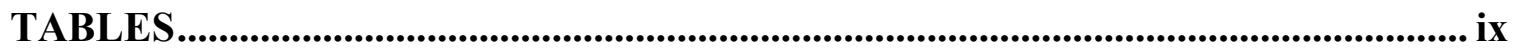

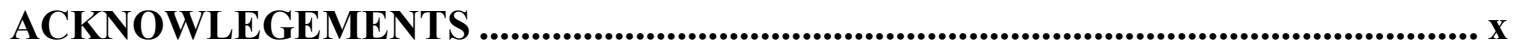

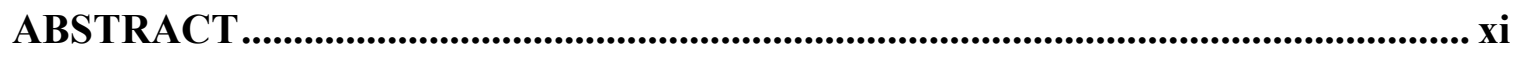

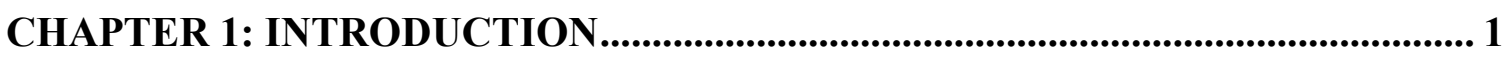

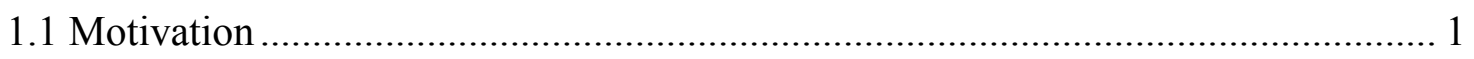

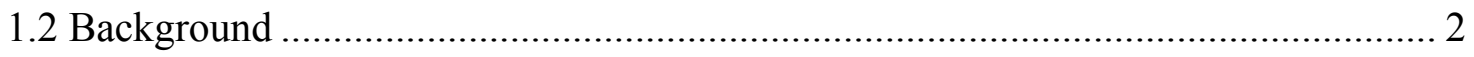

1.3 Objective and Outline of This Thesis Work..................................................... 7

CHAPTER 2: MEASUREMENT PRINCIPLES ................................................ 9

2.1 Measurement Concept................................................................................ 9

2.2 General Test Setup Considerations ........................................................... 14

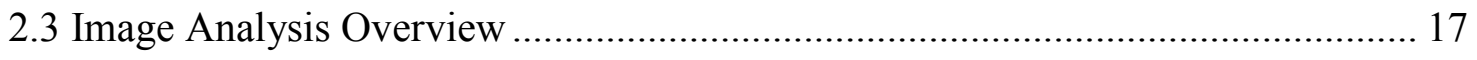

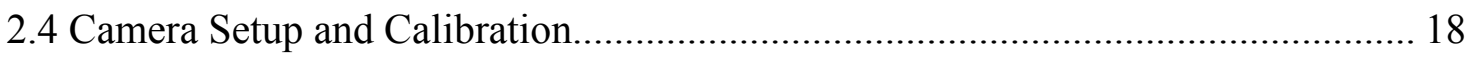

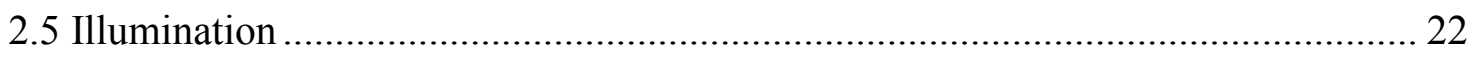

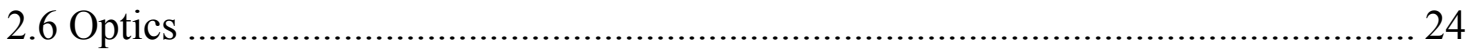

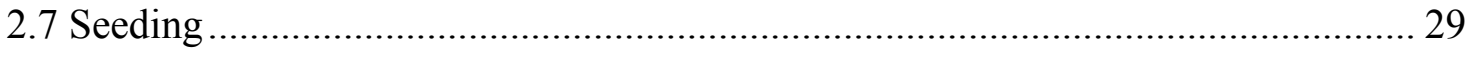

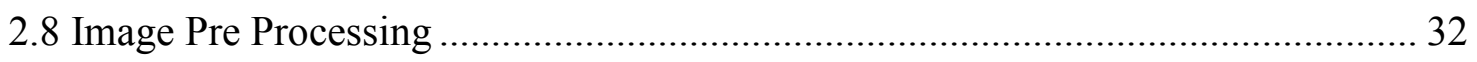

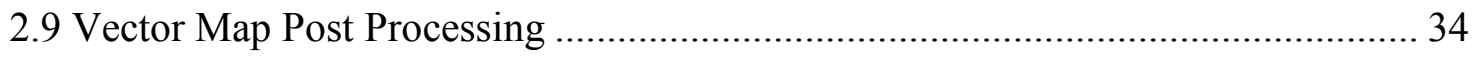


2.10 Test Design Considerations

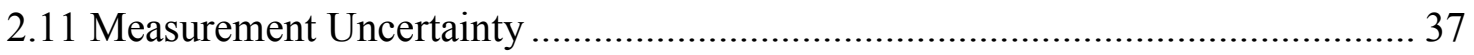

CHAPTER 3: DANTEC DYANMICS SYSTEM ...................................................... 40

3.1 Dantec Dynamics Hardware Overview ........................................................... 40

CHAPTER 4: APPLICATIONS ......................................................................... 45

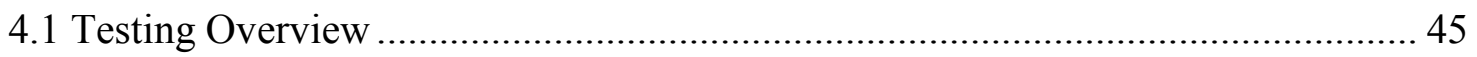

4.2 PIV Measurements in a Flow Channel................................................................ 46

4.3 Stereo PIV of Non-Reacting and Reacting Flows............................................. 58

4.4 Characterization of the AERB Combustion Vessel .......................................... 80

CHAPTER 5: SUMMARY AND FUTURE WORK ..........................................96

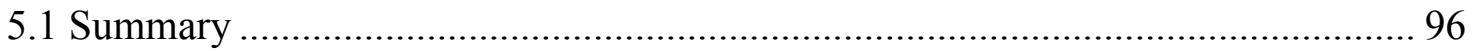

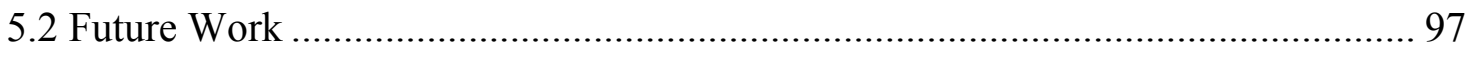

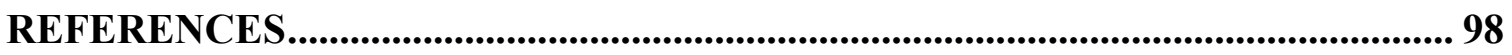

APPENDIX A: PIV OPERATING PROCEDURES ............................................. 100

APPENDIX B: LASER MAINTENANCE .................................................................... 109

APPENDIX C: COMBUSTION VESSEL FLOW PROFILES ............................ 110 


\section{FIGURES}

Figure 2.1 Example of Mie light scattering distribution, where the concentric .15

Figure 2.2 Stereo configurations with coplanar light sheet center axis and cameras. .....16

Figure 2.3 Stereo configurations with light sheet center axis orthogonal to camera .......16

Figure 2.4 Scheimpflug condition for focal and measurement plane alignment ............20

Figure 2.5 Distortion of the fields of view for angled stereo imaging configurations......21

Figure 2.6 Measured velocity relation to true velocity for stereographic PIV. .22

Figure 2.7 Non-diverging width light sheet created by passing a laser beam .25

Figure 2.8 A diverging light sheet configuration using a spherical lens .26

Figure 2.9 Three cylindrical lens configuration forming a thin diverging light sheet.....27

Figure 2.10 Refraction conditions due to optical orientation. .29

Figure 2.11 Matlab Background detection example (Left) raw PIV image (Right) ........34

Figure 3.1 Dantec Dynamics FlowMap system hub. . .40

Figure 3.3 Stereo PIV camera assembly (left) depiction of offset between lens).....

Figure 3.4 Dantec Dyanmic's light guide and sheet optic assembly....

Figure 4.1 Schematic of test hardware and PIV component setup in tumble strip flow..48

Figure 4.2 Tumble strip channel model.

Figure 4.3 Tumble strip flow channel dimensions with bluff body (grey) and PIV........

Figure 4.4 Background image and reference for the PIV camera's field of view. .50 
Figure 4.5 Schematic of a single nozzle aerosol seeding system.

Figure 4.6 Image capturing laser illumination of channel flow downstream .................53

Figure 4.7 CFD and PIV comparison of bluff body flow at $0.8 \mathrm{~m} / \mathrm{s}$ exit velocity. .........54

Figure 4.8 Comparison of numerical and experimental flow magnitude ......................55

Figure 4.9 Vector profiles (left) and contour of flow magnitude (right) for PIV ............55

Figure 4.10 Limited illumination of flow channel for flow measurement. ....................56

Figure 4.11 PIV velocity profile of flow $2.5 \mathrm{~mm}$ above the channel floor.....................57

Figure 4.12 Model gas turbine combustor schematic and swirler injector body............61

Figure 4.13 Schematic of stereo PIV setup.............................................................63

Figure 4.14 Hybrid fluidized bed - cyclone type solid particle seeder for seeding. ........64

Figure 4.15 Time averaged velocity fields for the non-reacting flow of Test 12).........68

Figure 4.19 False-colored chemiluminescence images for Test 2..............................74

Figure 4.20 False-colored rms chemiluminescence images for Test 2........................75

Figure 4.21 Scattering data points for the very initial flame ignition locations for.........77

Figure 4.22 Histogram (frequency) of axial flame ignition locations. .........................78

Figure 4.24 CAD model of the constant volume combustion vessel...........................82

Figure 4.25 Detailed drawings of combustion vessel fan blades................................83

Figure 4.26 Fan blade and spark plug holder positions within the combustion vessel....84

Figure 4.27 Light sheet orientation inside combustion vessel....................................86 
Figure 4.28 MatLab fan calibration plot for single direction fan.

Figure 4.29 MatLab fan calibration plot for bi-directional fan - reverse..........................90

Figure 4.30 MatLab fan calibration plot for bi-directional fan - forward. ......................91

Figure 4.31 Mean velocity profiles near the spark plug for the quiescent flow ..............93

Figure C.1 Mean velocity vector profile for Test $10 \ldots \ldots \ldots \ldots \ldots \ldots \ldots \ldots \ldots \ldots \ldots \ldots \ldots \ldots \ldots \ldots \ldots \ldots \ldots . . .110$

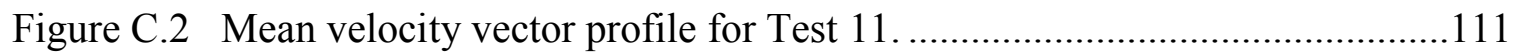

Figure C.3 Mean velocity vector profile for Test $12 \ldots \ldots \ldots \ldots \ldots \ldots \ldots \ldots \ldots \ldots \ldots \ldots \ldots \ldots \ldots \ldots \ldots \ldots \ldots \ldots \ldots . . .111$

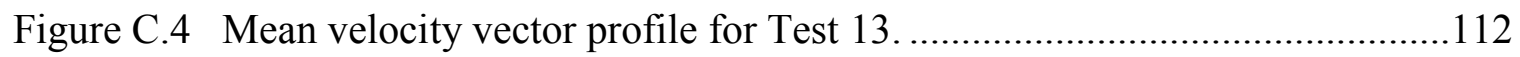

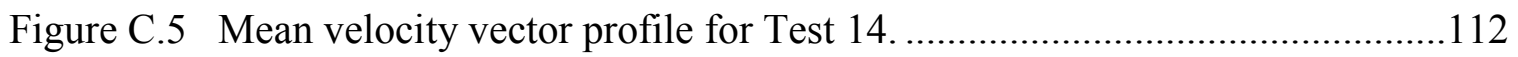

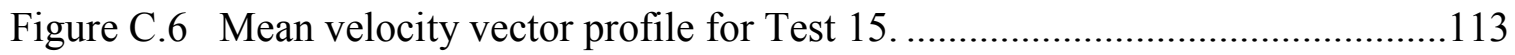

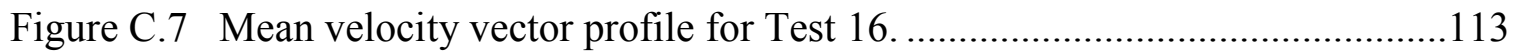

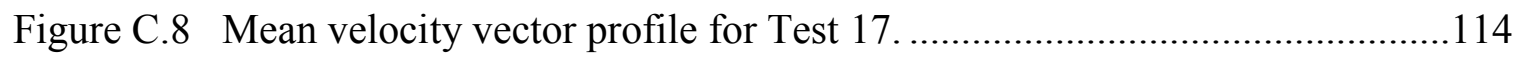

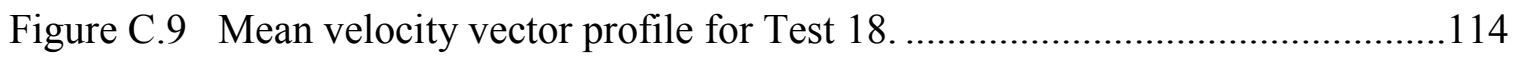

Figure C.10 Mean velocity vector profile for Test $19 \ldots \ldots \ldots \ldots \ldots \ldots \ldots \ldots \ldots \ldots \ldots \ldots \ldots \ldots \ldots \ldots \ldots \ldots . . .115$

Figure C.11 Mean velocity vector profile for Test 20 ..............................................115

Figure C.12 Mean velocity vector profile for Test $22 \ldots \ldots \ldots \ldots \ldots \ldots \ldots \ldots \ldots \ldots \ldots \ldots \ldots \ldots \ldots \ldots \ldots \ldots \ldots . . . .116$

Figure C.13 Mean velocity vector profile for Test $24 \ldots \ldots \ldots \ldots \ldots \ldots \ldots \ldots \ldots \ldots \ldots \ldots \ldots \ldots \ldots \ldots \ldots \ldots \ldots . . . . .116$

Figure C.14 Mean velocity vector profile for Test 26...............................................117

Figure C.15 Mean turbulent velocity profile for Test $10 \ldots \ldots \ldots \ldots \ldots \ldots \ldots \ldots \ldots \ldots \ldots \ldots \ldots \ldots \ldots \ldots \ldots . . .118$ 


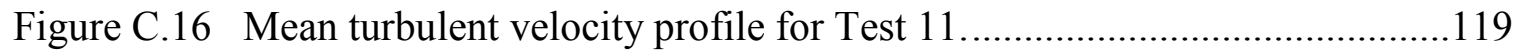

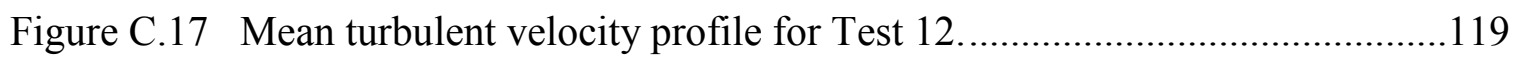

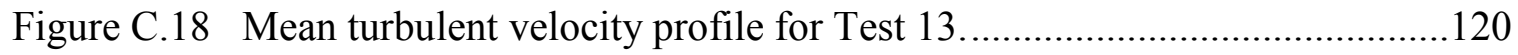

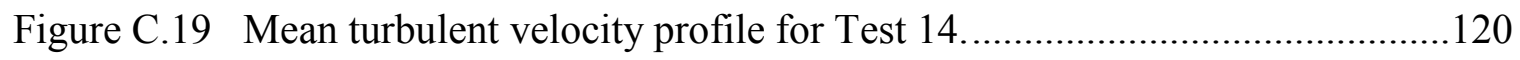

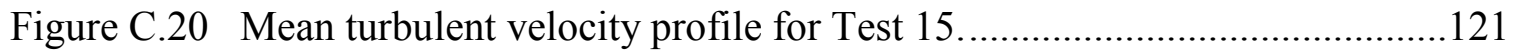

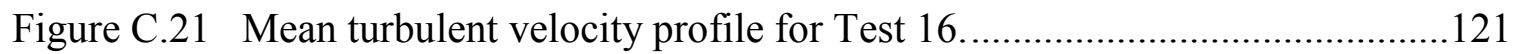

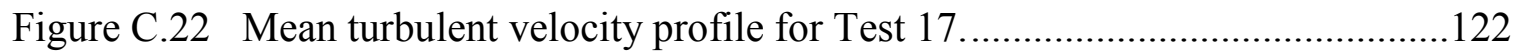

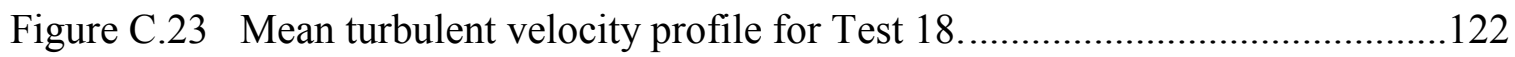

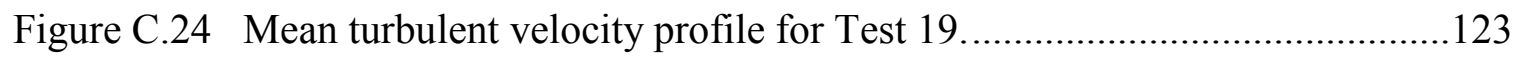

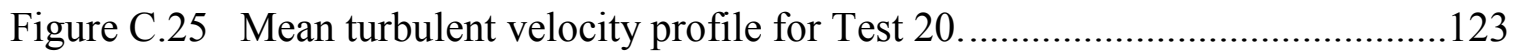

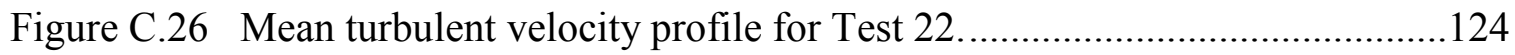

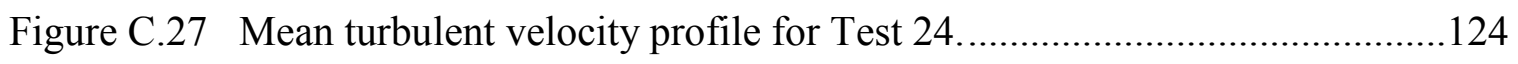

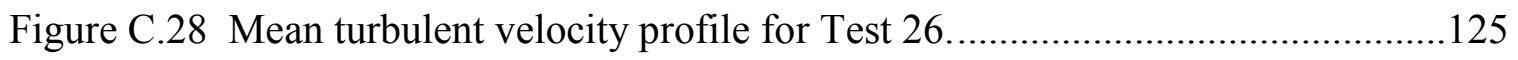




\section{TABLES}

Table 2.1 Key parameters for PIV measurement setup ………………........................13

Table 2.2 Laser alignment trouble shooting guidelines ................................................23

Table 2.3 General characteristics of liquid and solid seeding particles ...........................31

Table 2.4 General characteristics of solid and liquid particle seeders ............................32

Table 3.1 Laser specifications for New Wave Research's Gemini PIV 200 …................41

Table 3.3 Nikon AF Micro-NIKKOR lens specifications ..............................................43

Table 4.1 Test conditions for gas combustor ..............................................................66

Table 4.2 Test matrix for combustion vessel measurements .........................................8 


\section{ACKNOWLEGEMENTS}

First of all, I would like to acknowledge and thank Dr. Lee for providing the opportunity to study under him and be my advisor. It was through his guidance, support, and willingness to share his time, vast knowledge, and expertise in the field of laser diagnostics and combustion that this project was made possible.

I also thank those who have helped support my project throughout its duration in different fashions including my research teammates including: Simon Dezelski, Rajat Aroura, Andrew Ramsey, and Nan Ding. All of whom provided their valuable knowledge and support during different phases of this research.

I would like to thank Pratt \& Whitney's Signature Discipline Group, who supported this research, for both their financial support of this project as well as their guidance and internship opportunity, which served as a predecessor and jumpstart on this research project.

Special thanks to all my colleagues and friends who have made the last two years of my graduate education an enjoyable and memorable chapter in my life. A special thanks to my parents and siblings who have not only supported me throughout this project, but my entire education and life through continual encouragement and motivation. This thesis is dedicated to them. 


\section{ABSTRACT}

For a fluid dynamics experimental flow measurement technique, particle image velocimetry (PIV) provides significant advantages over other measurement techniques in its field. In contrast to temperature and pressure based probe measurements or other laser diagnostic techniques including laser Doppler velocimetry (LDV) and phase Doppler particle analysis (PDPA), PIV is unique due to its whole field measurement capability, non-intrusive nature, and ability to collect a vast amount of experimental data in a short time frame providing both quantitative and qualitative insight. These properties make PIV a desirable measurement technique for studies encompassing a broad range of fluid dynamics applications. However, as an optical measurement technique, PIV also requires a substantial technical understanding and application experience to acquire consistent, reliable results.

Both a technical understanding of particle image velocimetry and practical application experience are gained by applying a planar PIV system at Michigan Technological University's Combustion Science Exploration Laboratory (CSEL) and Alternative Fuels Combustion Laboratory (AFCL). Here a PIV system was applied to non-reacting and reacting gaseous environments to make two component planar PIV as well as three component stereographic PIV flow field velocity measurements in conjunction with chemiluminescence imaging in the case of reacting flows.

This thesis outlines near surface flow field characteristics in a tumble strip lined channel, three component velocity profiles of non-reacting and reacting swirled flow in a swirl stabilized lean condition premixed/prevaporized-fuel model gas turbine combustor operating on methane at 5-7 $\mathrm{kW}$, and two component planar PIV measurements characterizing the AFCL's 1.1 liter closed combustion chamber under dual fan driven turbulent mixing flow. 


\section{CHAPTER $1:$ INTRODUCTION}

\subsection{Motivation}

Particle image velocimetry is a relatively new measurement technique in the field of fluid dynamics measurement. The name particle image velocimetry has only been around for 37 years, first distinguished from laser speckle velocimetry (LSV) in the work by R.J. Adrian in 1984 (1). In its relatively short history, the PIV technique has advanced leaps and bounds due largely in part to the advancement of digital technology. However, the youth of PIV makes it of great interest as there remains a vast potential for improvement in the measurement technique as well as extending its range of application.

Particle image velocimetry (PIV) has become a popular measurement technique in the study of fluid dynamics over the past three decades. The desire to use particle image velocimetry to study fluid dynamics stems from several desirable attributes of the measurement technique including its non-intrusive nature, the ability to collect many flow measurements simultaneously on a flow phenomenon of interest, highly adaptable nature allowing application to many types of flow including gaseous and liquid phases under a broad temperature and pressure range, and especially its ability to provide users insight on both quantitative and qualitative aspects of flow characterization.

In addition to the desirable characteristics of PIV, wide spread implementation of the measurement technique has stemmed from the advancements in digital technology in recent years yielding high resolution digital imaging and relatively inexpensive yet powerful computers for high volume data processing in short. These developments have allowed PIV techniques to be implemented throughout the scientific community in both large and small facilities.

Particle image velocimetry techniques have been employed throughout numerous fields of fluid dynamics research. Applications include automotive, aviation, and structural aerodynamics, as well as the study of hydrodynamics applications such as hull design, 
pipe and channel flow, and mixing characteristics (2). Areas of extensive application also include the field of combustion for the study of liquid sprays, internal combustion flow studies, gas combustor flow studies, and the study of flame dynamics as well the environmental studies ranging from oceanic waves and tides to river hydrology and erosion properties (2).

Of the aforementioned areas of application in fluid dynamics, the area of combustion studies has shown a particularly extensive and successful application of particle image velocimetry. One reason for the extensive application of PIV to combustion studies is the prevalent application of other optical and laser diagnostic techniques deployed in the field including chemiluminescence imaging, species studies using Rayleigh scattering techniques, laser-induced fluorescence (LIF), shadowgraph imaging, and schlieren imaging to name a few. These optical and laser diagnostic techniques can be easily synchronized with particle image velocity measurements, allowing for the coupling of fluid dynamics measurements with combustion characteristics such as flame front tracking, species development and concentration characterization, and emissions measurements. Correlation between these combustion properties and fluid dynamics provides an excellent base for fundamental understanding and implementation of advanced strategies to meet the demands of the combustion industry.

\subsection{Background}

Particle image velocimetry is an experimental flow based measurement technique. The measurement is performed by through adding tracer particles to a flow of interest and recording the motion of the tracer particles to gain insight into the surrounding fluid flow. Particle tracking is accomplished through particle illumination by a laser light source and capture of the particle displacements using one or more cameras. The images, recording particle displacements over a short time interval are then processed using a correlation algorithm to determine the spatial displacement of the particle. With the spatial displacement of the particles and the interval between illumination by the laser, vector maps representing the particle movement and corresponding fluid flow can be generated. 
There are several primary components to any particle image velocimetry measurement systems. First of all, a laser is required for illumination of the tracer particles, typically dual cavity pulse lasers are used for their high power and precise control over the time interval between laser pulses. A seeding system is required for delivery of tracer particles into the flow field and tracer particles can vary widely including gaseous, liquid, and solid particles with sizes spanning six orders of magnitude from the nanometer to millimeter scale. Optical recording is provided by one or more analog or more commonly digital camera and lens systems. Various optics setups are used to manipulate the laser beam into a desirable plane or volume of desirable dimensions. Finally, a synchronization system is required to control the timing between the other hardware components. A computer is also used to perform image processing, provide a user interface and control over test parameters, and save measurement results.

In the field of fluid dynamics there are numerous techniques deployed to measure the velocity characteristics of both gaseous and liquid flows. However, there are two characteristics of PIV measurement techniques that set them apart from other flow velocity measurement techniques. Particle image velocimetry is both a non-intrusive measurement as well as a whole field measurement technique.

Particle image velocimetry benefits from being a non-intrusive measurement technique as this sets it apart from probe based, typically temperature or pressure, measurement techniques form which flow velocities can be derived, this allows for application of PIV measurements to a larger range of flow studies including applications with shocks, near boundary layer measurements, and flow scales down to the micro level where insertion of probe would significantly disrupt the flow structure of interest (3).

As a whole field measurement technique, particle image velocimetry can produce thousands of velocity measurements spanning a flow field simultaneously in a single measurement. Compared to other velocity measurement techniques such as laser Doppler velocimetry (LDV), phase Doppler particle analysis (PDPA), and the aforementioned intrusive probe measurement techniques, where only a single point measurement is made 
at a time. As such particle image velocimetry has the potential for a vast reduction in labor intensity through test time and test setup complexity for the characterization of a flow region while providing higher measurement resolution and data collection that is feasible or practical for point based measurement techniques. This ability of PIV to collect data on flow characteristics over a whole region in short time presents another advantage to the measurement technique. Typically separate techniques are applied to fluid dynamics experiments to acquire quantitative data and qualitative insight into the flow. However, the fine measurement resolution provided by particle image velocimetry makes it capable of providing both comprehensive quantitative analysis of flow characteristics while also providing a qualitative understanding through the visualization and interpretation of resultant vector fields in a single measurement technique.

The term particle image velocimetry covers a broad range measurement techniques, however it typically refers to the simplest PIV measurement, two component planar PIV. A brief overview of the different types of PIV measurements with their unique advantages and limitations are give below.

- Two Component Planar PIV (2D2C PIV): Commonly referred to as 2-D PIV, This is the simplest PIV system consisting of a single camera oriented orthogonal to the measurement plane illuminated by a light sheet. This type of system is capable of measuring the two components of the flow velocity within the planar measurement region defined by the light sheet (4). Advantages of two components planar PIV include low system cost as well as setup, image calibration, and measurement simplicity. Since this setup does not capture the third velocity component, it doesn't provide a complete characterization of the flow velocity profile within the measurement region, as well as the measurements being restricted to a single plane in the flow structure. Additionally, presence of an out of plane velocity component leads to measurement inaccuracies.

- Stereographic PIV (2D3C PIV): Commonly referred to as stereo PIV, is the second simplest form of PIV. While still a two dimensional PIV measurement technique like two component planar PIV where velocity measurement is 
restricted to a planar measurement region, stereo PIV possesses a distinct advantage in the capture of the third, out of plane, velocity component. Capture of the all three components enables full characterization of the velocity within the measurement plane. Stereo PIV operates on the same concept of human vision, where two 2-dimensional 2-component velocity measurements from independent perspectives are combine to form the single two dimensional three component velocity measurement (4). As such, the stereo PIV technique is essentially the combination two synchronized two components planar PIV measurement systems whose two component vector fields are then combine to produce a single three component vector field. Naturally, two PIV cameras are required for this setup with overlapping views of the measurement region. Typically, the cameras are non-orthogonal to the measurement in order to obtain two independent perspectives on the measurement plane. The non-orthogonal camera orientation requires unique setup of the camera-lens system to align the focal plane of the imaging system with the measurement plane of interest through what is known as a Scheimpflug condition, where the camera sensor and lens axis are no longer orthogonal to each other. A calibration target is implemented in this system, allowing for generation of mathematical model to correlate the two fields of view as well as correct the perspective induced in the images from the non-orthogonal orientation of the cameras to the measurement plane. While stereo PIV presents a more complex setup and calibration procedure relative to two component two dimensional PIV, there are several advantages to the system. Not only does the stereo configuration allow for measurement of the out of plane velocity component, allowing for better quantitative and qualitative understanding of the flow velocities, it also eliminates the error introduced into the in-plane velocity components by the out of plane component when not accounted for by two component PIV. Producing a mathematical model of the measurement region also allows for correction of image distortions such as introduced by cylindrical or spherical optical windows for accessing flows. 
- Tomographic PIV (3D3C PIV): Tomographic PIV is a three dimensional three component PIV measurement technique. In this PIV method, a third camera is implemented as well as expanding the laser illuminated light sheet to a substantial thickness for illumination of a small volume. The use of telecentric camera lenses in tomographic PIV limits each camera's field of view to the size of the lens itself, and therefore limiting the size of the measurement volume. In contrast the previously mentioned PIV techniques can be setup with measurement areas orders of magnitude greater than the lens size. The raw images from tomographic PIV systems are processed using the same cross-correlation techniques implemented in planar PIV systems resulting in three dimensional three component vector fields (4). Implementation of tomographic PIV is largely limited due to hardware costs and the skill and experience required for successful setup of the complex measurement configuration.

- Volumetric PIV: Volumetric PIV is a three dimensional PIV measurement method like tomographic PIV, however, it is also much more. While Volumetric PIV differs from tomographic PIV by utilizing a fourth camera in addition to the telecentric lenses, it differs primarily by the means of image processing and flow characteristics measured. Volumetric PIV uses a least squares matching (LMS) based image processing technique to process the four camera images rather than a correlation based algorithm as used by all other PIV systems (2). The LMS processing poses two significant advantages over correlation based processing: it is computationally more efficient and the complete shear tensor simultaneously calculated providing scale, rotation, and shear characteristics of the flow without post processing of the vector profiles (2).

- Time Resolved PIV: Commonly denoted TR-PIV refers to PIV measurements with temporal frequencies in the kilohertz range. While in conventional PIV the flow structures captured in the first and second frame of an image acquisition, the overall measurement acquisition rate lacks the temporal resolution for correlation in the flow structure between consecutive measurements. In contrast, in time resolved PIV, the measurement rate is sufficient to capture correlated transient 
flow structures through consecutive PIV measurements. Time resolved PIV systems typically implement CMOS sensor based cameras compared to CCD sensor cameras used in conventional PIV. The high speed cameras typically have lower resolution and sensitivity compared to conventional PIV cameras. In addition, the corresponding high speed lasers operate with an order of magnitude or lower pulse energy compared to standard dual cavity pulse lasers and typically experience lower beam quality. These two limitations in the hardware components of time resolved PIV systems result in lowering the systems dynamic velocity range.

- Micro PIV: In micro PIV, the PIV setup implements a microscope through which the flow field is imaged with a PIV camera, fluorescent particles are also commonly used to increase the signal strength of the small seeding particles necessitated by the small scale of the flows being measured. While standard PIV techniques frequently result in velocity resolutions on the scale of millimeters, micro PIV vector fields can have a spatial resolution in the micrometer range.

\subsection{Objective and Outline of This Thesis Work}

This thesis presents the investigation of test parameters and considerations regarding the application of both 2-D and 3-D PIV techniques. These considerations include specific test conditions required for the PIV measurement process as well as considerations regarding the measurement technique to optimize the effectiveness and accuracy of the process including test article design considerations, light control considerations, further image processing considerations to improve the cross-correlations processing algorithms and seeding considerations for multiple flow conditions. Meanwhile these considerations were applied to a range of laboratory PIV applications spanning 2-D and 3-D stereographic applications in both non-reacting and reacting flows. This thesis is presented as follows:

- Chapter 2 focuses on a comprehensive overview of both 2-D and 3-D stereographic PIV principles and procedures. This chapter also explores the 
details of the set up and configuration of PIV hardware for successful measurement results.

- Chapter 3 contains specific details of the Dantec Dynamics PIV system used during testing for both 2-D and 3-D stereoscopic PIV configurations.

- Chapter 4 covers the various experiments where PIV was applied over the course of this project, including the motivation, test condition and setup details, measurement results and discussion, conclusions, and insight gained from the application of PIV for each measurement application.

- Chapter 5 provides a summary of the findings and conclusions about the measurement technique. This chapter also includes recommendations to be considered for future PIV applications based on knowledge gained from each test application.

The objective of this study is to gain a substantial understanding of how to apply PIV to a broad range of applications effectively and efficiently, producing consistent and accurate results with minimal trial and error for each test application. This understanding is gained through several means:

- Understanding of the theory and governing principles behind PIV.

- Considerations that should be deployed in the design and setup of an optical measurement system.

- Application of the PIV system to laboratory tests and analysis of the results. 


\section{CHAPTER 2: MEASUREMENT PRINCIPLES}

For successful measurement of fluid flows using a particle image velocimetry measurement technique there are several governing setup characteristics that must be met, and numerous considerations to take into account when designing an experiment and setting up the PIV measurement equipment. This chapter focuses on the fundamental principles of PIV measurement techniques and design consideration for successful PIV measurements.

\subsection{Measurement Concept}

Particle image velocimetry is based on the calculation of particle motion through the imaging of particles using a camera. Understanding of the relationship between the observed particle motion in the measurement field and the corresponding particle image properties of the corresponding image recording is critical to the accurate representation of the flow.

Work by published by Keane and Adrian in 1992 (5), established a dimensionless number critical to producing accurate PIV measurements. The dimensionless number, $N_{I} F_{I} F_{O}$, called the image density was shown to have a direct correlation to the probability of making a valid PIV flow measurement through image processing using auto and cross correlation techniques (5). The dimensionless number is based on the calculation from the number of particle images captured in an interrogation region $\left(N_{I}\right)$, and fractions of particles that remain within the interrogation region and light sheet during their displacement from the first to second exposure measurement due to displacement between measurements due to in plane $\left(F_{I}\right)$ and out of plane $\left(F_{O}\right)$ motion. With proper measurement conditions, the number of lost image pairs is minimized and the dimensionless number is essentially represented by the number of particle images captured in an interrogation region. It was found that for an image density greater than ten, the probability of making a valid PIV measurement approaches unity (1). Later work by Hain and Kahler (6), took this work further, showing that the correlation peak 
amplitude, $R_{D}$, was proportional to the dimensionless number developed by Keane and Adrian. Hain and Kahler took the expression for correlation peak amplitude further to account for both in and out of plane velocity gradients within the interrogation region as well as curvature and acceleration within the interrogation region, the presence of which diminishes the correlation peak amplitude and image density value (6). Based on the development of these two dimensionless numbers and their influence on the probability of making valid PIV measurements, test setup guidelines have been developed to maximize these values.

For PIV measurements, the number of images captured per interrogation region should exceed the value of ten, as established by Keane and Adrian to virtually eliminate the probability of an invalid correlation calculation.

$$
N \geq 10
$$

For the number of particle images per interrogation region to accurately represent the probability predicting functions $N_{I} F_{I} F_{O}$ and $R_{D}$, the particle pair loss, gradient, curvature, and acceleration parameters for flow inside the interrogation region must be minimized. This can be accomplished by adhering to the following series of setup parameters.

The maximum velocity gradient within an interrogation region should be low enough to maintain the shape of the particle group within an interrogation region between frames. An acceptable gradient in the flow velocity for an interrogation region can be expressed as:

$$
\frac{M|\Delta u| \Delta t}{d_{I}}<0.05
$$


Where $M$ is the magnification factor, $\Delta \mathrm{u}$ is the velocity gradient within the interrogation region, $\Delta \mathrm{t}$ is the time separation between frames, and $d_{I}$ is the length of interrogation region the image sensor.

The magnification factor produced by the camera lens can be measured by the ratio of the interrogation region size on the image sensor to the size of the region in the measurement plane represented by the interrogation region.

$$
M=\frac{d_{I}}{d_{1}}
$$

The number of lost pairs between frames can be reduced by minimizing particle displacement within the interrogation region. However, the significance of displacement measurement errors are reduced with large particle displacements. As a compromise, a target particle displacement between frames relative to the interrogation region size is given by the following equation where $\Delta X$ is the particle displacement in the flow field between frames is and $d_{1}$ is the length of the interrogation region projected into the measurement plane:

$$
\frac{|\Delta X|}{d_{1}}<0.25
$$

The same target ratio is established as a compromise for out of plane particle loss and measurement accuracy where $\Delta Z$ is the transverse particle displacement between frames and $\Delta Z_{0}$ is the laser light sheet thickness.

$$
\frac{|\Delta Z|}{\Delta Z_{0}}<0.25
$$


When using auto correlation processing for doubly exposed single frame images, the minimum particle displacement is critical. Displacement of particle image on CCD chip should be greater than twice the particle's image diameter to avoid particle superpositioning. If the particles are super-positioned on a doubly exposed image, the center of the particle at each exposure time is indeterminable. Therefore, the minimum acceptable velocity can be established from the particle displacement give the following equitation where $d_{\zeta}$ is the particle image diameter

$$
\frac{M|\Delta X|}{2}<d_{\zeta}
$$

$d_{\zeta}=$ particle image diameter

The particle image diameter can be defined by the magnification factor $M$, and the actual particle diameter $d_{p}$.

$$
d_{\zeta}=M d_{p}
$$

The particle image diameter should be greater than three times the cell size on the image sensor, allowing for a sub pixel calculation the particle's center based on a Gaussian distribution of the light scattered by the particle.

A summary of the parameters relevant to the setup of PIV measurements is shown in Table 2.1 (7). For each parameter, a description, units, and the means for determining the value of the parameter are given. 
Table 2.1

Key parameters for PIV measurement setup.

\begin{tabular}{|c|c|c|c|c|c|}
\hline Parameter & Description & Units & Fixed & Calculated & Adjustable \\
\hline $\mathrm{N}$ & $\begin{array}{l}\text { Number of particles in } \\
\text { an interrogation spot }\end{array}$ & - & & $\mathrm{X}$ & \\
\hline$\Delta t$ & $\begin{array}{l}\text { Time interval between } \\
2 \text { pulses }\end{array}$ & time & & & $X$ \\
\hline$\Delta Z_{0}$ & Laser beam thickness & length & $\mathrm{X}$ & & \\
\hline $\mathrm{d}_{\mathrm{I}}$ & $\begin{array}{l}\text { Size of } 1 \text { interrogation } \\
\text { spot Image (on the } \\
\text { camera) }\end{array}$ & length & $\mathrm{X}$ & & \\
\hline $\mathrm{d}_{1}$ & $\begin{array}{l}\text { Size of interrogation } \\
\text { spot for the observed } \\
\text { area (in the flow) }\end{array}$ & length & & & $\mathrm{X}$ \\
\hline M & $\begin{array}{l}\text { Camera Lens } \\
\text { Magnification }\end{array}$ & - & & $\mathrm{X}$ & \\
\hline $\mathrm{d}_{\mathrm{p}}$ & Particle diameter & length & & & $\mathrm{X}$ \\
\hline$d_{\zeta}$ & $\begin{array}{l}\text { Particle image } \\
\text { diameter }\end{array}$ & length & & $\mathrm{X}$ & \\
\hline $\mathrm{u}$ & $\begin{array}{l}\text { Particle local velocity } \\
\text { for an interrogation } \\
\text { spot }\end{array}$ & $\begin{array}{l}\text { length/ } \\
\text { time }\end{array}$ & $\begin{array}{c}\mathrm{X} \\
\text { (estimated) }\end{array}$ & & \\
\hline $\mathrm{W}$ & $\begin{array}{l}\text { Particle transverse } \\
\text { velocity for an } \\
\text { interrogation spot }\end{array}$ & $\begin{array}{l}\text { length/ } \\
\text { time }\end{array}$ & $\begin{array}{c}\mathrm{X} \\
\text { (estimated) }\end{array}$ & & \\
\hline$\Delta u$ & $\begin{array}{l}\text { Difference between } \\
\text { the highest and lowest } \\
\text { velocity of the flow } \\
\text { for an interrogation } \\
\text { spot }\end{array}$ & $\begin{array}{l}\text { length/ } \\
\text { time }\end{array}$ & $\begin{array}{c}\mathrm{X} \\
\text { (estimated) }\end{array}$ & & \\
\hline$\Delta X$ & $\begin{array}{l}\text { Mean particle } \\
\text { displacement for an } \\
\text { interrogation spot } \\
\text { (X-Y plane) }\end{array}$ & length & & $\mathrm{X}$ & \\
\hline$\Delta Z$ & $\begin{array}{l}\text { mean particle } \\
\text { displacement for an } \\
\text { interrogation spot } \\
\text { (Z Axis) }\end{array}$ & length & & $\mathrm{X}$ & \\
\hline $\mathrm{C}$ & Seeding Concentration & $\begin{array}{c}\# \\
\text { particles } \\
/ \\
\text { volume }\end{array}$ & & $\mathrm{X}$ & \\
\hline
\end{tabular}




\subsection{General Test Setup Considerations}

\section{Acquisition Rate}

There are several limiting factors to the PIV systems regarding total acquisition rate. The ability of the PIV cameras in dual frame mode limit to capture and transmit images to the system hub limits the acquisition rate to $5.6 \mathrm{~Hz}$. However, this acquisition rate can only be sustained for a finite amount of time. The further limiting factor to the system's acquisition rate is the rate at which the system hub can transfer PIV images to the control computer. The system hub utilizes a LIFO, which stands for Last In First Out, memory buffer. This allows for images from the camera to come in to the system hub faster than they can be transferred to the control computer to be saved on a hard drive for a finite time. If the buffer is filled, the system hub will freeze up halting image acquisition. Therefore, measurements can be acquired in two ways rapid acquisition of a low number of PIV images, or slow acquisition of a large number of images. The exact number of images that can be captured without overloading the buffer depends on the image acquisition rate. Note that in stereo PIV mode, half the number of measurements can be taken since there are two images and four frames for each measurement compared to a 2D PIV setup at the same acquisition rate.

\section{Stereo-PIV Configuration Considerations}

In the case of stereo PIV extra considerations are necessary. Given in stereo PIV multiple cameras are in use and at least one camera is not orthogonal to the light sheet plane, extra care is required to prevent reflections of the light sheet into the cameras' fields of view. The orientation of the cameras must also be considered carefully for good measurement results due to the light scattering properties of the seeding particles. In the case of most PIV measurements, where the seeding particles are typically larger in diameter than the wavelength of the laser light, Mie scattering light properties are dominant. For Mie scattering, the intensity of scattered light is highly biased to forward scatter as shown in by the diagram of Figure 2.1 below. In the case of stereo PIV, the 
intensity of light forward scattered by an illuminated particle can be several orders of magnitude greater than the back scatter intensity from the particle. For this reason, stereo camera setups requiring such a configuration are avoided when possible, as it is difficult to balance image quality between the cameras.

Figure 2.2 depicts several examples of camera-light sheet configurations for stereo PIV acquisition where the center axis of the light sheet and the camera viewing axis are all coplanar. In configuration in A, both cameras capture a strong forward scatter signal. In configuration $\mathrm{B}$, the upper camera receives a backscatter light signal while the lower camera receives a much stronger forward scatter signal, resulting in an imbalance in image quality which should be avoided. In configuration $\mathrm{C}$, both cameras capture a back scatter signal, signal strength is weaker compared to that captured in configuration A, but remains balanced for the two cameras.

For greater versatility in camera positioning, it is typically beneficial to orient the light sheet with a center axis normal to the plane of the cameras as seen in the example configurations of Figure 2.3. This allows for side scatter light to be captured by the cameras for any position around the light sheet axis. The side scatter light strength is also a compromise between the intense forward scatter and weak back scatter particle illumination.

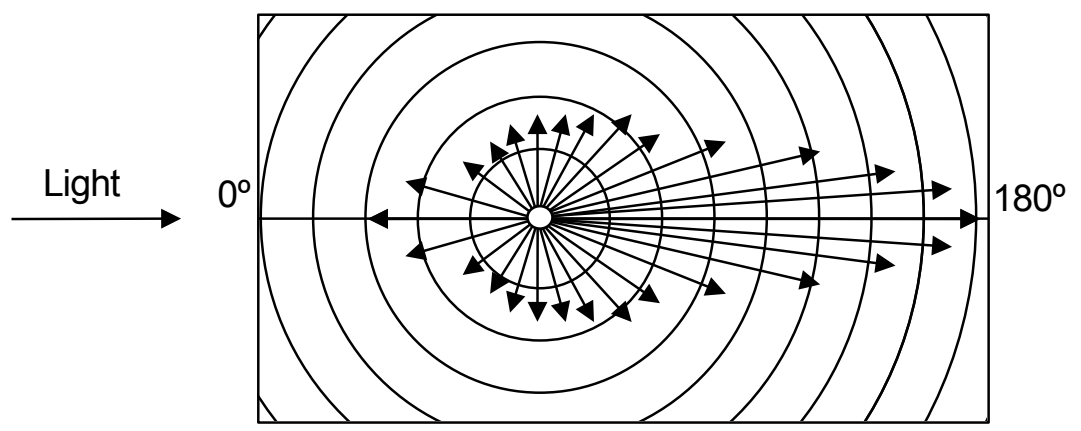

Figure 2.1 Example of Mie light scattering distribution, where the concentric rings represent increasing orders of magnitude in light scatter intensity. 


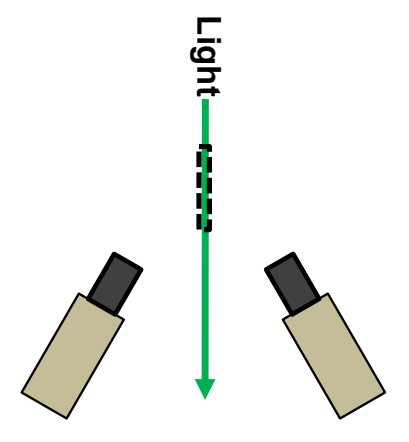

A

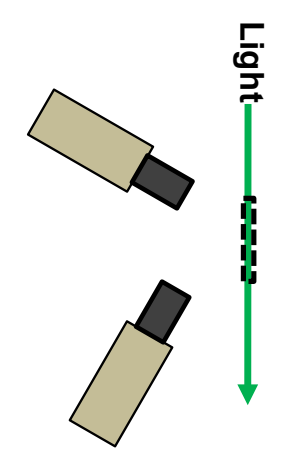

B

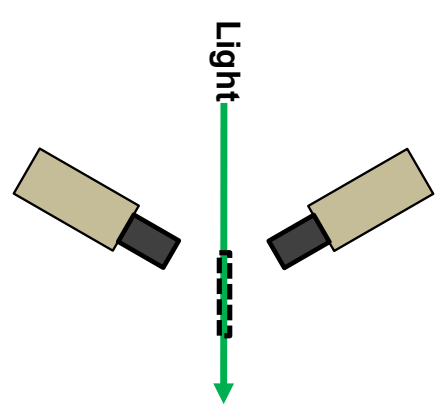

C

Figure 2.2 Stereo configurations with coplanar light sheet center axis and cameras axis $A$, forward-forward scatter $B$, forward-back scatter and $C$, back-back scatter.

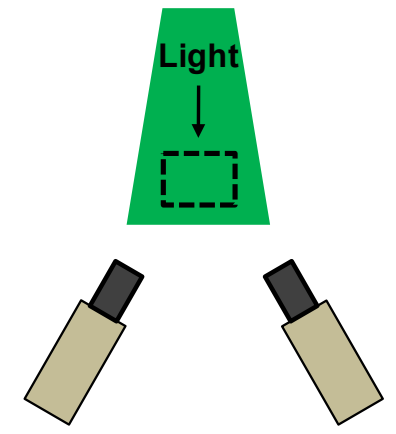

A

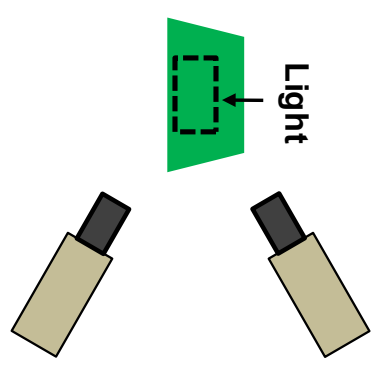

B

Figure 2.3 Stereo configurations with light sheet center axis orthogonal to camera axis plane $A$, Non-bisecting configuration $B$, Bisecting configuration. 


\subsection{Image Analysis Overview}

\section{Image Analysis using Auto or Cross Correlation}

Processing of the raw images from PIV cameras into a field of velocity measurements is achieved through a correlation processing. The type of processing is depended on the method by which the images were collected. During early development of PIV, cameras did not exist that were capable of taking two separate image frames microseconds apart, rather, a single image was taken composed of two exposures to the seeded flow illuminated by the laser light. With doubly exposed single frame images, there are two positions of a particle represented in the image; however, the first and second exposure positions cannot be readily distinguished from each other leaving direction of flow is ambiguous. However, with the advent of cameras designed for PIV measurements, first developed by Kodak, separate frames for the first and second exposure were made possible while maintaining an identical spatial representation (1). With separate frames, the particle exposure order is easily defined, eliminating the ambiguity of flow direction. With the advent of double frame imaging, auto-correlation can be substituted with crosscorrelation techniques. The use of double frame single exposed images and crosscorrelation processing was a milestone in the advancement of PIV measurements due to the increase in the measurement technique's dynamic velocity range from this advancement.

The dynamic velocity range for PIV measurements is defined as the ratio between the maximum measureable velocities to the minimum measureable velocity. With each exposure represented on a separate image frame, the inability to define the particle due to particle image super-positioning at low displacements was eliminated. This drastically reduced the minimum measureable velocity in an interrogation region, and increased the dynamic velocity range from 5 to 10 for double exposure image auto-correlation to a range of 100-300 for the double frame single exposure images and cross-correlation measurement technique (1). This leap in dynamic velocity range was important for several reasons. First, with auto correlation the range of measureable velocity is simply 
too small for application in advanced fluid dynamics studies. Second, increasing the dynamic velocity range reduces the how critical the setup parameters previously covered are to achieving a valid flow measurement. This enables the application of PIV measurements to situations where there is little knowledge of the flow characteristics going into the experiment and still be able to make valid measurements with the system. Both aspects make the measurement technique more desirable and applicable to experimental measurements.

\section{Validation Parameters}

An appropriate range validation can always be applied to the measurements with the threshold level set by inspection with a safety margin for fluctuations in the flow to eliminate spurious correlations resulting in excessively high or low velocities with little to no drawbacks.

Other validation routines including peak validation and local moving average validations can be applied on a case by case bases using inspection for improvement in the desired measurement region.

\subsection{Camera Setup and Calibration}

\section{Field of View}

The camera's field of view should be limited to the region in which measurements are required whenever possible, allowing for maximum spatial resolution. A lower scale factor results in a greater number of pixels representing an individual seed particle image on the camera's CCD and more accurate subpixel calculation of the particle's center. The interrogation region size is also linked to the field of view. When selecting an appropriate interrogation region size, there are a few critical assumptions that are made. The most critical assumption is that the flow field within each interrogation region is uniform. This is a critical criterion for two reasons. One, the correlation processing makes this assumption and looks for the highest probability transform of the particle 
images from one location to another over the entire interrogation region. Second, each interrogation region is represented as a single vector in the velocity map output result.

\section{2-Component Planar PIV}

Two component planar PIV benefits from a simple imaging setup and calibration procedure compared to other forms of PIV. The camera is positioned orthogonal to the measurement plane at a distance suitable for the desired field of view. The camera lens $f$ stop setting should be adjusted for the depth of field to encompass the measurement plane

and satisfy the conditioned outlined at the beginning of this chapter. Focusing and determination of the magnification factor for the imaging setup can be achieved easiest by placing an object of known dimension in the measurement plane, typically a small ruler or like object works well.

\section{3-Component Stereo PIV}

A PIV system configured for stereographic measurement does not enjoy the same simple setup and calibration procedure as that of two component PIV systems. In a stereographic configuration, the two cameras are not oriented orthogonal to the measurement plane, and therefore the focal plane is not aligned with the measurement plane under normal circumstances. This issue is overcome through the implementation of camera-lens configuration known as a Scheimpflug condition, where the lens axis is no longer orthogonal to the image sensor. Instead, the lens is angled relative to the image sensor. A Scheimpflug condition for aligning the focal planes and measurement plane for a stereo PIV measurement are shown in Figure 2.4.

Here, $d_{0}$ is represents the distance between lens center and the center of the measurement region, the distance from the image sensor to the center of the lens is denoted by $d_{i}$, and $\theta$ is the angle between the camera lens and the measurement plane. The angle $\alpha$ is the offset angle between the camera lens axis and the image sensor, which can be numerically determined by: 


$$
\alpha=\tan ^{-1}\left[\left(f_{\text {lens }} * \tan \theta\right) /\left(d_{0}-f_{\text {lens }}\right)\right]
$$

With the cameras at an angle to the measurement, significant perspective is introduced to the camera images, and therefore a gradient in magnification factor, that must be corrected for. Correction for the introduced perspective as well as correlation between the two camera images is typically provided by means of a calibration target as sown in Figure 2.5. The calibration target consists of a grid of dots with known size and spacing, within the grid, there is a uniquely sized dot representing the origin point and a few surrounding dots of unique size used to define the in plane axis. By traversing the target through the measurement plane and taking images from both cameras at each known out of plane, z, location, a mathematical model can be generated to align the two sets of images, define the area of overlap between the two images giving the valid region for PIV measurements, and correct the perspective distortions.

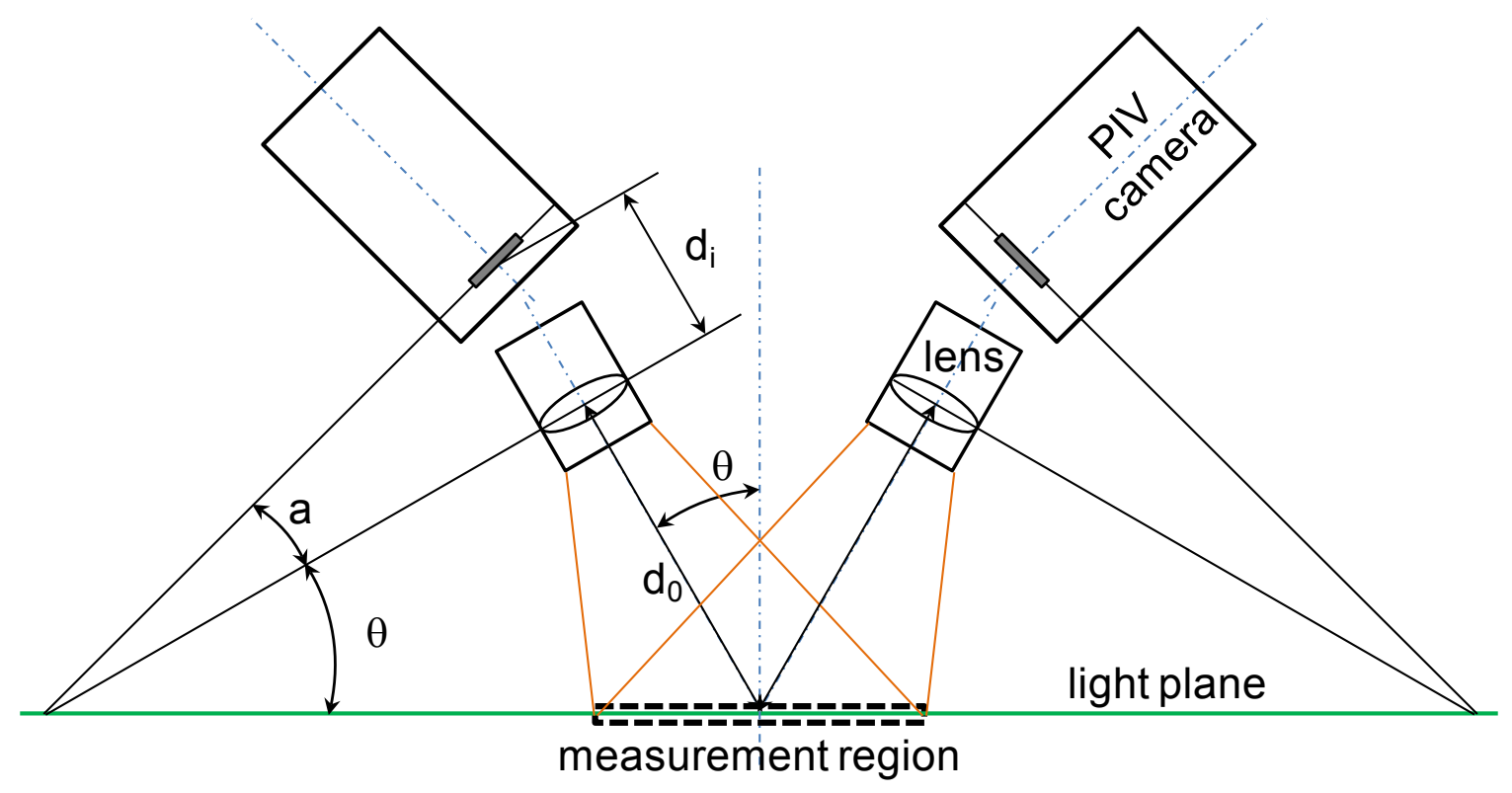

Figure 2.4 Scheimpflug condition for focal and measurement plane alignment in a stereo PIV setup. 


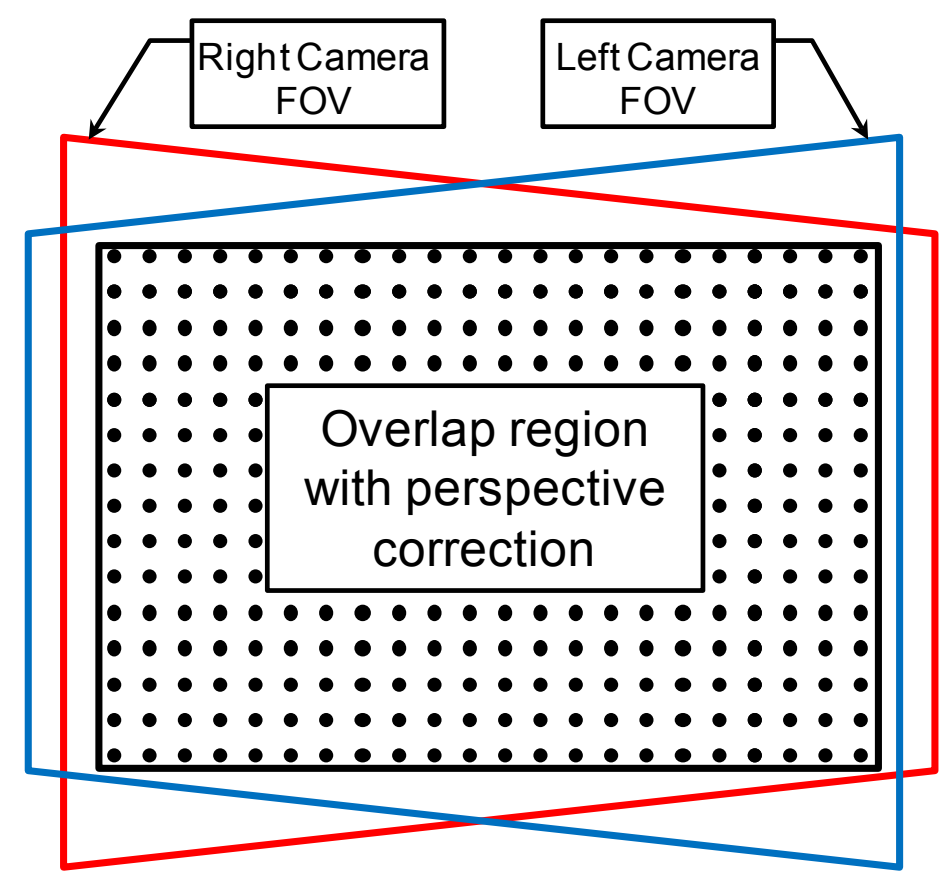

Figure 2.5 Distortion of the fields of view for angled stereo imaging configurations and the overlapping region of measurement.

The mathematical model generated from the images of the calibration target allow for an exact overlap of the interrogation regions and corresponding two component vector measurements from each camera's image pair measurements. From these image pairs and the mathematical model, the two component velocity measurements made by each camera's images can be combining to derive the single true three component velocity vector representing the motion of the tracer particles in the flow field. The relationship the individual in-plane two component vectors and the out of plane component can be seen in Figure 2.6. 


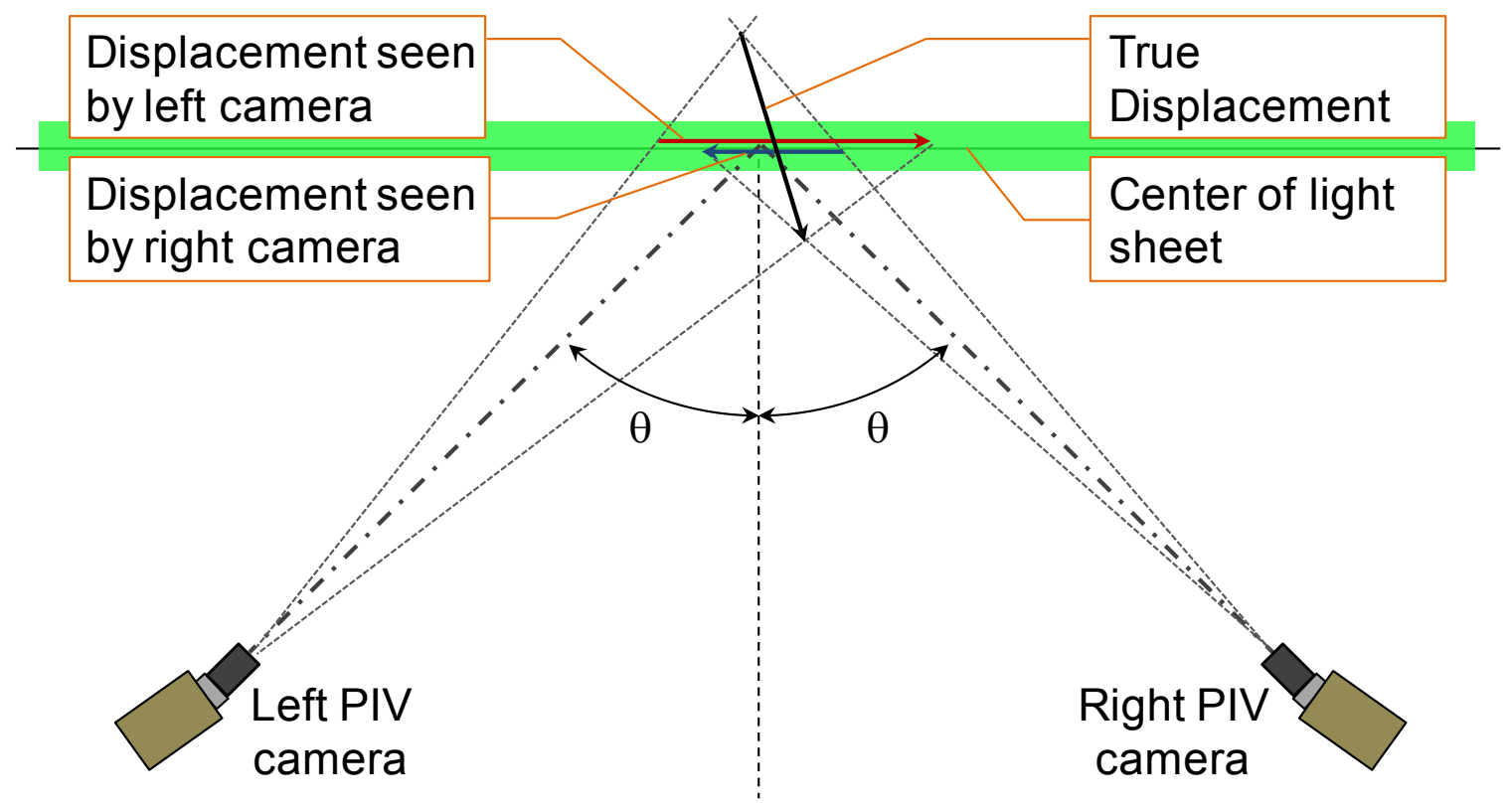

Figure 2.6 Measured velocity relation to true velocity for stereographic PIV measurement.

\subsection{Illumination}

\section{Laser Pulse Energy}

Selection of laser pulse energy is dependent on multiple factors including particle size, particle reflective index, camera angle, and test structure configuration. However, ideally, the laser pulse energy should be set as high as possible to maximize signal strength. The pulse energy should be increased until the imaged particle's peak intensity is just below the saturation point. This allows for maximum signal strength without clipping the signal, which results in a distortion of the light distribution and which is typically assumed to be a Gaussian distribution in the correlation algorithms and used for sub-pixel interpretation of the particle center point. 


\section{Alignment}

Proper alignment of the Nd:YAG dual cavity laser is the most critical step in obtaining PIV results. If the two laser cavities and beams are not properly aligned or exhibit significantly different beam profiles, coherent PIV results are nearly impossible to obtain. The Nd:YAG laser manual supplied by New Wave Research has a comprehensive alignment procedure that should be followed meticulously. To aid in laser beam alignment, burn paper such as ZAP-IT Laser Alignment Paper is recommended. The alignment paper also aids in checking beam intensity profiles, and detecting a clipped beam due to improper mirror placement in the laser head. The table below summarizes common issues that result due to improper laser alignment and placement in the camera's field of view.

Table 2.2

Laser alignment trouble shooting guidelines.

\begin{tabular}{|l|l|}
\hline \multicolumn{1}{|c|}{ Observed Issue } & \multicolumn{1}{c|}{ Laser Alignment Error } \\
\hline $\begin{array}{l}\text { Particle image intensity gradient } \\
\text { across field of view }\end{array}$ & $\begin{array}{l}\text { Light sheet is not centered on field of view, } \\
\text { results due to elliptical profile of light sheet } \\
\text { cross section }\end{array}$ \\
\hline Illuminated particle density gradient & $\begin{array}{l}\text { Light sheet is not centered on field of view, } \\
\text { results due to elliptical profile of light sheet } \\
\text { cross section, or focal point of light sheet is not } \\
\text { centered on field of view }\end{array}$ \\
\hline $\begin{array}{l}\text { Unequal number of particles } \\
\text { illuminated in first and second }\end{array}$ & $\begin{array}{l}\text { Difference in beam profiles between lasers, } \\
\text { check beam profile with burn paper at laser } \\
\text { head aperture and at the entrance and exit of } \\
\text { the light sheet optic to locate root cause }\end{array}$ \\
\hline $\begin{array}{l}\text { Incoherent particle movement within } \\
\text { interrogation region }\end{array}$ & $\begin{array}{l}\text { Light sheets are not aligned, therefore the first } \\
\text { and second light sheet pulses are illuminating } \\
\text { different sets particles, not the same set at } \\
\text { different points in time }\end{array}$ \\
\hline
\end{tabular}




\subsection{Optics}

There are three primary means to choose from when delivering the laser light from the laser head to the light sheet forming optics. For continuous wave and high speed lasers who's power output is lower, fiber optic means of beam deliver is possible, allowing for a highly versatile configuration for beam delivery to the light sheet forming optics. For high energy pulse lasers, the power output is typically well beyond that capable of being transmitted by fiber optic means, rather the beam must be routed by a series of mirrors. Many commercial PIV systems come with a light guide arm. The light guide arms are multi-jointed assemblies consisting of mirror holding tubes with typically six degrees of freedom (3). A light guide arms can be advantageous in cases where testing requires multiple light sheet positions, as they allow for easy placement and movement of the laser beam while maintaining mirror alignment. However, light guide arms produce some limitation in reach and range of motion, requiring the laser head to be near the measurement setup. Another option is a free standing mirror system; independent mirrors provide the highest degree of freedom in beam routing, with few limitations. However, alignment of all the mirrors can be tedious. In addition, movement of the light sheet for a series of test can become much more time consuming compared to the easy movement of a light guide arm.

There are numerous options for formation of the light sheet. Some commercial systems provide self contained optical assemblies for light sheet formation that attach directly to a light guide arm. Otherwise, individual optics can be used to form a light sheet, and by varying the optics used and relative orientation, the desired sheet characteristics can be obtained. Three basic optical configurations are outlined bellow, with each configuration using a series of three optics.

The first light sheet configuration is formed by a series of three cylindrical lenses. The first lens, with a negative focal length, diverges the beam vertically spreading the laser beam in to a light sheet. The second cylindrical lens collimates the diverging beam to create a light sheet of fixed height. Spacing between the first and second lenses controls 
the final height of the light sheet. The third cylindrical lens is used to control the light sheet thickness. The focal point of the light sheet, its thinnest point, is determined primarily by the focal length of the third lens (3). A light sheet with constant width, as is the case with this optical setup, is beneficial for measurements made inside a structure as the light sheet dimensions can be easily fixed for entrance and exit of the structure without spreading and reflecting light off walls. However, this setup is also limited, as the size of the light sheet is limited by the length of the cylindrical lenses. A general diagram for an optical setup using three cylindrical lenses is shown in Figure 2.7.
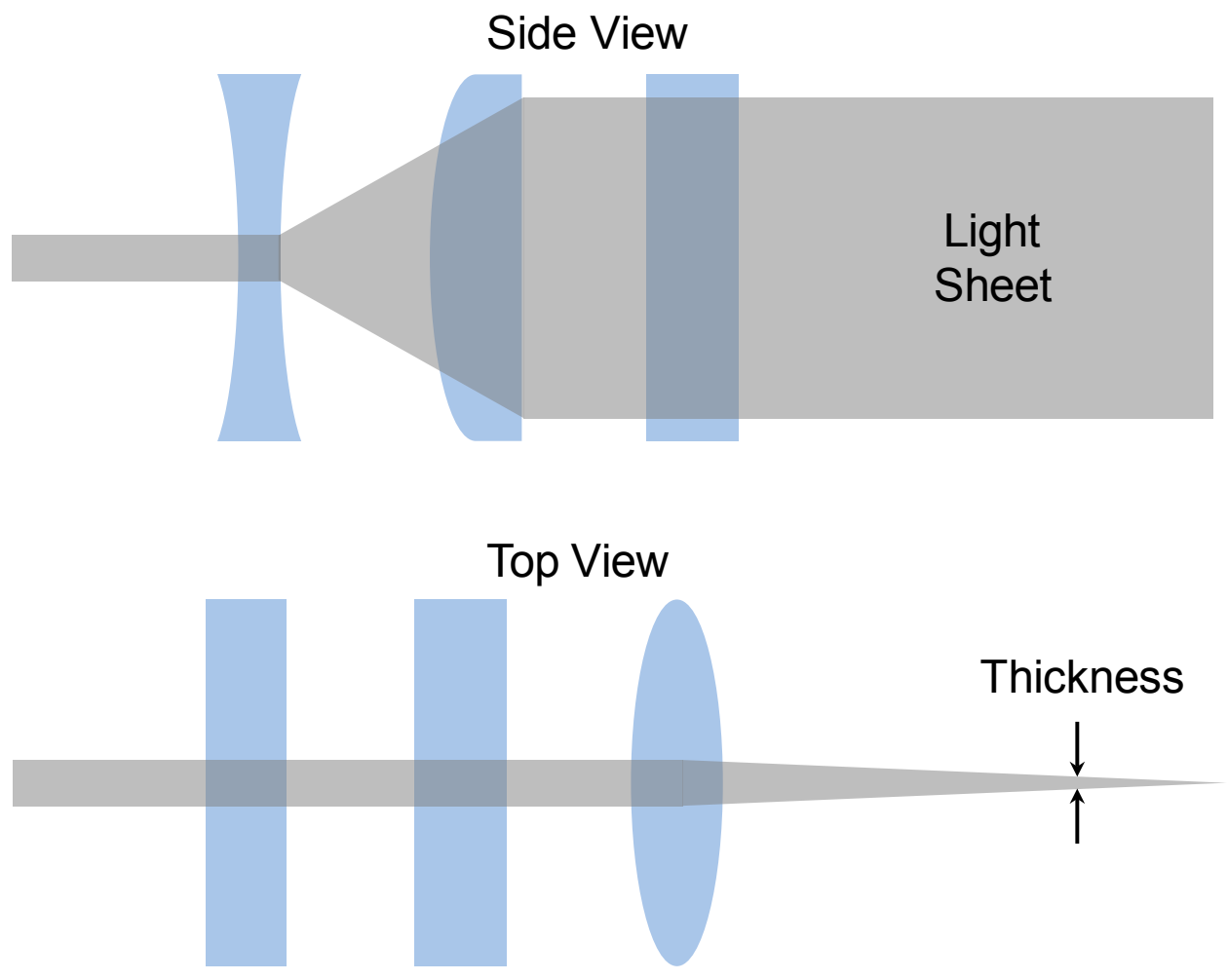

Figure 2.7 Non-diverging width light sheet created by passing a laser beam through a series of three cylindrical lenses. 
The second optical configuration for light sheet creation uses two spherical lenses and one cylindrical lens. The first lens is a spherical, negative focal length, lens used to diverge the beam vertically and horizontally. The second lens, a cylindrical lens, is used to control the rate at which the light sheet diverges vertically, while the spacing between the two spherical lenses dictate sheet thickness (3). Figure 2.8 depicts a typical layout for this optical configuration. The spherical-cylindrical-spherical lens configuration benefits from the ability to form a light sheet larger than the optics, an important factor for large scale PIV measurements. However, one drawback to this configuration is that the light sheet height and thickness are coupled and cannot be manipulated independently.

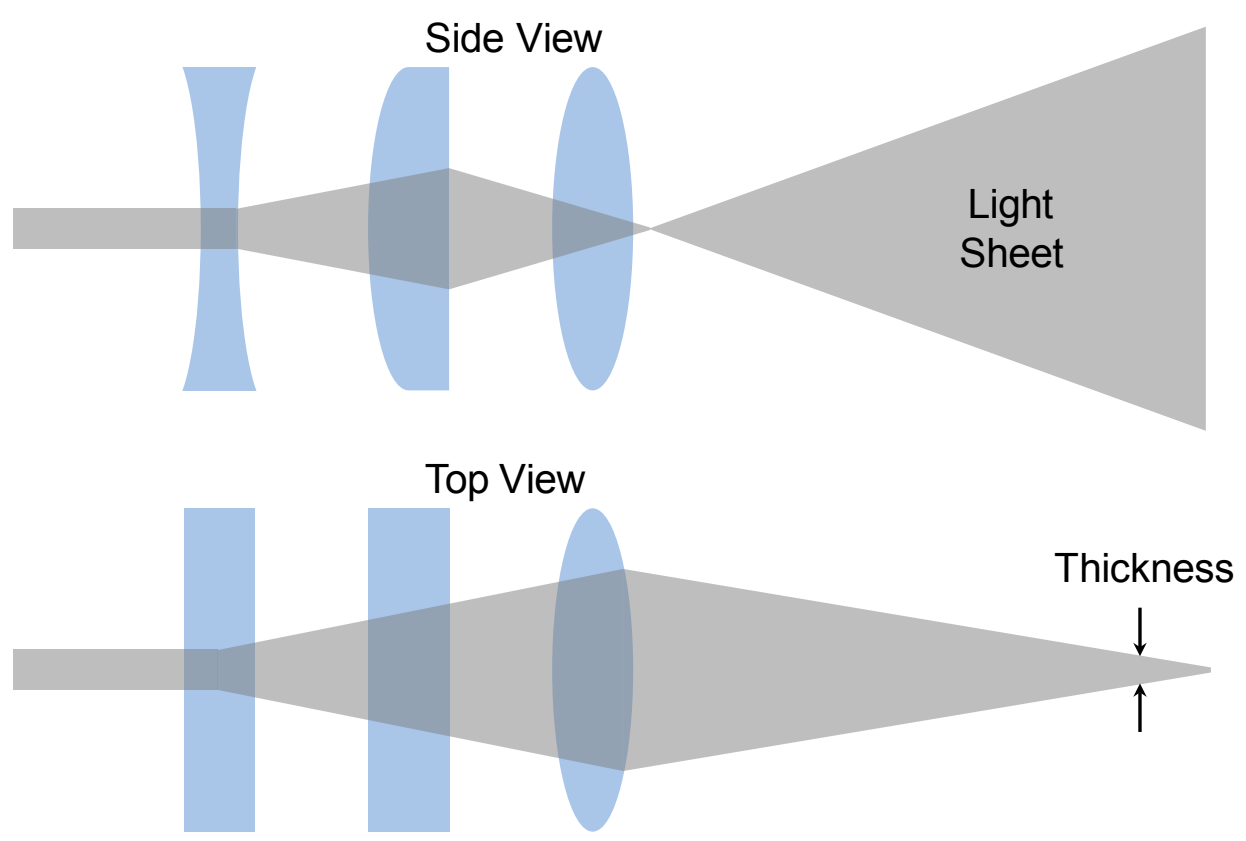

Figure 2.8 A diverging light sheet configuration using a spherical lens followed by a cylindrical lens and a final spherical lens.

The third optical setup for light sheet formation again consists of three cylindrical lenses as the first setup, but in a different orientation. Thin light sheets can be created using this configuration, useful for micro-PIV type applications where the desired light sheet 
thickness is less than the source beam diameter (3). The first cylindrical lens controls light sheet's vertical expansion rate by converges the laser beam vertically, with a short focal length falling before the second optic. This results in the light diverging prior to passing through the second optic. The second optic operates similar to the first, but is oriented $90^{\circ}$ offset to the first optic, resulting in horizontal convergence and divergence of the beam prior to the third optic. The cylindrical lens takes the rapidly diverging beam thickness and redirects it to create a thin, slowly converging light sheet as shown in Figure 2.9. This optical configuration benefits from independent control over sheet height and thickness. The thin nature of the sheet allows for the formation of a nominally high intensity light sheets, useful for gathering a strong light scattering signal from small particles.
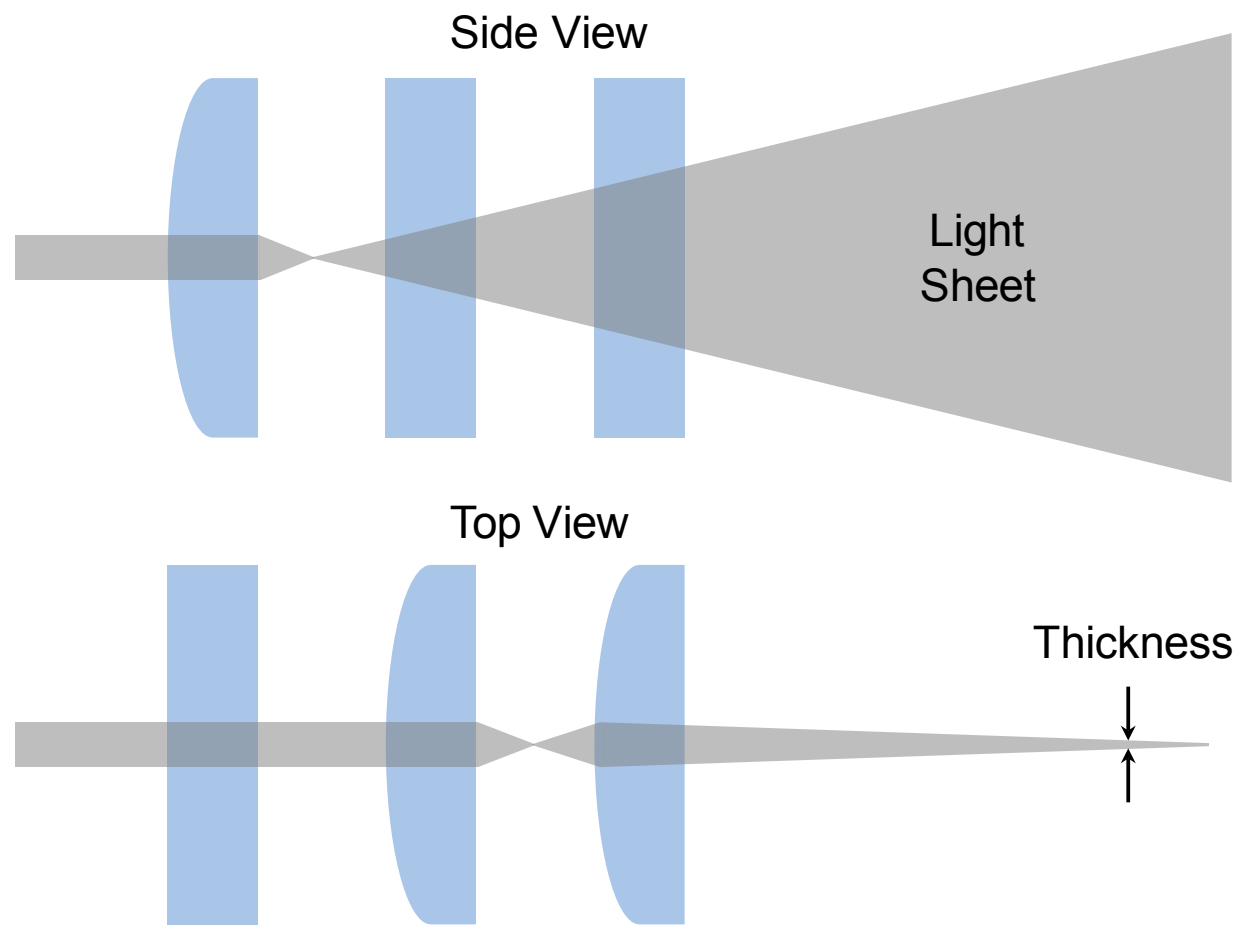

Figure 2.9 Three cylindrical lens configuration forming a thin diverging light sheet. 
The three optical configurations just described are but a few possible lens configurations that can be used to form a light sheet suitable for illumination of flows for PIV measurements. When configuring an optical assembly, it is important to remain couscous of certain considerations. First, the first optic to be placed in the beam path and last optic to be removed should be a lens that creates divergence in the laser beam. If a single lens is placed in the beam path, there is potential to operate the laser, focusing the beam to a point. With high energy pulse lasers this can create a hazard, as a focused beam has a tremendous energy density and can easily burn the coating on optics, particles in the air or even ignite materials. It is also important, when setting up a series of optics, to ensure that the focal point of a lens does not exist close to or within the following optic, for the same reasons as previously mentioned. Finally, all optics result in a degree of light reflection due to the change in refractive index at the surface. It is important to orient the lenses so that these reflections do not result in focal points on or in prior optics or possibly into the laser itself. Figure 2.10 shows both recommended and nonrecommended lens orientations and their corresponding light reflection paths. 

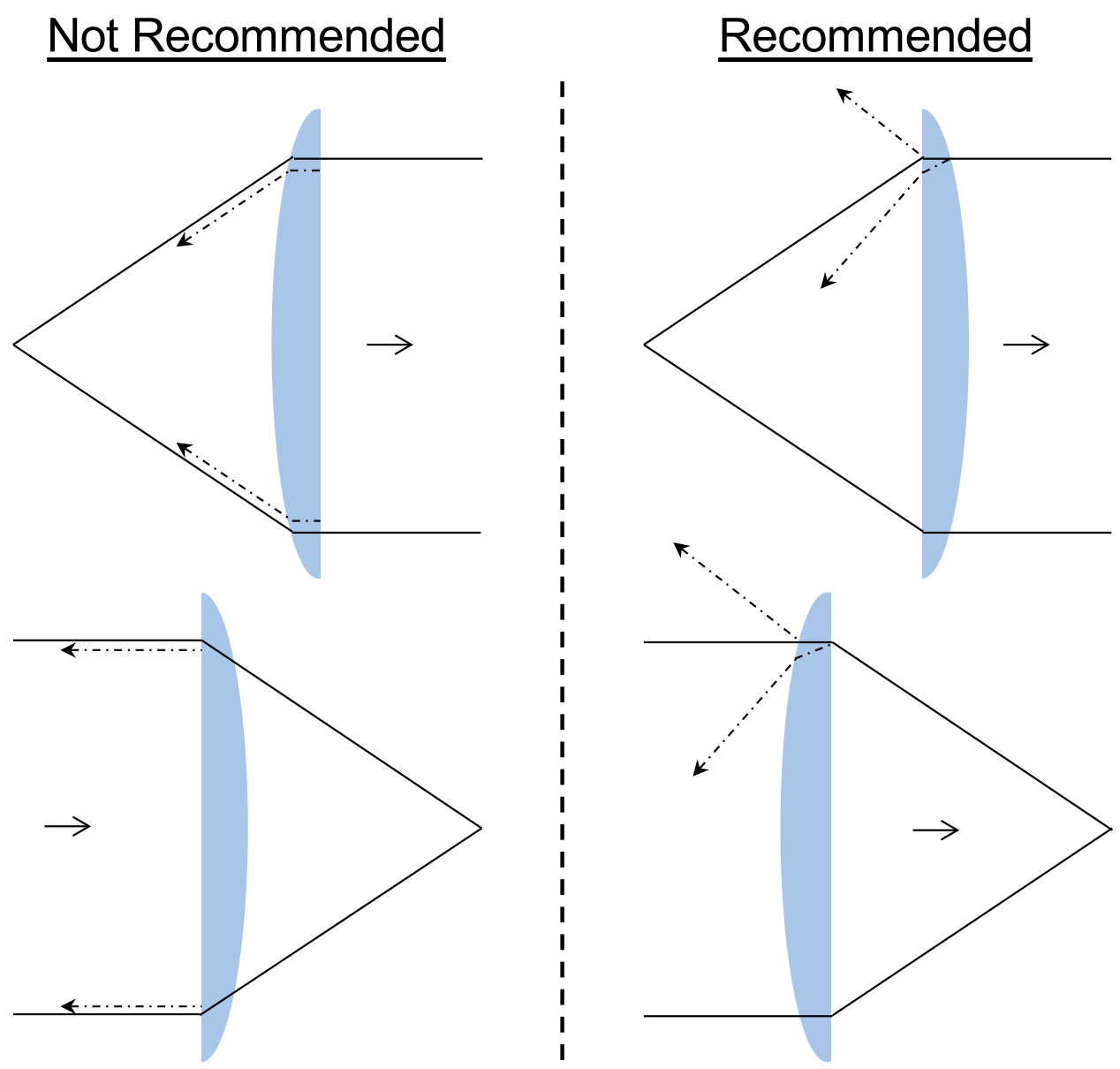

Figure 2.10 Refraction conditions due to optical orientation.

\subsection{Seeding}

There are several factors that must be taken into account to determine the seeding particle size required for a given flow. The seeding particle must be large enough that, for a given field of view and scale factor, the particles are represented on the camera CCD by multiple pixels to allow for sub-pixel calculations of particle positions. The seeding particle must also be large enough to scatter a sufficient amount of light for detection on the camera CCD; this is largely dependent on the amount of noise involved in a particular 
measurement case. Meanwhile, the seeding particle must be sufficiently small that it can accurately follow fluctuations in the fluid flow.

Stokes' Equations can be used to provide some an estimation of the ability of a particle to accurately follow its surrounding flow. In the case of air flows, where the particle density is substantially greater than the surrounding fluid density, the time response of a seeding particle's velocity can be estimated with the following two equations (3).

$$
U_{p}(t)=U\left[1-\exp \left(-\frac{t}{\tau_{s}}\right)\right]
$$

Where $\tau_{s}$ characterizes the relaxation time of a particle as a function the particle diameter and density as well as the dynamic viscosity of the surrounding fluid medium given by the equation:

$$
\tau_{s}=d_{p}^{2} \frac{\rho_{p}}{18 \mu}
$$

The seeding particles use in a PIV measurement should be capable of responding and equalizing with a change in flow equal to or less than the spatial resolution of the vector field being generated in order to accurately represent the fluid flow characteristics.

Seeding density is also an important parameter in obtaining good measurement results. The seeding density should be great enough that there are roughly fifteen or more particle pairs contained within the CCD pixel area covered by the desired interrogation region size to be used in the analysis of the raw PIV images. This allows for a strong correlation calculation and limits the influence of lost pairs, slightly unaligned light sheets, or other variations in a single measurement. At the same time, the spacing between particles should be greater than the displacement of the particles from the first to the second image frame. 
The ability of a particle to scatter laser light is a function of both the material's refractive index and geometric diameter. Therefore, a particle with a high refractive index and large geometric diameter, but low density is desired in most cases. In the case of liquid flows, where the required seeding particle size tends to be larger due to signal loss through the liquid before and after scattering from the particle, matching the density of the seeding particle with that of the fluid becomes more critical to avoid significant buoyancy effects.

Seeding particles can be grouped into two primary categories, liquid particles and solid particles, each with their own advantages and disadvantages in regard to both seeding particle properties and seeding implementation.

Liquid seeding particles can vary from atomized oil droplets on the sub micrometer scale to helium bubbles several millimeters in diameter.

Table 2.3

General characteristics of liquid and solid seeding particles.

\begin{tabular}{|c|l|l|}
\hline $\begin{array}{c}\text { Liquid Seeding } \\
\text { Particles }\end{array}$ & \begin{tabular}{l}
\multicolumn{1}{|c|}{ Advantages } \\
- easy clean up \\
- consistent nominal particle \\
diameter
\end{tabular} & $\begin{array}{l}\text { - degrade easily in harsh flow } \\
\text { environments } \\
\text { - less customizable for case by } \\
\text { case applications }\end{array}$ \\
\hline $\begin{array}{c}\text { Solid Seeding } \\
\text { Particles }\end{array}$ & $\begin{array}{l}\text { - don't evaporate over time } \\
\text { - can survive harsh } \\
\text { environments } \\
\text { - highly versatile to achieve } \\
\text { specific properties }\end{array}$ & $\begin{array}{l}\text { - prone to clumping from } \\
\text { moisture } \\
\text { - require stringent methods of } \\
\text { containment/capture from } \\
\text { escaping into lab environment }\end{array}$ \\
\hline
\end{tabular}


Table 2.4

General characteristics of solid and liquid particle seeders.

\begin{tabular}{|c|l|l|}
\hline $\begin{array}{c}\text { Liquid Particle } \\
\text { Seeding }\end{array}$ & \begin{tabular}{l}
\multicolumn{1}{|c|}{ Advantages } \\
- easy to achieve homogeneous \\
seeding \\
- easy to vary seeding rate \\
- consistent seeding rate
\end{tabular} & $\begin{array}{l}\text { - Only works for liquid } \\
\text { particles }\end{array}$ \\
\hline $\begin{array}{c}\text { Solid Particle } \\
\text { Seeding }\end{array}$ & $\begin{array}{l}\text { - highly controllable particle size } \\
\text { - can apply coatings to improve } \\
\text { refractive index } \\
\text { - control over particle density }\end{array}$ & $\begin{array}{l}\text { - irregular seeding rate } \\
\text { - difficult to alter seeding } \\
\text { density for a given seeder } \\
\text { - clumping issues due to } \\
\text { moisture }\end{array}$ \\
\hline
\end{tabular}

\subsection{Image Pre Processing}

\section{Thresholding}

The easiest and quickest means of simple noise reduction is by means of thresholding, where a fixed pixel intensity value is subtracted from the entire PIV image. This means of noise reduction is effective, but requires a large signal to noise intensity ratio to allow for noise elimination while avoiding signal elimination.

\section{Image Masking}

Image masking is the most effective means to prevent the PIV analysis of raw images from generating vectors where there is now seeded flow in the camera's field of view. This technique is applied directly in the FlowManager software. A more advanced form of image masking can be applied in MatLab to mask flow structure features using Matlab commands such as edge detection and blob analysis to give a more precise outline to the test structure than the freehand technique in FlowManager. 


\section{Constant Background}

In cases with a constant background profile, such as a slightly illuminated surface profile, or rotating machinery that is synchronized with the PIV imaging acquisition, the most effective means of elimination is by taking several background images without seeding, where the average of the background images is subtracted from the individual seeded PIV images. This technique does not work well when the laser is very near a surface resulting in high background image intensity specifically due to illumination by the laser due to variation in laser intensity pulse to pulse.

\section{Variable Background}

In the case of images containing varying backgrounds such as the case of rotating parts non-synchronous to the PIV imaging, or in the case of combustion applications where the constantly changing luminescence of the flame introduces a varying background noise, a more advanced approach is required. In this case, pre-processing of the raw image pairs in MatLab is required using image processing algorithms to detect, separate, and eliminate background profiles. The following is an example of a basic Matlab background detection and removal code:

cd ('C: Documents and Settings \Administrator \Desktop');

Imgl=imread('Test Image0000_b.tif'); \% Reads image into MatLab

Img1=double $(\operatorname{Img} 1) ; \quad \quad \%$ Converts 12 bit image into double format for processing

$\mathrm{SE}=$ strel('ball',9,9); \% Creation of a ball geometry structure element with $9 \mathrm{x} 9$ cell size

$\mathrm{FIR}=$ ones(9); $\quad$ \%Application of a FIR filter

$\mathrm{C}=1 . /$ filter2(FIR,ones( $\operatorname{size}(\operatorname{Img} 1))$ );

$\mathrm{BG}=$ imerode $(\operatorname{Img} 1, \mathrm{SE}) ; \quad \%$ Image background erosion

$\mathrm{BG}=\mathrm{C} . *$ filter2(FIR,BG);

Img1_processed=Img1-(BG); $\quad \%$ Subtraction of background from original image

The results of applying this code to a single image can be seen in Figure 2.11. Both images have the same intensity scaling applied. 

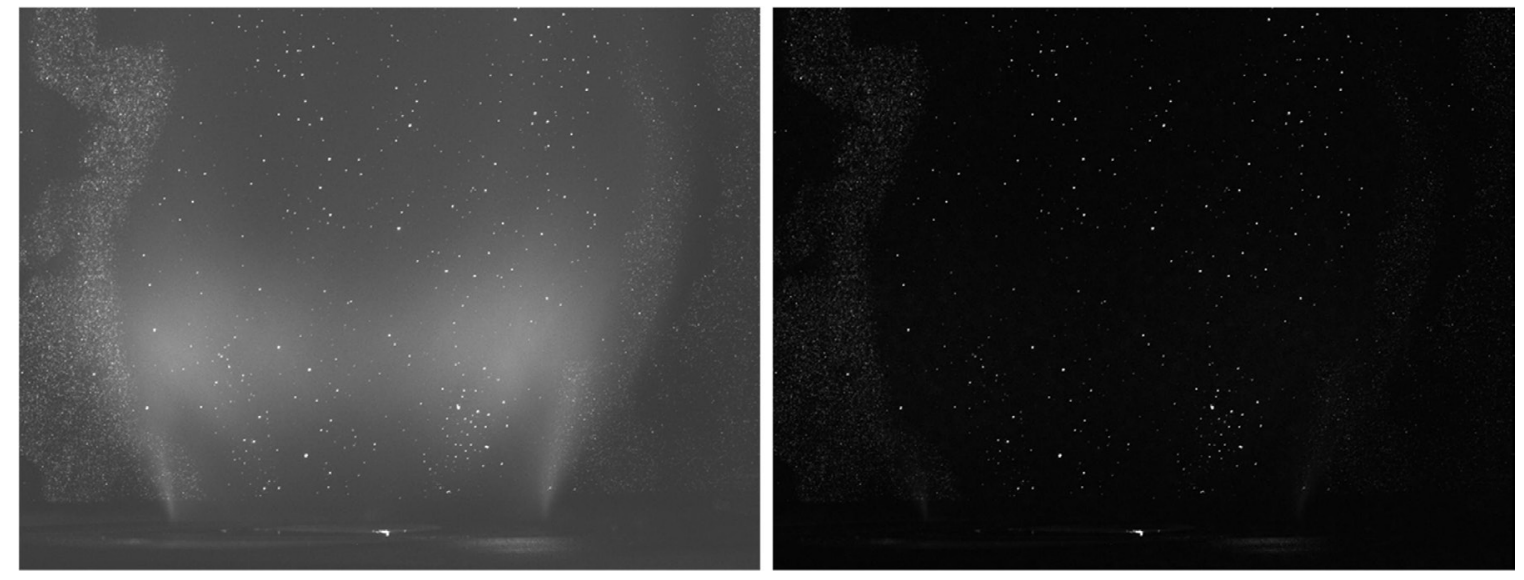

Figure 2.11 Matlab Background detection example (Left) raw PIV image (Right) PIV image with background removed.

This form of noise reduction is very powerful, but must be used with caution. The appropriate parameters to be used in the MatLab code may vary from application to application. It is also important to check the output vector maps to verify that the processing does indeed result in a more accurate representation of the flow structure.

\subsection{Vector Map Post Processing}

\section{Matlab Post-Processing of Vector Maps}

Matlab can be used as an effective tool for manipulation and presentation of both two and three component vector maps, but primarily the latter. Due to the limited options for presenting results in the FlowManager 3D-PIV work environment, basic Matlab code was developed for manipulation of exported three component PIV data files. Matlab can be used to manipulate the spatial scales in cases where the calibration plate axis intersection is arbitrary and a more relevant axis point is desired, i.e. the dump plane and swirler axis in the MGTC application at MTU. Matlab can also be used to separate out the individual components of the flow, display the flow on a three dimensional axis, add stream lines, present standard deviation and correlation coefficient profiles for the flow, and more. For 
two component velocity profiles, Matlab also enables the presentation velocity maps with strong velocity gradients easier to interpret by allowing the vector arrows to be of uniform length with a colored background showing the magnitude of the flow.

\subsection{Test Design Considerations}

\section{Interior Light Control}

Laser light access to the interior of the test article should be specifically limited to the flow region of interest captured in whole or part by the camera's field of view. The laser access window therefore, should be designed just large enough to allow illumination of this region. Likewise, if possible a laser light exit window should also be implemented for the light sheet to exit the test article. The exit window should be just large enough that the exiting beam does not hit the sides of the window or non-optical interior walls of the test article. This minimizes the stray light inside the test article, the amount of reflected laser light inside the test article, and the possibility of laser light reflecting off exterior objects and illuminating out of plane particles which will cause erroneous velocity measurements.

Dead ending of the laser light at an interior surface should be avoided if at all possible. This results in large amounts of reflected light inside the test article and extremely high light intensities near the wall washing out seeding particle signals near the surface and if the surface inside the camera's field of view potential damage to the CCD can occur burning out individual pixels.

If dead ending of the light sheet inside the test article is unavoidable, several layers of Flow Visualization Components' $532 \mathrm{~nm}$ fluorescent paint should be applied to the surface at and near the point of incidence. The rest of the interior surface should be coated with a non-reflective coating, such as flat black paint, if testing conditions permit to absorb laser light scattered by the seeding particles minimizing illumination of the background as well as out of plane seeding particles. 
Measurements planes running parallel and very near surfaces should be avoided, as the light scattering from particles near the surface illuminate the surface resulting in a high noise floor making it difficult to distinguish particles from the background when small seeding particles are used.

\section{Exterior Light Control}

Strict control over stray lighting in the lab as well as control over the laser light sheet outside of the test article should also be exercised to prevent light leaking into the camera lens reducing the signal to noise ratio in the images. To achieve this, the separation between the shroud mounted to the light guide optic arm base should be separated from the laser head just enough to allow for pulse energy level measurements during test setup. The laser light sheet between the exit of the light sheet optic and the light inlet window on the test article should be shrouded as well as from the light sheet exit window to a suitable beam dump. The interior of these shrouds should consist of a light absorbing material to minimize the propagation of reflections within the shroud, both flat black paint and black felt are effective at absorbing stray laser light. Theses shrouds not only minimize out of plane seeding illumination and light leakage into the camera but also aid in protecting the operator from stray laser reflections.

\section{Optical Window Selection}

There are multiple considerations when choosing a material for the laser light sheet inlet and exit windows as well as for optical access of the camera. The material should be resistant to degradation from the seeding medium, primarily chemically resistant in the case of liquid seeding and abrasion resistant in the case of solid seeding particles. The refractive index of the window should also be minimized to maximize the signal strength reaching the camera lens. In addition, the window materials must also withstand cleaning and structural requirements of the test article. In the case of PIV measurements, where a visible $532 \mathrm{~nm}$ laser light is used, there is a multitude of choices for window material selection. However, for PLIF measurements where ultraviolet $266 \mathrm{~nm}$ laser light is used and typical window materials such as Plexiglas or common silica glass are visibly 
transparent but have opaque properties in the ultra violet spectrum, the range of choices is significantly reduced to materials such as quartz and sapphire.

The optical windows should also be designed for easy removal and cleaning without disturbing the test article to allow for cleaning between tests. Although some seeding materials are much easier to clean than others, for example DiEthylHexyl Sebacate (DEHS) oil compared to olive oil - two common liquid seeding materials, all seeding particles will eventually build up on the windows, requiring cleaning between tests; especially for prolonged test periods or test conditions are being performed.

\subsection{Measurement Uncertainty}

Determining the uncertainty in a measurement is difficult when it comes to optic based measurements, as there are many factors that can influence the measurement, some of which can be removed or minimized and others that cannot. The PIV measurement technique is no exception, determining the measurement uncertainty for a single measurement is rather difficult.

Uncertainty in PIV measurements can be separated into two types of error, bias errors and random errors. Bias errors result from the limitations of the fundamental correlation processing basis of PIV measurements. Since these errors are systematic, they can potentially be reduced or eliminated through careful consideration of test setup parameters. Random error accounts for the other source of measurement uncertainty, and cannot be eliminated or reduced as easily as bias errors.

A practical means of determining the degree of uncertainty in a specific application of PIV is through performing a PIV measurement with the hardware and test setup configured to identically matching those under data collection with a controlled measurement field. This can be done by either taking measurements of a static particle field, or taking measurements in a flow field with known motion properties. Experimental estimations of measurement uncertainty are limited as they providing insight into total measurement uncertainty resulting from both bias and random error 
without insight into the individual contributors to uncertainty in a measurement. In contrast, numerical simulations can provide insight into how specific parameters in a PIV measurement contribute to overall measurement uncertainty.

Monte Carlo based numerical simulations, in contrast to experimental estimations, can provide insight into how individual aspects of a test can influence the uncertainty in a PIV measurement. The measurement errors studied in numerical simulations represent the bias errors corresponding to limitations in the base correlation analysis fundamental to PIV. By defining the intensity profile of a laser light sheet and the light scatter from a particle, typically both of which are commonly assumed to follow a Gaussian distribution, a simulated flow field and corresponding intensity profiles for images of the flow field can be simulated with complete control. Numerical modeling and control over particles characteristics, light sheet profiles, and particle motion, allows the study of a range of measurement characteristics and their influence on uncertainty. Key PIV measurement characteristics for uncertainty studies include particle size, particle displacement between exposures, particle image density, lost pairs, digital image quantization levels, background noise influence, displacement gradients in interrogation regions, and the influence of out of plane motion. There is an extensive range of parameters as well as the relationships between parameters and their influence on measurement uncertainty which could be studied and discussed each in extensive detail. However, some of the broader insights from these studies are easily implemented in practice to reduce the bias errors associated with PIV measurements.

Uncertainty studies have shown that for cross-correlation based measurements a particle image diameter of 2-2.5 pixels is optimum. Since correlation processors commonly use three discrete points (pixel intensity values) to estimate the Gaussian light scatter profile of a particle, image diameters smaller than two pixels tend to introduce a pixel-locking condition in which particle displacements are bias to integer pixel values, large particle image diameters results in the three points representing a small portion of the Gaussian profile resulting in increased error in peak estimation (3). 
Particle displacement studies have shown that the displacement uncertainty is substantially reduced when particle displacement drops below half a pixel. This characteristic in displacement uncertainty can be exploited in practice through the implementation of offset interrogation regions as used by adaptive correlation based analysis methods (3). Particle image density, characterized by $N_{I} F_{i} F_{o}$ as previously discussed, is critical to the probability of valid peak detection while taking into account the loss of both in plane and out of plane image pairs. While greater quantization in the image sensor provides a more accurate measure of the discrete intensity measurements used for light scatter profile and peak estimation, studies have found that there is minimal difference in peak estimation error for quantization above four bits (3). The same studies have shown that background noise below ten percent has minimal influence on measurement error, as error in the correlation limitations dominate until background noise becomes in excess for quantization levels above four bits (3). Since an interrogation region is represented by a single vector, gradients in particle displacement within the measurement region inherently lead to error. However, for a given displacement gradient, the associated error in measurement can be minimized through use of small interrogation regions. By using a small interrogation region, the difference between actual displacements of particles in the measurement region and the calculated average displacement is minimized, reducing the error in measurement. 


\section{CHAPTER 3 : DANTEC DYANMICS SYSTEM}

\subsection{Dantec Dynamics Hardware Overview}

\section{System Hub}

The Flow Manager System hub allows communication and synchronization among the user interface computer and measurement hardware. The System Hub consists of several key components: the laser control board, two frame grabber boards, the LIFO buffer memory, data synchronization board, onboard CPU, and PC interface as shown in Figure 3.1. The laser control board sends TTL pulses to the two laser power supplies to trigger both flashlamp and Q-switch events for both illumination pulses. The frame grabber boards receive the digital images taken by the PIV camera(s) which are then transferred to the PC memory and storage or to the HUB's onboard LIFO memory buffer for later upload to the PC. The PC interface allows for easy user control and setup of the HUB acquisition parameters through a GUI. The synchronization board controls the TTL trigger signals sent to the camera(s) for image acquisition, which can be triggered independently based on user input, or based on one of four independent trigger pulse inputs. The HUB is also capable of monitoring and recording up to three channels of analog data for easy synchronization with the PIV measurement events.

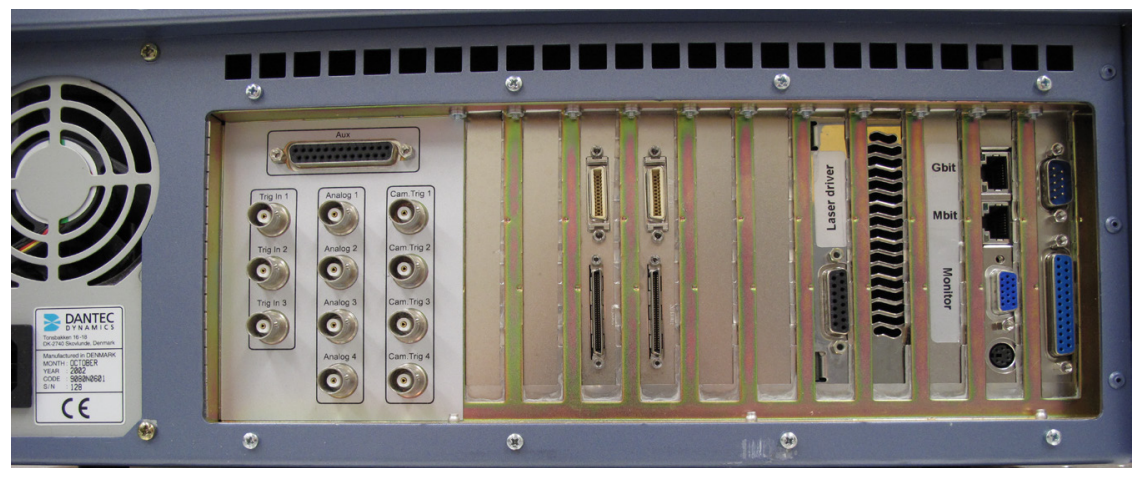

Figure 3.1 Dantec Dynamics FlowMap system hub. 


\section{Laser}

The laser used by the Dantec Dynamics PIV system is Electro Scientific Industries' (ESI) New Wave Research Gemini PIV 200 dual cavity Nd:YAG pulse laser. The specifications for this laser are shown in Table 3.1 and the primary components of the laser head are shown in Figure 3.2.

Table 3.1

Laser specifications for New Wave Research's Gemini PIV 200 pulse laser (8).

\begin{tabular}{|l|c|}
\hline Pulse energy $(532 \mathrm{~nm}) /$ head & $200 \mathrm{~mJ} @ 15 \mathrm{~Hz}$ \\
\hline Pulse energy $(266 \mathrm{~nm}) /$ head & $120 \mathrm{~mJ} @ 15 \mathrm{~Hz}$ \\
\hline Beam diameter & $\sim 5.5 \mathrm{~mm}$ \\
\hline Energy stability & $\pm 5 \%$ \\
\hline Pulse width & $3-5 \mathrm{~ns}$ \\
\hline Beam divergence & $<2 \mathrm{mrad}$ \\
\hline Beam pointing & $<200 \mathrm{urad}$ \\
\hline Jitter & $\pm 0.5 \mathrm{~ns}$ \\
\hline
\end{tabular}

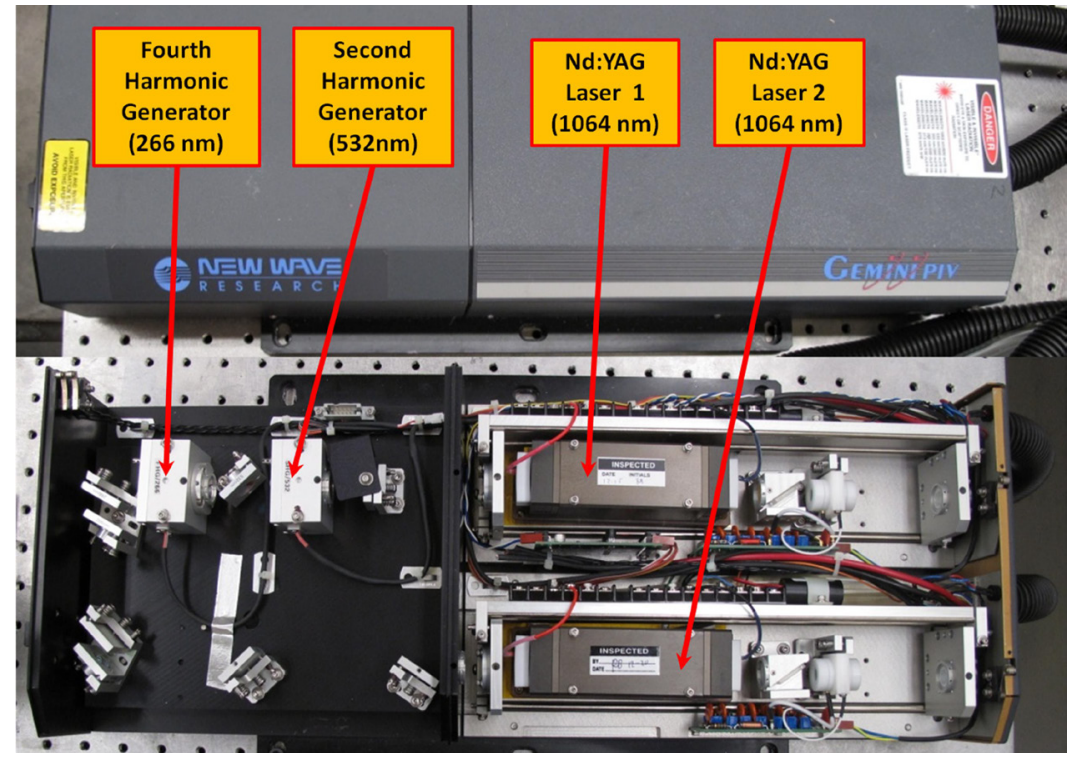

Figure 3.2 New Wave Research Gemini 20015 Hz Nd:YAG laser. 


\section{Cameras}

The HiSenseMkII PIV cameras used in the Dantec Dynamics PIV system are manufactured by Hamamatsu C8484-05c model cameras, specifically designed for dualframe PIV imaging. The camera's CCD sensor is designed with an optimum sensitivity to $532 \mathrm{~nm}$ light, matching the second harmonic output of Nd:YAG lasers (9). Camera specifications relevant to PIV imaging are outlined in Table 3.2. Unique to cameras designed for PIV, the first frame exposure time is much shorter than the second exposure time, 4.5 micro seconds and 88496 micro seconds respectively. The first exposure is required to be significantly shorter to avoid the frame being exposed to the second illumination pulse which can be separated from the first pulse by mere micro seconds when imaging high speed flow cases. The second exposure is much longer, as the data must be transferred off the camera onto the system hub memory buffer rather than to a second set of cells as in the case of the first frame exposure.

Table 3.2

Specifications for Hamamatsu's C8484-05C PIV camera (9).

\begin{tabular}{|l|c|}
\hline Sensor Resolution & 1.37 MegaPixels \\
\hline Sensor Array Dimensions & 6.45 um by $6.45 \mathrm{um}$ \\
\hline Pixel Dimensions & $8.67 \mathrm{~mm}$ by $6.60 \mathrm{~mm}$ \\
\hline Sensor Size & 12 bit \\
\hline Analog-Digital Resolution & $1700: 1$ \\
\hline Dynamic Range & $5.6 \mathrm{~Hz}$ \\
\hline Double Frame Acquisition Rate & 4.5 us (frame 1$), 88496$ us (frame 2) \\
\hline Double Frame Exposure Times & \\
\hline
\end{tabular}

\section{Stereo mounts}

For stereo PIV, Dantec Dynamics stereo mounts and baffles shown in Figure 3.3 were used. These mounts facilitate easy setup of the PIV cameras in the required scheimpflug 


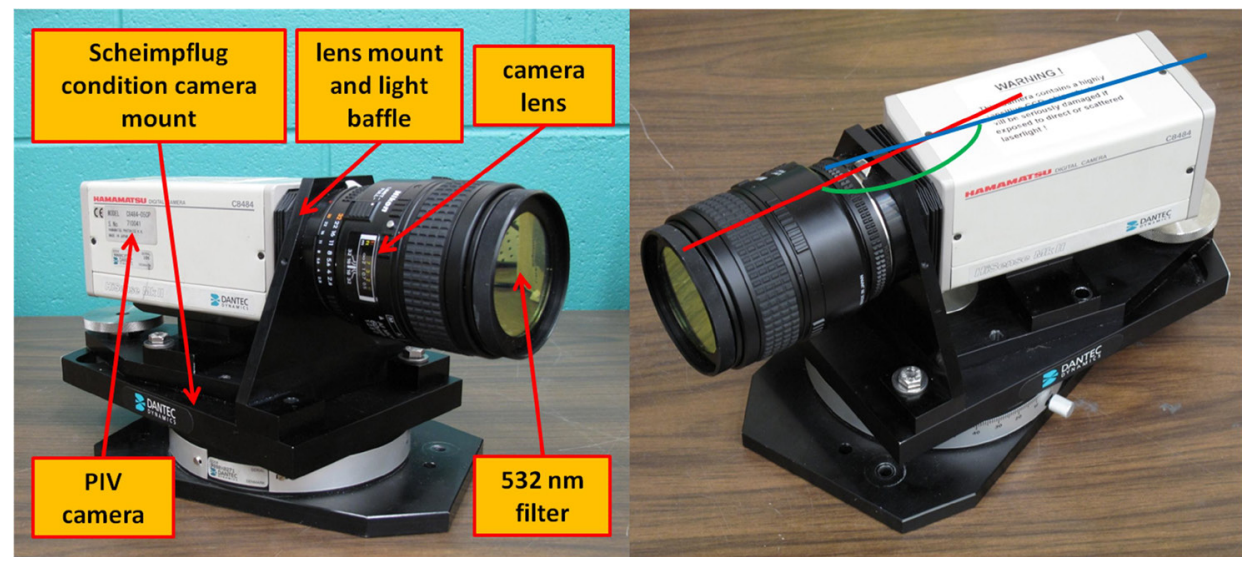

Figure 3.3 Stereo PIV camera assembly (left) depiction of offset between lens and senor plane satisfying Scheimplug condition (right).

orientation, offsetting the camera sensor and lens planes to allow alignment of the measurement plane and focal plane of the imaging system.

\section{Lens}

The lenses used in the PIV system are Nikon's AF-Micro-NIKKOR 60mm f/2.8D lens. These lenses provide a smaller field of view than typical camera lenses, enabling a high spatial resolution for PIV measurement purposes. Specifications for the Nikon lenses are documented in Table 3.3. Dantec Dynamic's PIV lens filters, model 80C41, are designed for peak transmission at $532 \mathrm{~nm}$ with a +/- $10 \mathrm{~nm}$ cutoff.

Table 3.3

Nikon AF Micro-NIKKOR lens specifications (10).

\begin{tabular}{|l|c|}
\hline Focal Length & $60 \mathrm{~mm}$ \\
\hline Aperture Range & $\mathrm{f} / 2.8-\mathrm{f} / 32$ \\
\hline Angle of View & $26^{\circ} 30^{\prime}-39^{\circ} 40^{\prime}$ \\
\hline Lens Elements & 8 \\
\hline Lens Groups & 7 \\
\hline Filter Size & $62 \mathrm{~mm}$ \\
\hline
\end{tabular}




\section{Light Guide Arm}

Dantec's PIV system also includes both a 1 meter light guide arm as well as an attachable light sheet optic assembly for adjustable sheet thickness with a divergence of approximately $\pm 14^{\circ}$ both of which are shown in Figure 3.4. The counterweighted light guide arm provides easy movement of the light sheet during testing, while maintaining beam alignment.

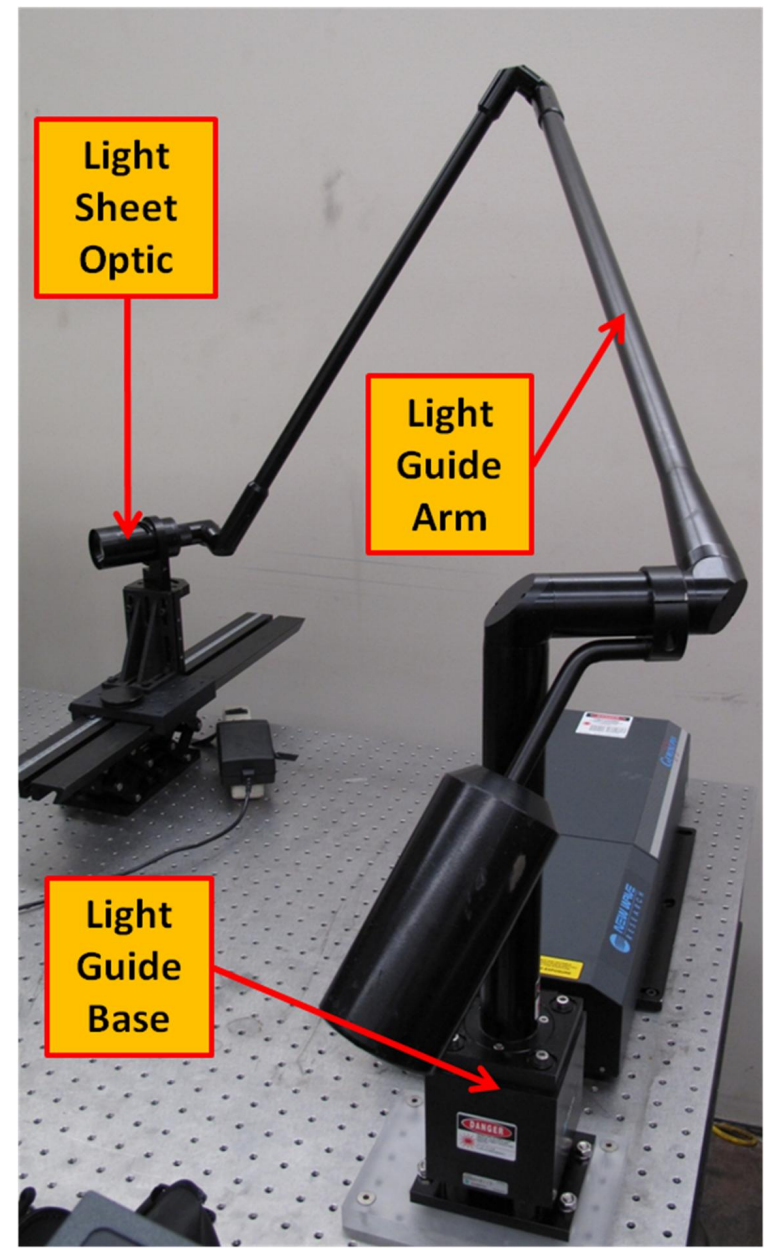

Figure 3.4 Dantec Dyanmic's light guide and sheet optic assembly. 


\section{CHAPTER 4 : APPLICATIONS}

\subsection{Testing Overview}

This chapter covers three laboratory applications of particle image velocimetry system. Each test having a specific motivation, application, PIV configuration, and test conditions. As a whole the applications to which the PIV system was applied were chosen based on upon multiple objectives:

1. The experimental applications were chosen to provide experience in applying particle image velocimetry techniques to a range of flow conditions in order to utilize and explore the range in capabilities of the PIV system.

2. The PIV included both 2-D and 3-D stereographic PIV measurement configurations.

3. Complex test apparatus have been explored for applications of PIV under conditions which prove challenging for measurements to be made.

4. Applications were chosen in which the measurement results are of interest and benefit to the CSEL and AERB general areas of research.

From these considerations, three experimental applications were carried out. The first experiment involved applying the 2-D PIV measurement technique to a flow channel with tumble strips installed, providing a near wall measurement application, where a good signal to noise ratio can be difficult to obtain due to reflections of the laser light sheet. The second experiment utilized the PIV system's 3-D stereographic measurement capabilities in the measurement of a strong three dimensional swirled flow, as well as being a challenging environment for imaging due to noise induced by the flame luminosity in a reacting model gas turbine combustor. The reacting flow also provided a measurement environment containing significant gradients in all three components of the velocity field. The third application was to a fan-induced turbulent flow in a closed combustion chamber. The physical constraints of the test subject limited PIV 
measurements to two dimensions, a challenge in both measurement execution and measurement comprehension due to the strong three dimensional flow characteristics.

\subsection{PIV Measurements in a Flow Channel}

\section{Objective}

This testing had three primary objectives. The first objective was to setup a flow field with large scale flow characteristics to serve as an intitial application of the PIV equipment in the lab and make a successful PIV measurement. Second, was to make a successful measurement near a boundary surface, a task known to be difficult for PIV application due to light reflections. The third objective was to implement the use of a fluorescent paint as well as various noise reduction techniques to improve signal quality in the measurement.

\section{Motivation}

The first application of the particle image velocimetry system was to the tumble strip lined flow channel provided by the project sponsor, Pratt \& Whitney. The testing was broken into three components. In the first test, the horizontal measurement plane was positioned mid-stream in the flow channel with the camera's field of view capturing flow above the $6^{\text {th }}$ and $7^{\text {th }}$ pair of tumble strips. For the second test, the same measurement region was used, but a bluff body was inserted into the channel directly upstream of the measurement region splitting the flow in two. This test was designed as an easy application for initial implementation of the 2-D PIV measurement system. The bluff body was intended to introduce large scale flow structures within the PIV measurement region, while a mid-stream measurement plane was implemented to avoid complication with taking PIV measurements near boundary surfaces. For the third test, the bluff body was removed from the channel and the measurement plane was moved down between the two sets of tumble strips. This test was desirable for two reasons. First, Pratt \& Whitney had performed prior thermal measurements on the tumble surface to which they wanted to correlate velocity measurements. Second, the tumble strip measurement provided an 
application of 2-D PIV measurements near a contoured boundary surface. PIV measurements near surfaces are typically difficult for multiple reasons. The first obstacle to overcome is if the light sheet is incident to the surface at a point near the measurement region. The incident light can reflect into the camera and either wash out the particle signal making flow measurements impossible, or if the reflection off the surface is strong enough and focused, burn out the camera's sensor resulting in permanent damage to the PIV system. Even if the light sheet is parallel to the boundary surface, this can still cause measurement issues in multiple ways. The close proximity of the light sheet to the boundary surface can illuminate the surface in the camera image, creating bright spots and shadows in the image background, which reduce the image's signal to noise ratio making proper correlation difficult and typically requiring some type of image processing to the raw image prior to applying the particle tracking algorithms. The other issue can be the reflection of light off the boundary surface and illumination of out of plane particles in the flow. This leads to faulty results in the correlation process between the first and second image frames.

\section{Hardware Setup}

For all tests, two primary hardware components were used to create the test flow. First was a large air plenum with dimension of $45.7 \mathrm{~cm} \times 45.7 \mathrm{~cm} \times 53.3 \mathrm{~cm}$. The air enters the plenum from the side with the tumble strip channel attached to an adjacent side wall of the plenum. The building's compressed air source was used to supply a consistent flow to the test section using an in-line pressure regulator to meter the flow. A schematic of the test setup is shown in Figure 4.1. 


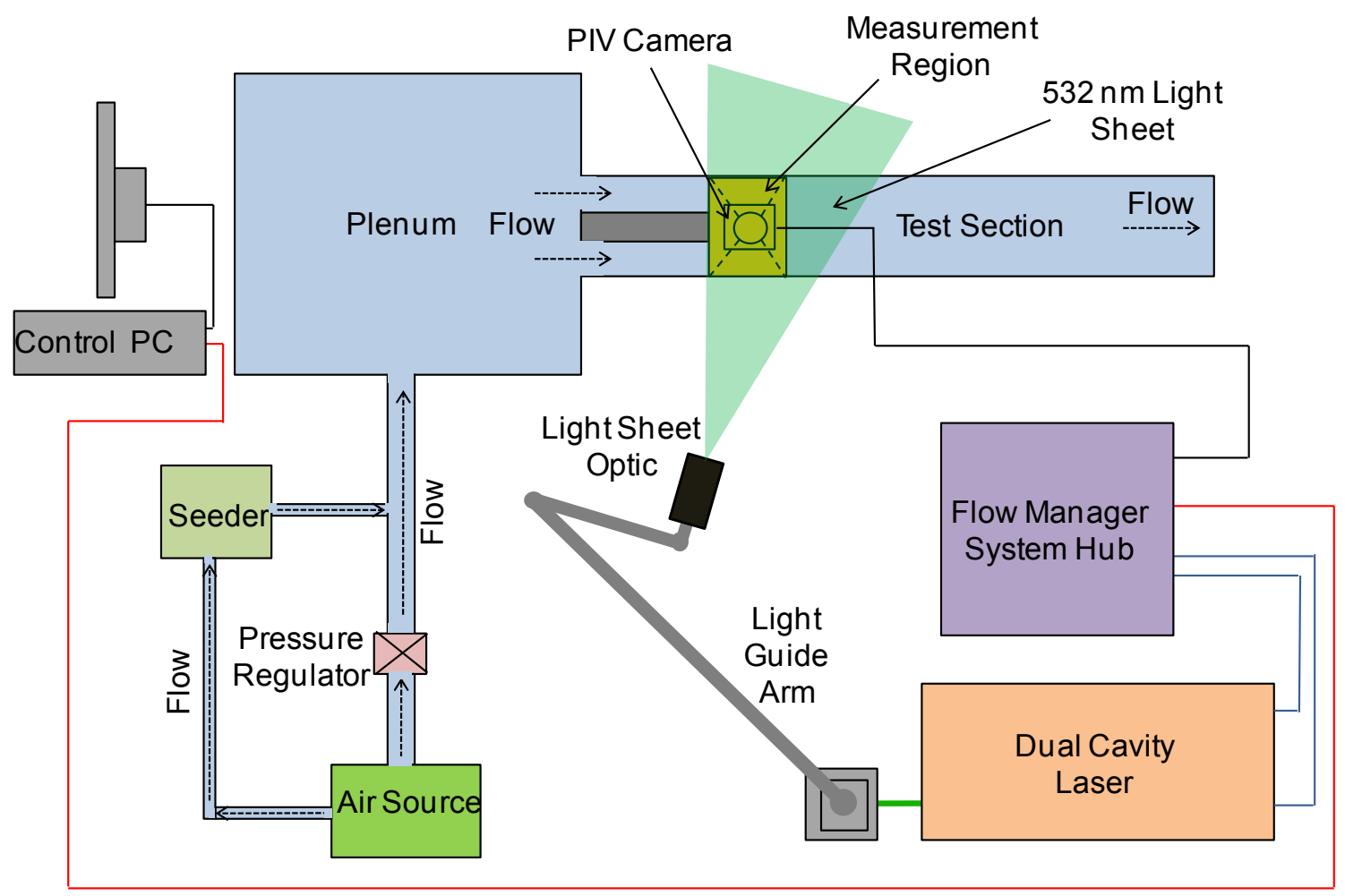

Figure 4.1 Schematic of test hardware and PIV component setup in tumble strip flow testing (with bluff body installed).

The tumble strip channel attached to the plenum measures $82.6 \mathrm{~cm}$ long with a channel width of $9.1 \mathrm{~cm}$ and height of $5.3 \mathrm{~cm}$. Attached to the bottom surface of the channel were 19 pairs of equally spaced tumble strips, each with a height of $0.51 \mathrm{~cm}$ and $5.1 \mathrm{~cm}$ long. A model of the tumble strip channel is shown in Figure 4.2.

For the second test, using a bluff body to split the channel flow, a thin sheet was laid over the tumble strips creating a smooth walled flow channel with a $9.1 \mathrm{~cm}$ by $4.8 \mathrm{~cm}$ cross section. The bluff body, measuring $19.1 \mathrm{~cm}$ long, $2.5 \mathrm{~cm}$ wide, and $4.8 \mathrm{~cm}$ tall was then inserted into the flow channel with the end of the bluff body located at the upstream boarder of the camera's field of view in the measurement region. Dimensions of the test section, bluff body, and measurement region are shown in Figure 4.3 


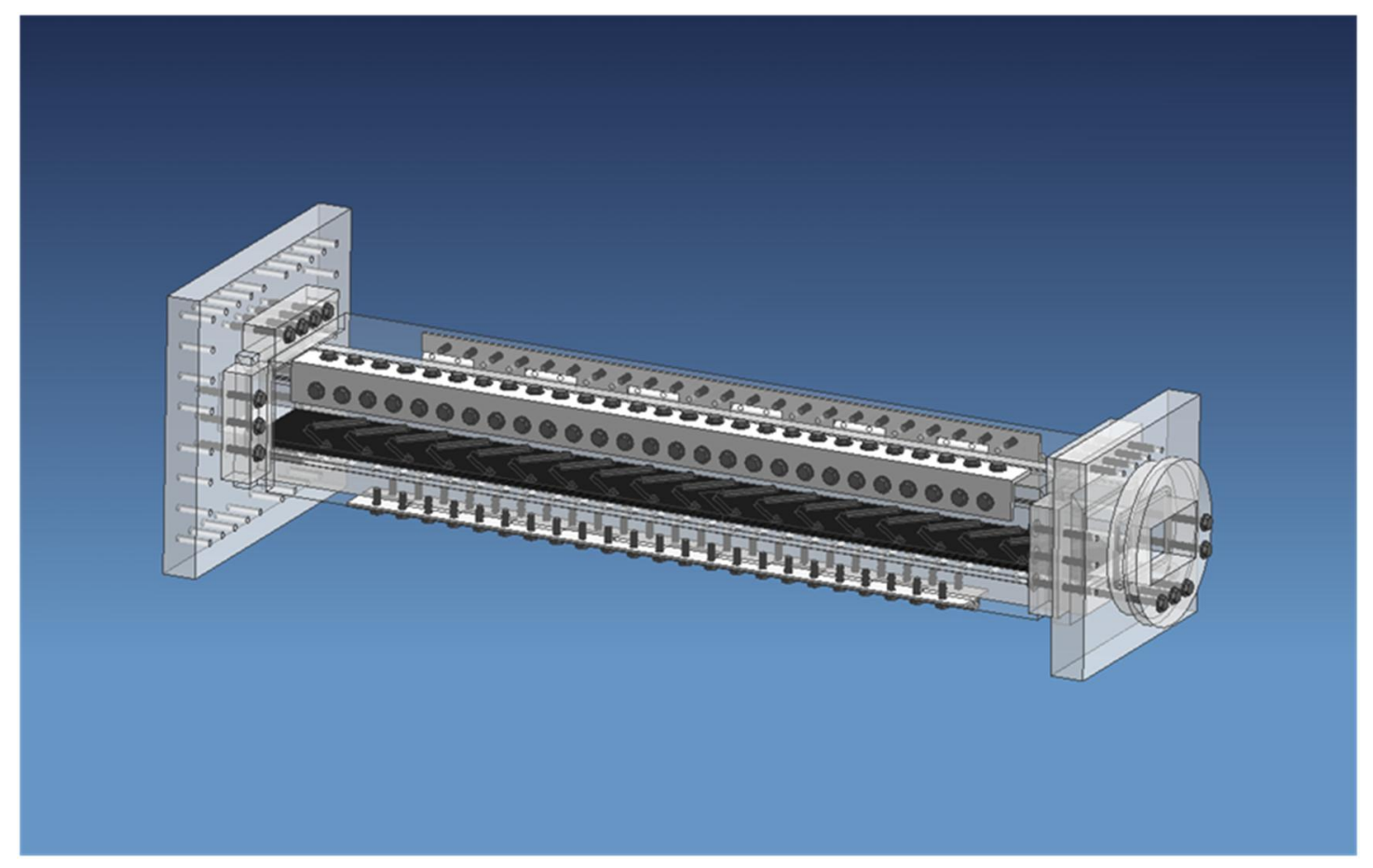

Figure 4.2 Tumble strip channel model.

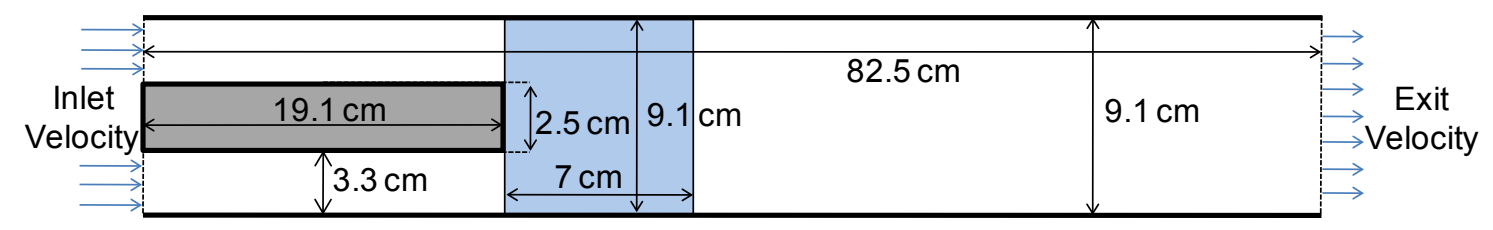

Figure 4.3 Tumble strip flow channel dimensions with bluff body (grey) and PIV camera FOV (blue). 


\section{PIV Setup}

For this testing, a two component planar PIV setup was used. The light sheet was oriented horizontally and generated using Dantec Dynamics' light guide arm and optic assembly. The light sheet was formed with a $0.5 \mathrm{~mm}$ nominal thickness in the measurement plane for the first two tests and a $1 \mathrm{~mm}$ nominal thickness for the inter tumble strip flow test. The PIV camera was positioned above the flow channel to capture the flow region spanning the width of the channel from the start of the $6^{\text {th }}$ pair of tumble strips to near the end of the $7^{\text {th }}$ pair of tumble strips as shown in Figure 4.4. The same camera field of view was used for all three tests.

Initially, tracer particles in the form of DEHS oil particles with a 1 micrometer nominal diameter were provided by the Dantec Dyanmics aerosol seeding system. However, the Dantec system, with its 8 to 120 seeding nozzle operation produced excessive seeding for

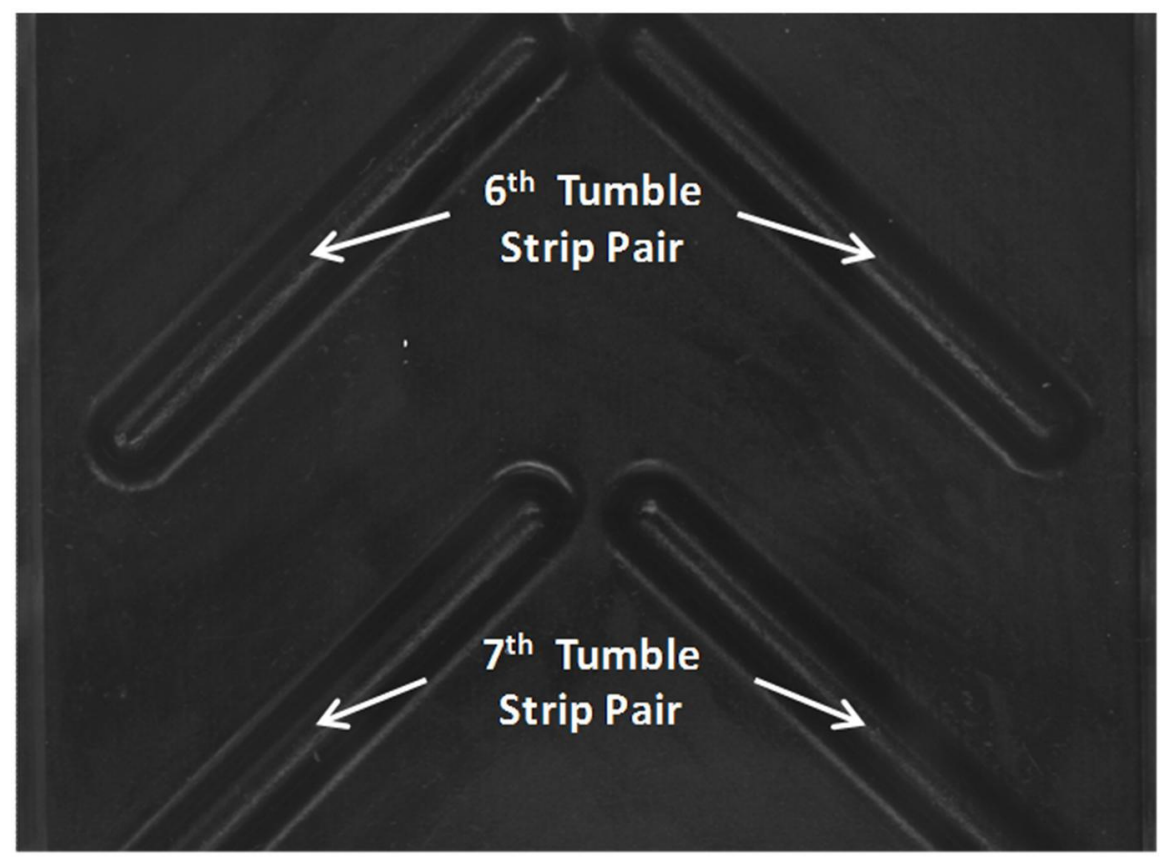

Figure 4.4 Background image and reference for the PIV camera's field of view. 
the air flow rates in this study. To overcome this issue, a single nozzle seeder was fabricated, supplying a lower seeding rate of DEHS oil particles suitable to the air flow rates being used. A schematic of the seeder design can be seen in Figure 4.5. A small portion of the air flow was diverted from the compressed air source upstream of the pressure regulator and plumbed into the single nozzle seeder, the seeded output air form the atomizer was then injected back into the primary air flow prior to the mixture entering the air plenum. This setup allowed for mixing to occur prior to the flow entering the flow channel, resulting in a homogenously seeded flow at the measurement plane as required for quality PIV measurements.

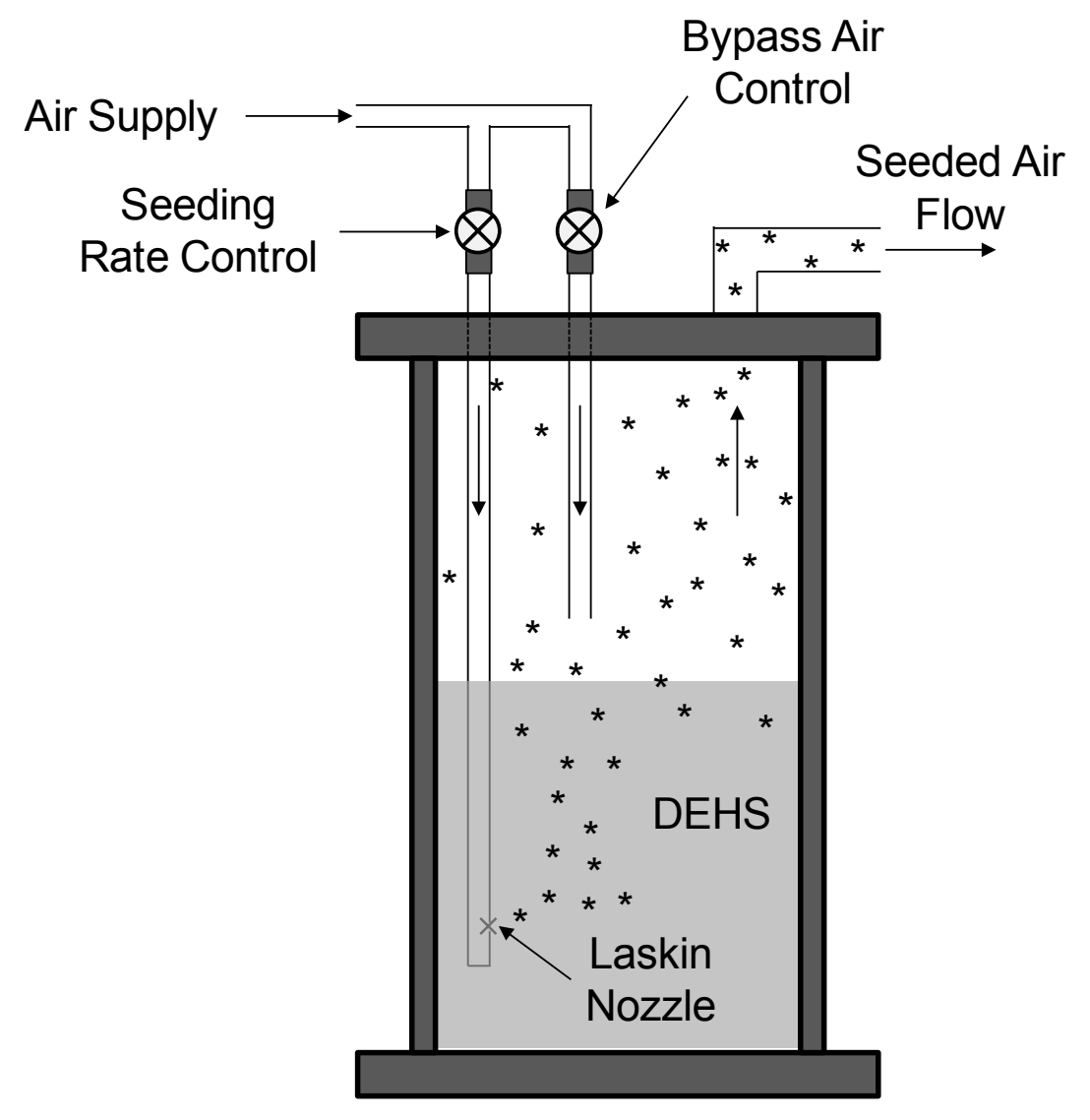

Figure 4.5 Schematic of a single nozzle aerosol seeding system. 


\section{Test Conditions}

Flow around the bluff body was measured under two flow conditions. For the first test air flow was regulated to produce a flow velocity of $0.8 \mathrm{~m} / \mathrm{s}$ at the center of the flow near the exit of the channel. The light sheet, centered in the flow channel was adjusted to a sheet thickness of $0.5 \mathrm{~mm}$ with a pulse energy of $75 \mathrm{~mJ}$ per pulse. A series of 150 images were taken to generate an average measurement of the flow. The time interval between measurements in this case was 450 microseconds.

The flow around the bluff body was again measured as in the first test, but with the air supply regulated to produce a flow velocity of $2.0 \mathrm{~m} / \mathrm{s}$ under the same measurement conditions. The light sheet thickness, pulse energy, and number of measurements taken were unchanged. However, the time interval between measurements was shortened to 200 microseconds to account for the higher flow velocities in this case.

For both flow conditions, the bluff body flow images were processed using an adaptive correlation process. A final interrogation region size of $32 \times 32$ pixels was used with a three step correlation process, central differencing interrogation region offset, and a relative peak validation of 1.2

In addition to moving the measurement plane down to $2.5 \mathrm{~mm}$ off the bottom surface of the flow channel, centering the laser sheet on the tumble strips, the light sheet thickness was increased to $1 \mathrm{~mm}$. The light sheet thickness was increased to account for the greater out of plane component in the flow as the air rises and falls across the tumble strips. For this test, the air flow rate was returned to $0.8 \mathrm{~m} / \mathrm{s}$. To minimize reflections several means of light control were implemented. First, the $6^{\text {th }}$ and $7^{\text {th }}$ tumble strips were coated with a fluorescent paint, which absorbs the $532 \mathrm{~nm}$ laser light and emits in the $560 \mathrm{~nm}$ light spectrum, outside of the PIV lens filter cutoff range. Second, physical light barriers were placed between the sheet forming optics and the flow channel, allowing only a narrow light beam to enter then flow channel and illuminate the space between the $6^{\text {th }}$ and $7^{\text {th }}$ tumble strips. A third beam block was used between the camera and the measurement plane to block reflections off $6^{\text {th }}$ tumble strip opposite of the light sheet. 


\section{Results and Discussion}

It was found that the single nozzle seeding system's range in particle generation rate was suitable to the flow rates and allowed for fine adjustment of the seeding rate to achieve the desired seeding density required for PIV flow measurements. Figure 4.6 shows an image of the illuminated flow field downstream of the bluff body as well as the seeding density provided by the single nozzle seeding system captured by a color digital camera. The image also shows the homogeneous distribution of seeding particles throughout the flow, a critical characteristic of seeding for accurate PIV measurements.

In the case of the split flow using the bluff body, computational models were built for flow comparison under the two flow conditions. Simulation of the flow was performed using Ansys' Fluent computation fluid dynamics software. The simulation used the RNG $\mathrm{k}-\varepsilon$ turbulence model with enhanced wall treatment to model the two dimensional flows. The modeling used a finite volume approach, implementing the SIMPLE algorithm and using a first order upwind scheme for flow. Convergence was checked using a continuity error less than $1 \times 10^{\wedge} 6$. For the $0.8 \mathrm{~m} / \mathrm{s}$ flow, the simulation converged on a solution, shown in Figure 4.7 and Figure 4.8.

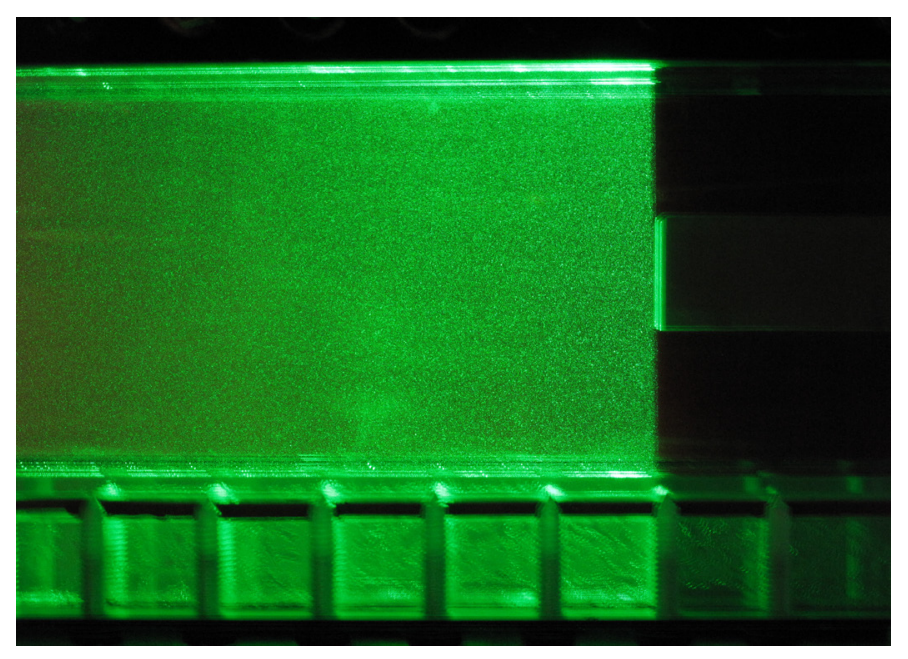

Figure 4.6 Image capturing laser illumination of channel flow downstream of the bluff body. 
The vector profiles for simulation and experimental measurement shown in Figure 4.7 indicate fairly good agreement as also shown in Figure 4.8, showing contour plots of the flow field. In the experimental case, flow is slightly biased to the left side of the bluff body, corresponding to the side of the air plenum from which the compressed air enters. The small region of low velocity positioned between the left side primary flow and low velocity region in the wake of the bluff body corresponds to several damaged pixels on the camera's CCD sensor. In the case of the PIV measurements, the low velocity regions at the edges of the experimental measurement field are attributed to a loss of image pairs.

In the $2.0 \mathrm{~m} / \mathrm{s}$ exit velocity flow case, the PIV measurements showed flow formations and characteristics similar to those seen in the $0.8 \mathrm{~m} / \mathrm{s}$ case. However, with the greater flow velocity, larger gradients in the flow were experience in the bluff body wake depicted in Figure 4.9. The characteristics of the flow on each side of the bluff body remained the same, with the left flow experiencing slightly greater velocity due to orientation of the incoming air to the test section.

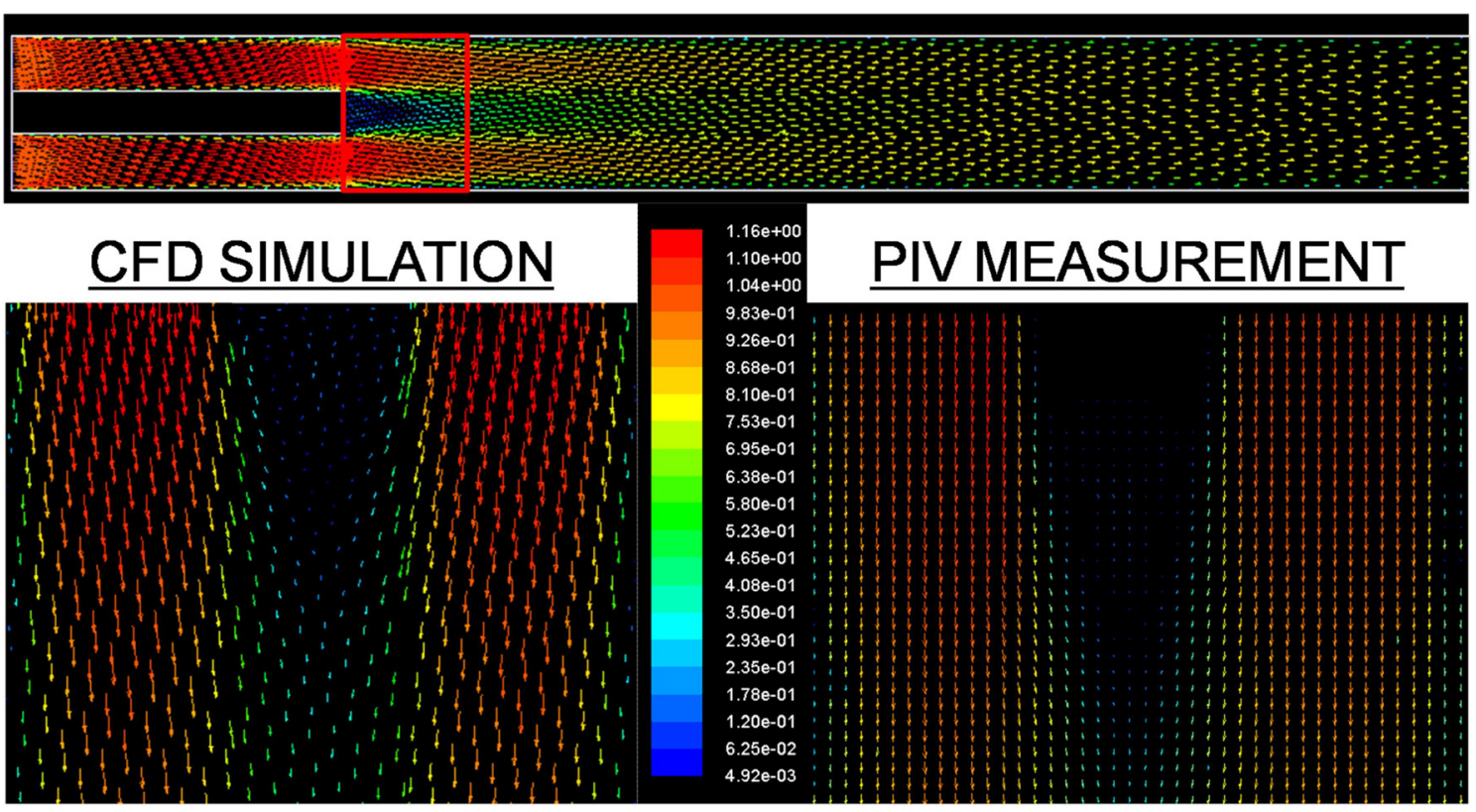

Figure 4.7 CFD and PIV comparison of bluff body flow at $0.8 \mathrm{~m} / \mathrm{s}$ exit velocity. 


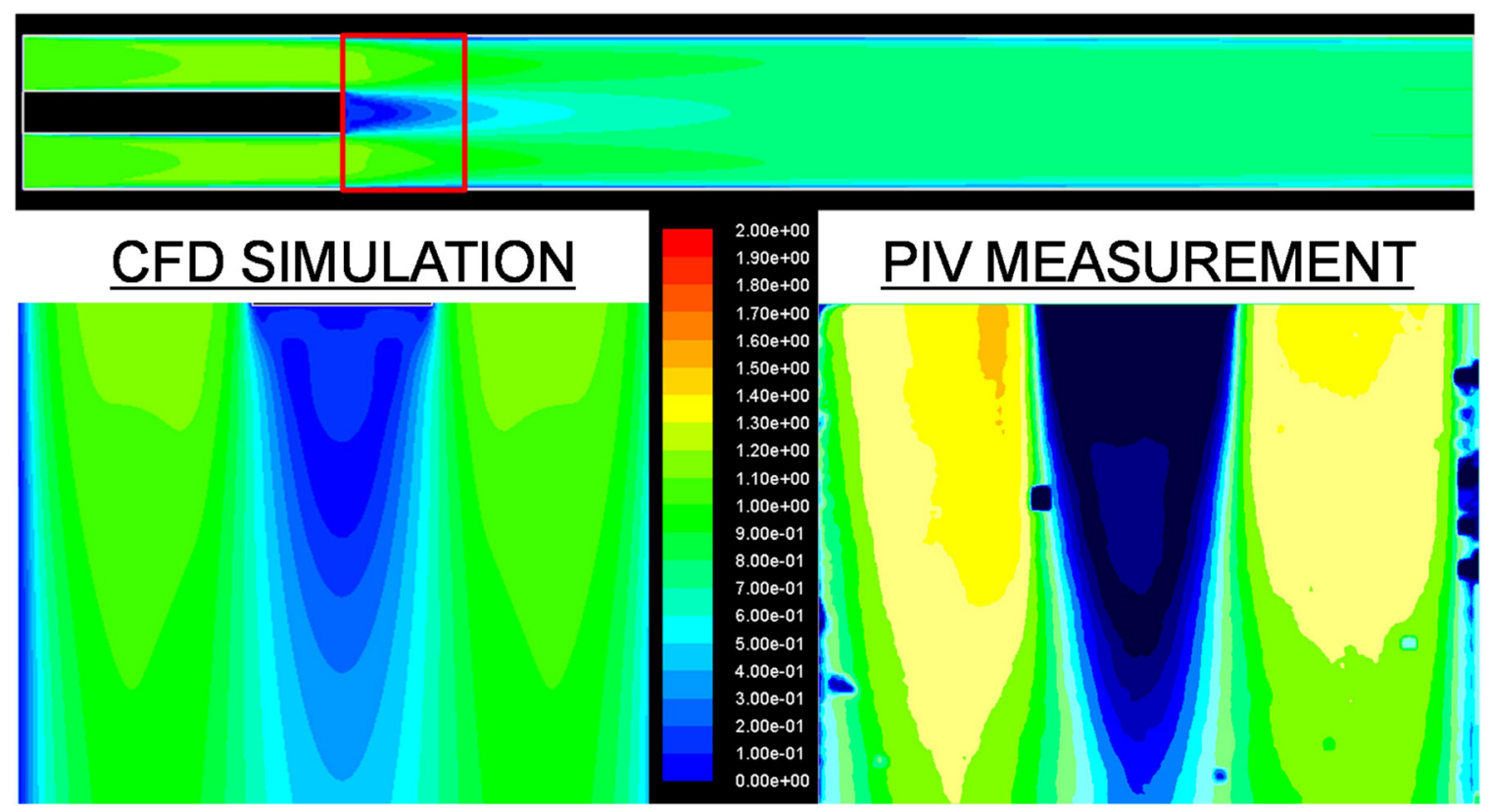

Figure 4.8 Comparison of numerical and experimental flow magnitude $(u+v)$ profile for $0.8 \mathrm{~m} / \mathrm{s}$ flow condition.

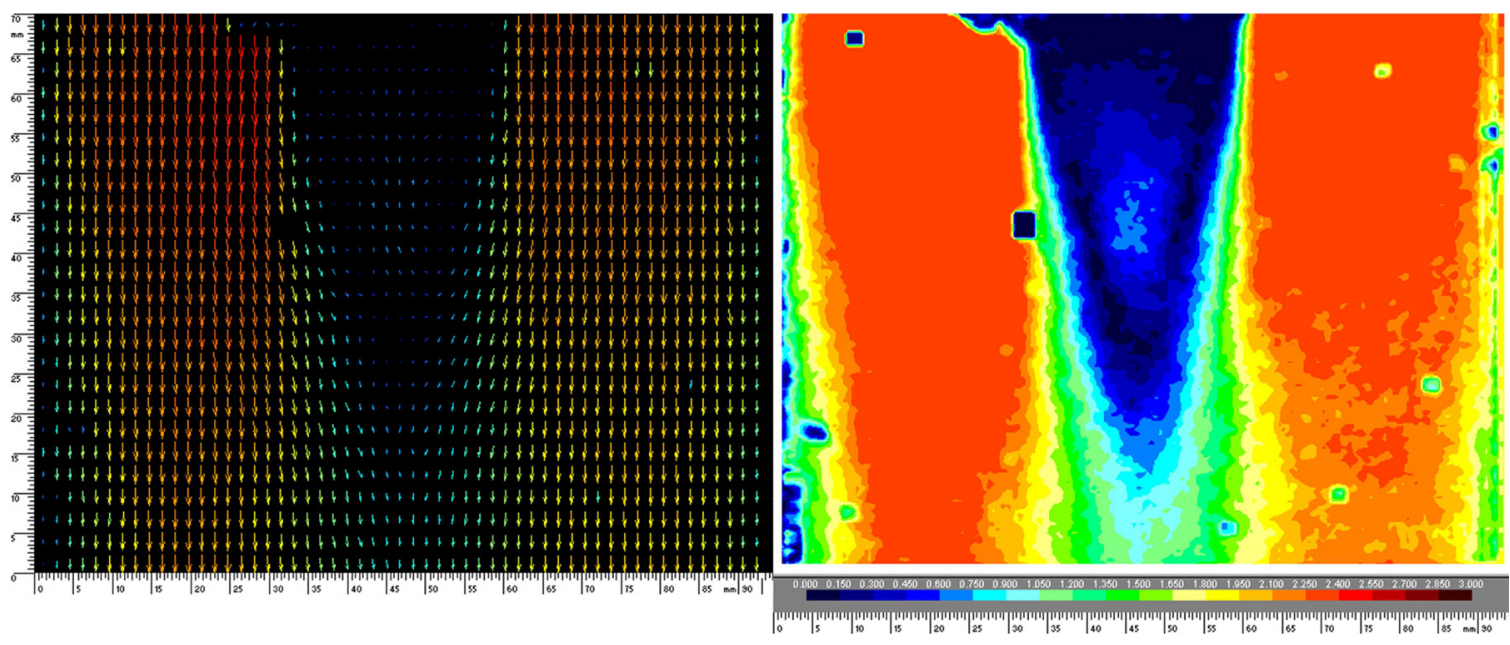

Figure 4.9 Vector profiles (left) and contour of flow magnitude (right) for PIV measurement of bluff body separated $2.0 \mathrm{~m} / \mathrm{s}$ flow case. 
The series of test have been conducted to measure the flow between tumble strips, with the light sheet only $2.5 \mathrm{~mm}$ above the channel floor allowed for measurements to be made. Figure 4.10, a photograph taken of the laser light sheet inside the flow channel, shows the limited light sheet. As the figure shows, the light sheet was physically limited in sheet width to approximately $3.8 \mathrm{~cm}$ with sheet forming optics aligned parallel to the tumble strips. Aligning the optics and tumble strip axis allowed for full illumination of the void between tumble strips, but also minimized the intensity of any reflections along length of the $6^{\text {th }}$ and $7^{\text {th }}$ tumble strips. Since the tumble strips run symmetrically down the flow channel, the desired measurement region was limited to the left half of the flow channel. This allowed for the high intensity reflection of the light sheet off the adjacent $6^{\text {th }}$ tumble strip on the right side of the flow to be blocked by placement of a barrier between the PIV camera and the flow channel. The fluorescent paint also aided in separating particle light scatter from surrounding reflections or illumination by the light sheet.

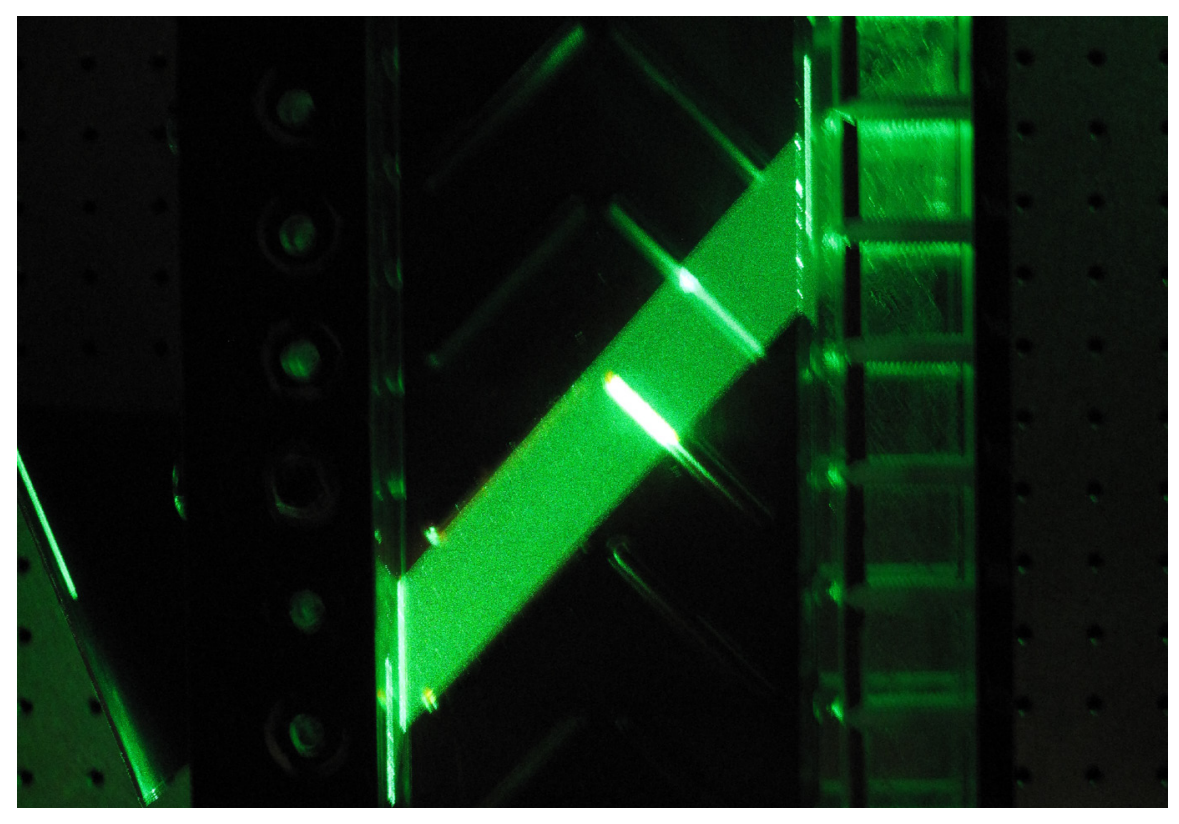

Figure 4.10 Limited illumination of flow channel for flow measurement between 6 th and 7 th tumble strip. 


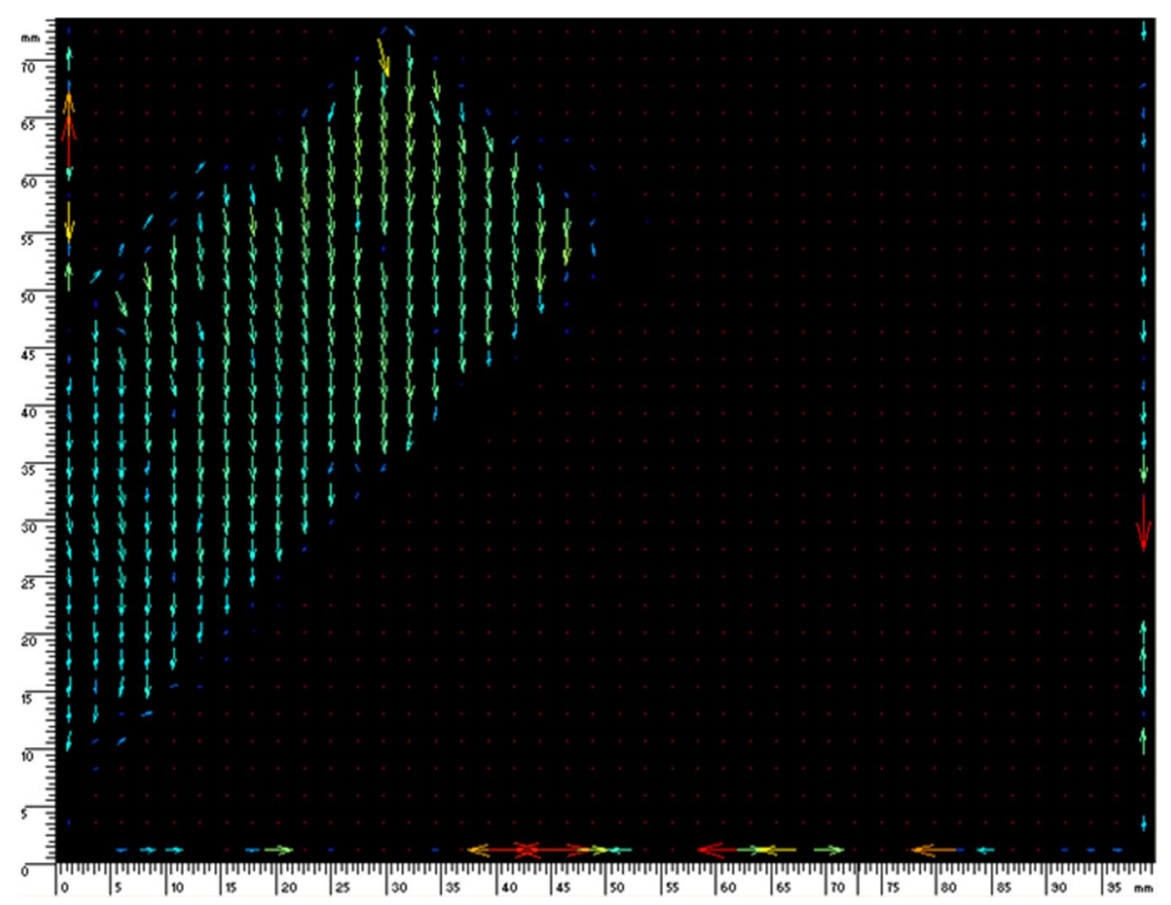

Figure 4.11 PIV velocity profile of flow $2.5 \mathrm{~mm}$ above the channel floor between the 6th and 7 th tumble strips at $2.0 \mathrm{~m} / \mathrm{s}$ exit flow velocity.

Results of the inter-tumble strip measurement is shown in Figure 4.11. For this set of measurements, digital masking of the camera images prior to correlation processing of vectors was also used to eliminate the generation of PIV measurements in regions not illuminated by the light sheet.

\section{Summary}

The series of tests was beneficial in developing an initial understanding of PIV measurement principles. Initially, reasonable measurements could not be achieved due to the saturation of tracer particles produced by the large Dantec seeder; however, this issue was overcome with the fabrication of a small, single nozzle seeding system. The testing also provided critical insight into light control techniques critical to making measurements where the measurement plane, and therefore the light sheet, is close to boundary walls in the flow structure. 


\subsection{Stereo PIV of Non-Reacting and Reacting Flows in a Model Gas Turbine Combustor}

\section{Objective}

The objective of this test was to first successfully set up the PIV system for stereo PIV measurements and capture the strong swirling flow characteristic to a gas turbine combustor under non-reacting operating conditions. The second objective was to capture the swirling flow profile of the combustor under reacting flow conditions using a combination of liquid and solid seeding particles.

\section{Motivation}

In order to achieve high efficiency and ultra-low emissions, a variety of combustion schemes have been applied to modern advanced gas turbine combustors. They are particularly designed to operate in an ultra lean premixed mode to achieve low nitric oxide (NO) emissions (11). Stratagies to achieve the low NOx emissions are largely focused around fuel-air delivery techniques. Lean-premixed/prevaporized (LPP) combustors demonstrate much lower concentrations of emissions while operating under the fuel-lean condition (11-12). This method is particularly important in gas-propelled combustors. The strategy behind utilizing the lean-burn mode is to reduce the flame temperature, which is a primary factor in control of emissions formation. According to the Arrhenius equation temperature is an exponential function in describing the rate of reaction, and thus slighly higher temperatures yield significantly increased species production rates (13).

Swirlers are designed for the improvement of flame stabilization and turbulent mixing to enhance combustion efficiency and reduce emissions. Swirling flow produces highly turbulent and complex flow and is essential for the lean-burn premixed flame operation. Indeed, swirl injectors have been widely implemented for dominant flame stabilization by producing a central recirculation zone (CRZ) immediately after the dump plane (14-15). 
High shear stresses and strong turbulence intensity are common characteristics of the centralw recirculation zone as a product of strong vortex breakdown (13). Combustion affects the shape of the recirculation zone and increases turbulence intensity to induce large changes in the aerodynamics of swirling flow fields (13). Low $\mathrm{NO}_{\mathrm{x}}$ emissions result from the low flame temperature with homogeneity of fuel-air mixture. The effect of swirl on emissions is largely dependent on the specific swirl configuration. Increasing swirl generates a larger CRZ to entrain more air that cools down combustion products, reducing thermal NO production. However the introduction of swirling smaller scale mixing enhances the local chemical reaction, producing higher temperatures that results in higher NO formation rate. Meanwhile, increasing swirl strength also increases local oxygen availability and turbulence intensity to promote NO formation.

Boxx et al. (16) published results of temporally resolved stereo PIV and OH* PLIF measurements on a $10 \mathrm{~kW}$ partially premixed, swirl stabilized, methane-air combustor. The combustor was seeded using $0.5 \mu \mathrm{m}$ nominal diameter $\mathrm{TiO}_{2}$ solid seeding particles. The time resolved measurements reviled a CRZ composed of small transient vortices produced by shear layers between the CRZ and oncoming combustible mixture. The research revealed that the reaction zone resided primarily at this interface, while being stabilized by the intense mixing produced by the transient vortices between the hot combustion products in the IRZ and fresh incoming fuel.

Sadanandan et al. (17) published a paper in 2008 based on PIV and OH-PLIF measurements made on a swirled methane-air atmospheric $10.3 \mathrm{~kW}$ combustor running at a global equivalence ratio of 0.75 . PIV measurements were again made using $\mathrm{TiO}_{2}$ as a seeding medium. In their PIV measurements, the researchers observed both a significant CRZ as well as outer recirculation zone (ORZ) in the reacting flow as well as intense mixing from temporal vortices generated in shear layers. OH-PLIF measurements also revealed high $\mathrm{OH}$ concentration gradients in these regions, effectively used to detect the reaction zone. 
The focus of this work is an initial experimental characterization of the flame behavior for an improve understand the flame ignition process and interaction of flow field to heat release. First, the purpose of this study is to improve our understanding of the swirling flame by measuring the detailed flow structure near the dump plane in a model gas turbine combustor. The three velocity fields have been measured using a 3-D stereo PIV system for both the non-reacting (isothermal) and reacting flow. Simultaneous measurement of the velocity field with flame chemiluminescence is able to provide information on flame ignition processes influenced by the local velocity field. Furthermore, maximum heat release can be correlated with the velocity field to obtain information on the heat release process after the flame ignition.

The second purpose of this study is to compare the flow structures by varying the residence time and overall/global equivalence ratios. Understanding and correlation of the fundamental data obtained from the non-reacting flow will leverage the prediction of flame ignition and propagation mechanisms occurring in reacting flow and their differences will be addressed in this study. The velocity data obtained will serve as benchmark data for the validation and improvement of CFD modelling to characterize the complex swirling flow. The development of advanced low emissions combustor concepts to meet increasingly stringent NOx emission goals is expected to increase the probability of encountering combustion dynamics issues, such as the flame ignition, stabilization, and combustion instability.

\section{Hardware Setup}

A diagram of the model gas turbine combustor is shown in Figure 4.12. The combustor consists of three sections: a mixing chamber, swirler-injector body, and sudden-expansion combustor. The swirler is composed of ten straight, flat vanes oriented at 45 degrees with respect to the oncoming air, inducing a swirl number of approximately 0.69 to the air flow (5). The swirling air passes the annulus and mixes with the fuel spray. Methane $(99.9 \%)$ as a fuel is horizontally injected through ten $1.27-\mathrm{mm}(0.05$-in.) diameter holes as shown in Figure 4.12. The fuel injector holes are located just downstream of the 
swirler ensuring rapid mixing. Methane enters through the centerline of the swirlerinjector body and the flow rate is precisely controlled by a mass flow meter [MKS model].

Compressed air, also controlled through a mass flow meter, enters at the mixing chamber surrounding the swirler-injector body creating a cross-jet fuel injection flow configuration. The mixing chamber, packed with glass beads (diameter $\sim 3 \mathrm{~mm}$ ), relaxes the on-coming air producing a relatively uniform flow velocity. Since the combustor was designed to provide different levels of mixing between fuel and air by varying the location of fuel injection, the mixing chamber is mainly designed to achieve full mixing prior to the flow reaching the sudden-expansion combustor. The fuel injection locations for the short and long residence cases are $11.4 \mathrm{~mm}$ (0.45 in.) and $21.6 \mathrm{~mm}$ (0.85 in.) from the dump plane respectively.

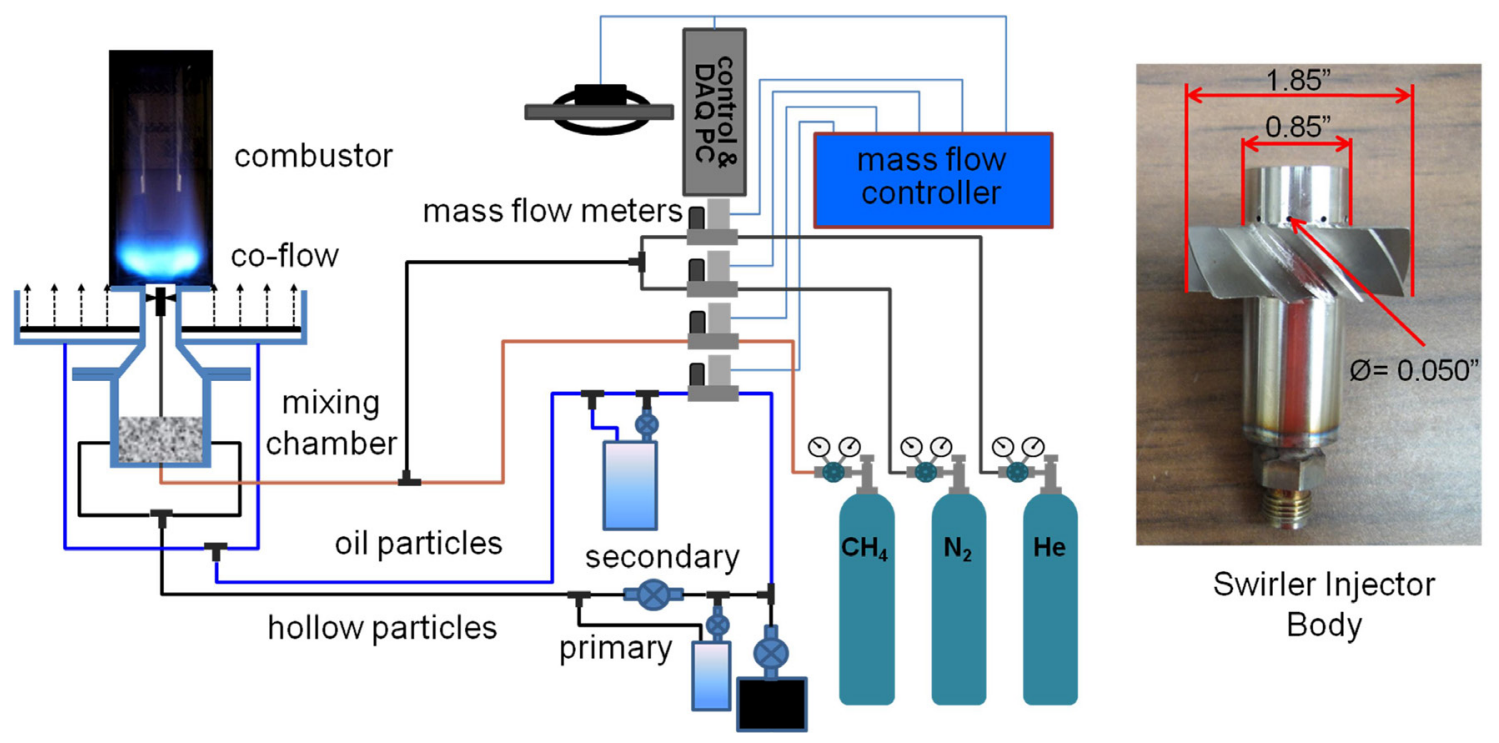

Figure 4.12 Model gas turbine combustor schematic and swirler injector body. 


\section{PIV Setup}

The stereo PIV system is shown in Figure 4.13. PIV components consists of a dual cavity Nd:YAG laser, beam guide with sheet optics, dual camera system, and control unit. Two different seeding particle generators were employed for the PIV measurements, one for oil particles and one for spherical hollow glass particles. Oil particles (DEHS) are seeded through the co-flow section with minimal flow rate while the hollow particles are fed through the combustor annulus gap. The nominal sizes are 1 $\mu \mathrm{m}$ and $11 \mu \mathrm{m}$ for the oil and glass particles. Most of the glass particles survive through the flame while oil particles quickly vaporize when approaching the flame zone. A small fraction of the hollow glass particles burn, producing a yellowish flame. However, the structure of the flame remains unchanged.

A forward scatter PIV camera configuration was chosen for multiple reasons. The primary benefit of this configuration was a strong particle signal based on Mie light scattering principles. A strong particle image signal is required in the case of reacting flow due to the flame's luminosity which can wash out a weak PIV particle signal. The second benefit of this configuration was the ability to place the ICCD (DicamPro) camera orthogonal to the light sheet with an unobstructed view to capture flame luminescence. For the flame chemiluminescence, an ICCD camera was employed with an interference filter of $431 \mathrm{~nm}$ to detect $\mathrm{CH}^{*}$ chemiluminescence. A consumer digital camera was used for flame monitoring purpose as well.

Dantec Dynamics' FlowManager software provided 3 component velocity maps and Matlab was used for further visualization and analysis of both PIV vector profiles and flame chemiluminescence imaging. The DicamPro camera used for chemiluminescence was triggered using the same trigger pulse sent to the PIV cameras. Camera control was provided using PCO CamWare software. The camera was configured to open the gate immediately following the second laser pulse with a gate width time of $1 \mathrm{~ms}$. The $60 \mathrm{~mm}$ camera focal lens was used at an F-stop of 2.4 for an approximate focal depth of $2 \mathrm{~mm}$. 


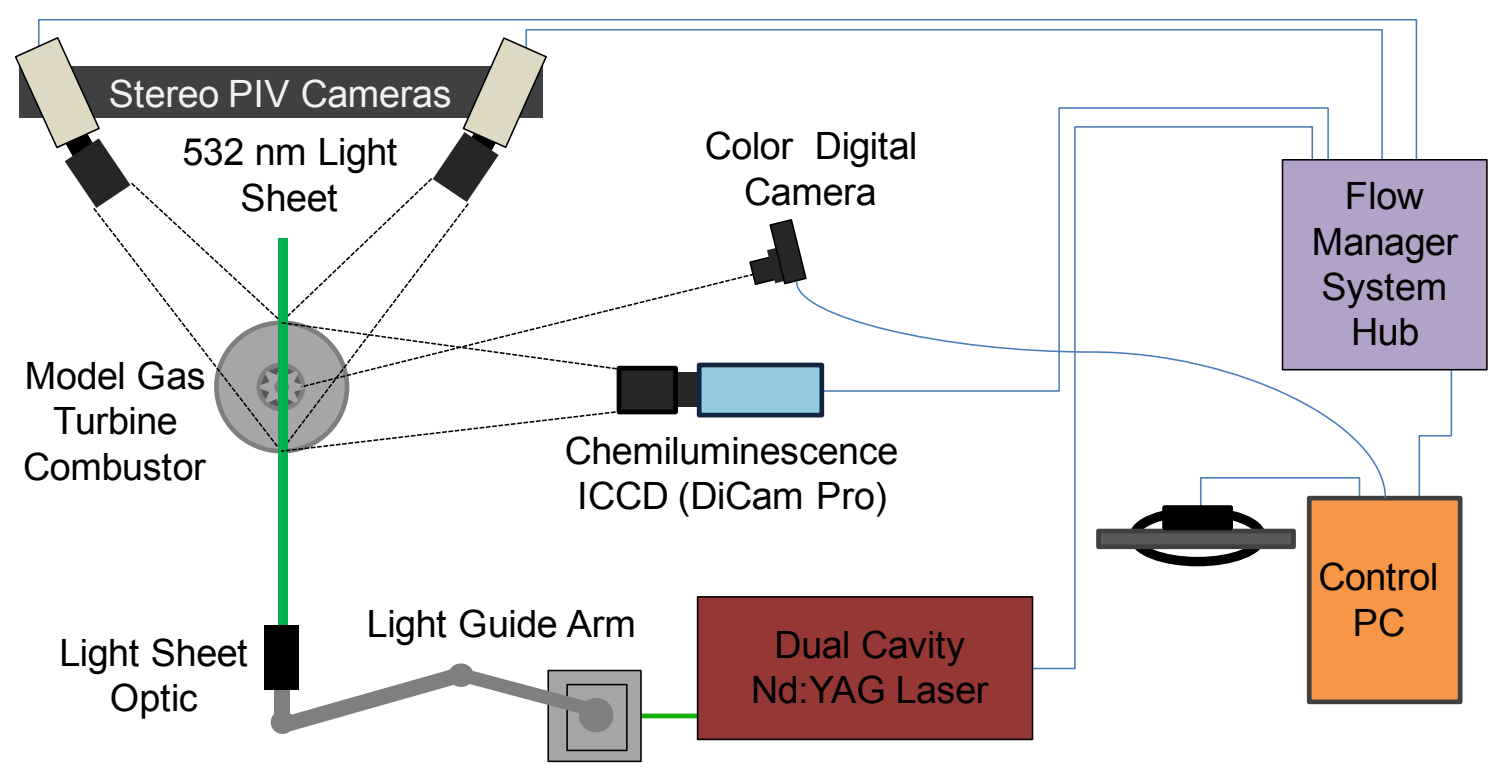

Figure 4.13 Schematic of stereo PIV setup.

The seeder was designed based on the combination of several solid particle generation techniques for the seeding of PIV flows. The seeder implements key features of both fluidized bed type seeders and cyclone based seeders. In addition, two additional techniques were integrated to aid in consistent and uniform particle generation; mechanical vibration and anti-clumping beads. A schematic of the hybrid solid particle seeder is shown in Figure 4.14.

As the schematic shows, the air supply to the seeder is split in two, with part of the flow diverted to the bottom of the seeder, below a porous filter, creating a fluidized bed as it passes up through the filter and hollow glass sphere (Sigma-Aldrich PN 440345) particle bed. Agitation of the seeding particles, provided by the rigid attachment of a shakermotor system to the body of the seeder, collapses any paths created by the seeding air as it passes through the particle bed. This ensures that new paths are constantly being created resulting in seeding particles being picked up by the air flow during the creation of new paths. Three millimeter diameter beads are also mixed with the hollow glass sphere seeding particles to prevent clumping of the seeding medium. The rest of the air 
flow to the seeder is routed to the top of the seeder, producing a cyclone as the flow travels downward and around the seeder body to the particle bed. Forcing the air flow to travel down to the particle bed helps the flow pick up particles on the surface of the particle bed, while the cyclone nature of the flow prevents large particle clumps from entering the central exit tube.

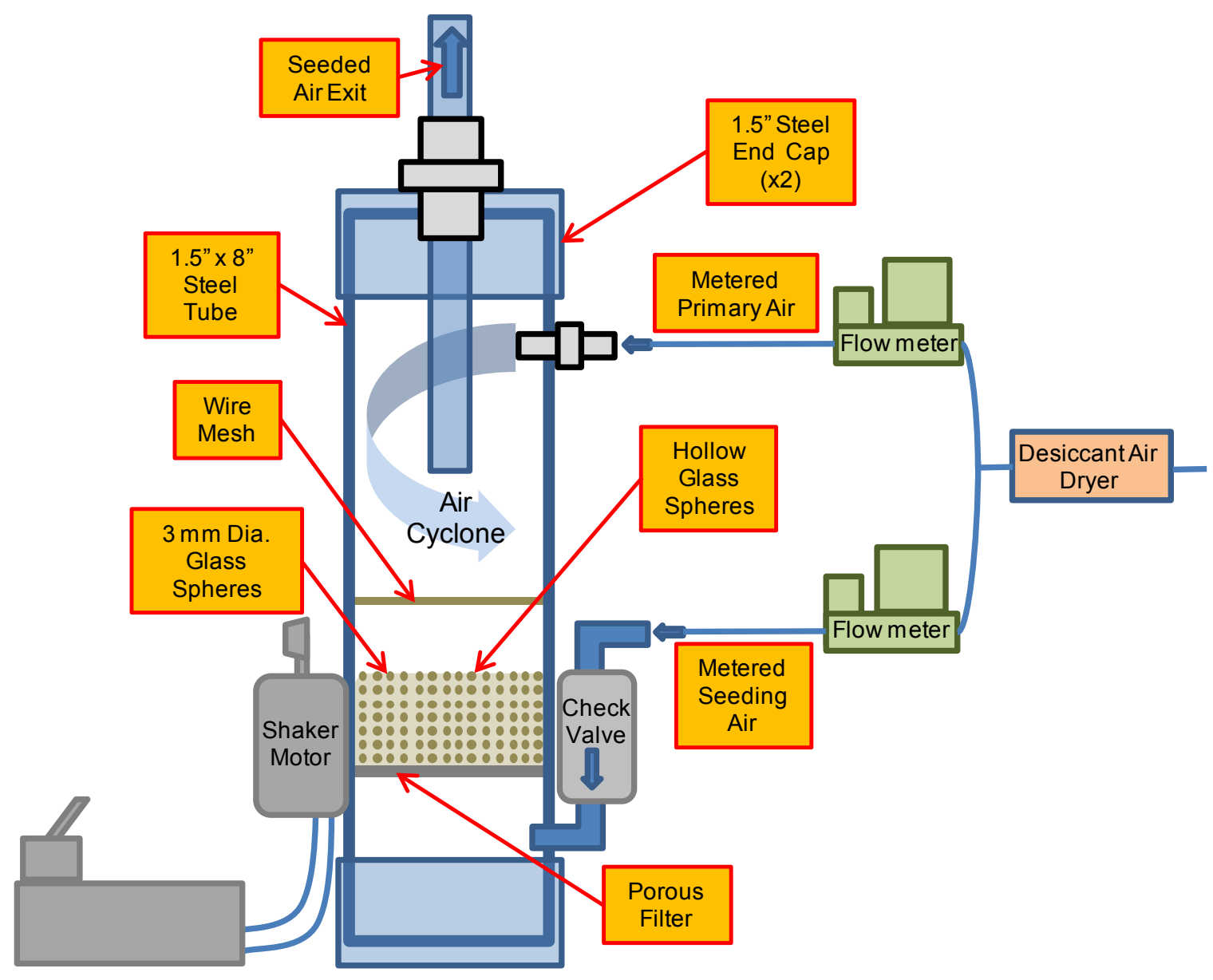

Schematic not to scale

Figure 4.14 Hybrid fluidized bed - cyclone type solid particle seeder for seeding of hollow glass sphere tracer particles. 
Processing of the raw PIV images into velocity vector maps was performed using Dantec Dynamics FlowManager software environment as well as MatLab. Derivation of velocity vectors from the raw images began with the application of an adaptive correlation processing algorithm. The adaptive correlation consists of a cross-correlation algorithm applied using multiple iterations and the ability to offset the second interrogation region. The selected interrogation region was a two-step process, starting as a 128 x 128 pixel window with a final window size of 32 x 32 pixels.

For each step three iterations were used for placement of the offset second interrogation region. A 50\% overlap in interrogation regions was also applied for higher vector resolution. Due to the small diameter of the DEHS oil seeding particles, a low-pass Gaussian filter was applied to the raw images with a k-value of three. Peak validation was also applied using a peak width range of $2-6$ pixels and a minimum peak height relative to peak 2 of 1.2. Central differencing was used for interrogation area offset as well as the application of high accuracy subpixel refinement calculations.

Once the velocity vectors were calculated using the preceding adaptive correlation processing setup, a range validation check was applied, allowing for valid vector lengths of $0-6 \mathrm{~m} / \mathrm{s}$. The range validation was followed by triple iteration $3 \times 3$ vector moving average validation with an acceptance factor of 0.1 . The moving average validation was then followed by a final $3 \times 3$ vector averaging filter. FlowManager 3-D PIV software was then used, where a calibration process was applied to align the separate 2-D images, correct for perspective distortions, and calculate a finalized 3 component vector field. The last step in the PIV imaging was to export the 3 component velocity maps into the MatLab environment for visualization, interpretation, and quantitative analysis. 


\section{Test Conditions}

Table 4.1 shows the parameters of flow investigated for both non-reacting and reacting flows composed of four conditions for non-reacting flow and eight for reacting flame. The control parameters include different residence times of fuel and air by displacing the location of fuel injection and global equivalence ratio. Two global equivalence ratios of 0.5 and 0.6 are considered with a thermal power of 4.7 and $5.7 \mathrm{~kW}$.

Table 4.1

Test conditions for gas combustor.

\begin{tabular}{|c|c|c|c|c|c|c|c|c|}
\hline $\begin{array}{c}\text { Test } \\
\text { Number }\end{array}$ & Swiler Body & $\begin{array}{l}\text { Primary } \\
\text { Seed }\end{array}$ & Flow Type & $\begin{array}{c}\text { Air } \\
\text { Flow }\end{array}$ & Fuel & $\begin{array}{l}\text { Fuel } \\
\text { Flow }\end{array}$ & Phi & $\begin{array}{l}\text { Image } \\
\text { Count }\end{array}$ \\
\hline 1 & $\begin{array}{c}\text { Short Residence } \\
\text { Swirler } \\
\end{array}$ & No & Rxn Flow & $\begin{array}{l}150 \\
\text { SLM }\end{array}$ & $\mathrm{CH}_{4}$ & $\begin{array}{l}7.95 \\
\text { SLM } \\
\end{array}$ & 0.5 & $\begin{array}{c}300 \\
\text { Images }\end{array}$ \\
\hline 2 & $\begin{array}{l}\text { Short Residence } \\
\text { Swirler }\end{array}$ & Yes & Rxn Flow & $\begin{array}{l}150 \\
\text { SLM }\end{array}$ & $\mathrm{CH}_{4}$ & $\begin{array}{l}7.95 \\
\text { SLM }\end{array}$ & 0.5 & $\begin{array}{c}300 \\
\text { Images }\end{array}$ \\
\hline 3 & $\begin{array}{l}\text { Short Residence } \\
\text { Swirler }\end{array}$ & No & Rxn Flow & $\begin{array}{l}150 \\
\text { SLM }\end{array}$ & $\mathrm{CH}_{4}$ & $\begin{array}{l}9.54 \\
\text { SLM }\end{array}$ & 0.6 & $\begin{array}{c}300 \\
\text { Images }\end{array}$ \\
\hline 4 & $\begin{array}{c}\text { Short Residence } \\
\text { Swirler }\end{array}$ & Yes & Rxn Flow & $\begin{array}{l}150 \\
\text { SLM }\end{array}$ & $\mathrm{CH}_{4}$ & & 0.6 & $\begin{array}{c}300 \\
\text { Images }\end{array}$ \\
\hline 5 & $\begin{array}{c}\text { Short Residence } \\
\text { Swirler }\end{array}$ & Yes & $\begin{array}{l}\text { Non Rxn } \\
\text { Flow }\end{array}$ & $\begin{array}{l}150 \\
\text { SLM }\end{array}$ & $\mathrm{N}_{2} / \mathrm{He}$ & $\begin{array}{l}7.95 \\
\text { SLM }\end{array}$ & 0.5 & $\begin{array}{c}300 \\
\text { Images }\end{array}$ \\
\hline 6 & $\begin{array}{l}\text { Short Residence } \\
\text { Swirler }\end{array}$ & Yes & $\begin{array}{l}\text { Non Rxn } \\
\text { Flow }\end{array}$ & $\begin{array}{l}150 \\
\text { SLM }\end{array}$ & $\mathrm{N}_{2} / \mathrm{He}$ & $\begin{array}{l}9.54 \\
\text { SLM }\end{array}$ & 0.6 & $\begin{array}{c}300 \\
\text { Images }\end{array}$ \\
\hline 7 & $\begin{array}{l}\text { Long Residence } \\
\text { Swirler }\end{array}$ & No & Rxn Flow & $\begin{array}{l}150 \\
\text { SLM }\end{array}$ & $\mathrm{CH}_{4}$ & $\begin{array}{l}7.95 \\
\text { SLM }\end{array}$ & 0.5 & $\begin{array}{c}300 \\
\text { Images }\end{array}$ \\
\hline 8 & $\begin{array}{l}\text { Long Residence } \\
\text { Swirler }\end{array}$ & Yes & Rxn Flow & $\begin{array}{l}150 \\
\text { SLM }\end{array}$ & $\mathrm{CH}_{4}$ & $\begin{array}{l}7.95 \\
\text { SLM }\end{array}$ & 0.6 & $\begin{array}{c}300 \\
\text { Images }\end{array}$ \\
\hline 9 & $\begin{array}{l}\text { Long Residence } \\
\text { Swirler }\end{array}$ & No & Rxn Flow & $\begin{array}{l}150 \\
\text { SLM }\end{array}$ & $\mathrm{CH}_{4}$ & $\begin{array}{l}9.54 \\
\text { SLM }\end{array}$ & 0.6 & $\begin{array}{c}300 \\
\text { Images }\end{array}$ \\
\hline 10 & $\begin{array}{c}\text { Long Residence } \\
\text { Swirler }\end{array}$ & Yes & Rxn Flow & $\begin{array}{l}150 \\
\text { SLM }\end{array}$ & $\mathrm{Ch}_{4}$ & $\begin{array}{l}9.54 \\
\text { SLM }\end{array}$ & 0.6 & $\begin{array}{c}300 \\
\text { Images }\end{array}$ \\
\hline 11 & $\begin{array}{l}\text { Long Residence } \\
\text { Swirler }\end{array}$ & Yes & $\begin{array}{l}\text { Non } \operatorname{Rxn} \\
\text { Flow }\end{array}$ & $\begin{array}{l}150 \\
\text { SLM }\end{array}$ & $\mathrm{N}_{2} / \mathrm{He}$ & $\begin{array}{l}7.95 \\
\text { SLM }\end{array}$ & 0.5 & $\begin{array}{c}300 \\
\text { Images }\end{array}$ \\
\hline 12 & $\begin{array}{l}\text { Long Residence } \\
\text { Swirler }\end{array}$ & Yes & $\begin{array}{l}\text { Non Rxn } \\
\text { Flow }\end{array}$ & $\begin{array}{l}150 \\
\text { SLM }\end{array}$ & $\mathrm{N}_{2} / \mathrm{He}$ & $\begin{array}{l}9.54 \\
\text { SLM }\end{array}$ & 0.6 & $\begin{array}{c}300 \\
\text { Images }\end{array}$ \\
\hline
\end{tabular}




\section{Results and Discussion}

The 3-component stereo PIV velocity map of the non-reacting flow corresponding to Test 12 and the reacting flow corresponding to Test 10 can be seen in Figure 4.15, where the arrows denote the $u$ and $v$ components of the velocity and the color map denotes the $w$ component with red denoting flow out of the image plane and blue flow into the plane. The time averaged PIV measurement shows a highly symmetrical swirled flow with a large CRZ seen between the strong out of plane velocity components.

In the time averaged reacting flow case, there are several notable differences in the flow structure compared to the non-reacting flow. As expected, there is an increase in the flow velocity due to the heat release of combustion and thermal expansion of gases. The $u$ component of the flow, i.e., radial flow velocity, is significantly increased as well as the $v$ component. For the reacting flow, the CRZ width has diminished significantly and experiences greater velocity gradients. The high velocity gradients result in shear layers where previous work by others has shown small temporal vortices to be created and destroyed. These vortices are responsible for the mixing of heated combustion products and fresh reactants.

In the instantaneous non-reacting flow velocity profile of Test 11 shown in Figure 4.16, smaller staggered vortices can be seen that make up the CRZ along with higher velocity gradients than the time averaged case as shown in Figure 4.15 responsible for creating the transient vortices. Figure 4.16 (bottom) shows a single 3-component velocity profile of the reacting flow corresponding to Test 8 . Here, a very sharp velocity gradient can be seen between the CRZ and out coming flow. 

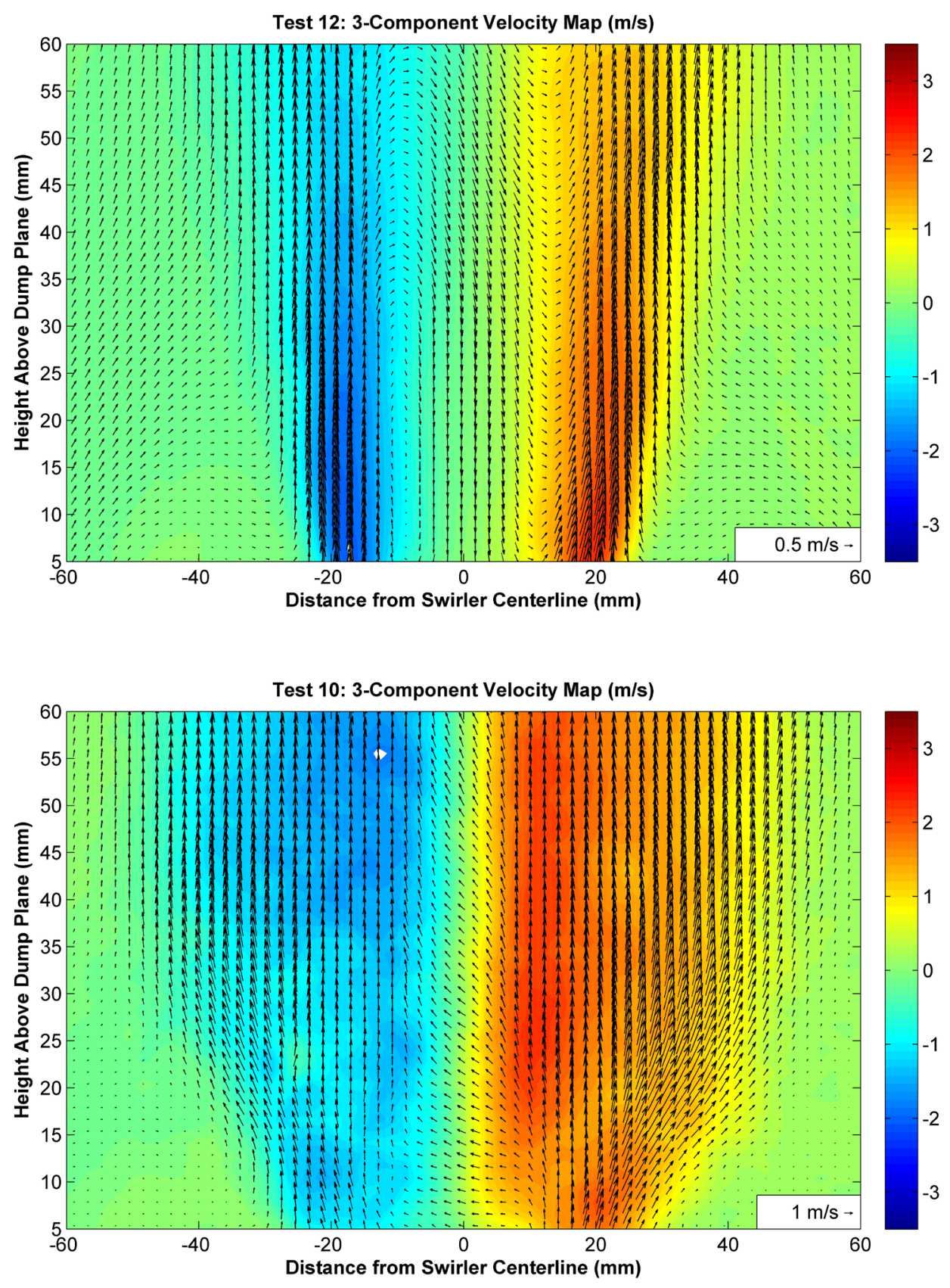

Figure 4.15 Time averaged velocity fields for the non-reacting flow of Test 12 (top) and the reacting flow of Test 10 (bottom). 

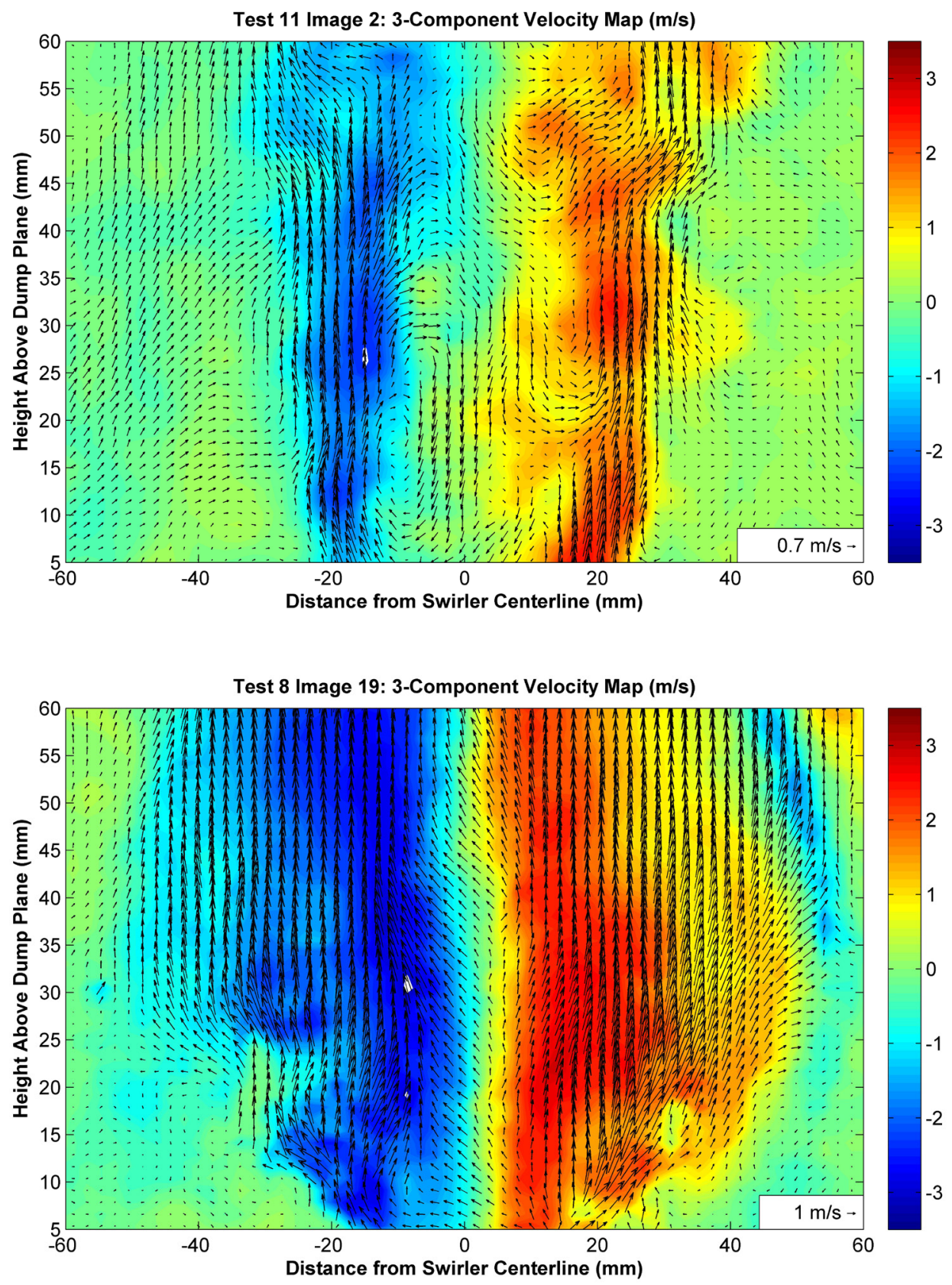

Figure 4.16 Instantaneous velocity fields for the non-reacting flow of Test 11 (top) and the reacting flow of Test 8 (bottom). 
Comparison of the two instantaneous measurement cases shows a distinct decrease in the CRZ for the case of reacting flow as well as greater velocity gradients at the CRZ boundary. The outer profile of the swirling flow however, shows lower velocity gradients compared to the non-reacting flow case, possibly due to convective currents provided by the thermal heat release of the reacting flow.

The averaged $u$ and $v$ components of the velocity field have been processed and plotted for further interpretation and comparison of flow across test cases. Due to the large degree of radial symmetry in the $u$ and $v$ components of the flow fields, each vector map has been divided in half along the combustor centerline, allowing the flow under different test conditions to be compared side by side. The central recirculation zone for each vector map has been colored for ease of visualization, with different colors used to separate the two test cases being compared.

Figure 4.17 shows the averaged $u$ and $v$ velocity components for Test 5 (top left), Test 11 (top right), Test 6 (bottom left), and Test 12 (bottom right) for the non-reacting flow conditions. As shown in Figure 4.17, the long residence swirler produces slightly higher axial velocities at the dump plane, while the axial velocities away from the dump plane are comparable to the short residence cases. The CRZ for the non-reacting flow case exhibits slightly greater axial velocities near the dump plane in the case of the short residence swirler for both equivalence ratios. However, in both cases, the long residence swirler produces a broader CRZ compared to the short residence swirler as shown in color. The radial and axial size of CRZ is smaller for short residence case when examining the velocity vector field closely near the combustor centerline.

For the reacting flow shown in Figure 4.18, the long residence swirler produces both a broader and higher axial velocity flow field compared to the short residence swirler for both equivalence ratios. An increase in both the axial and radial velocities for the long residence swirler indicates greater heat release and thermal expansion of the combustion product gases near the dump plane of the combustor compared to the short residence configuration. 

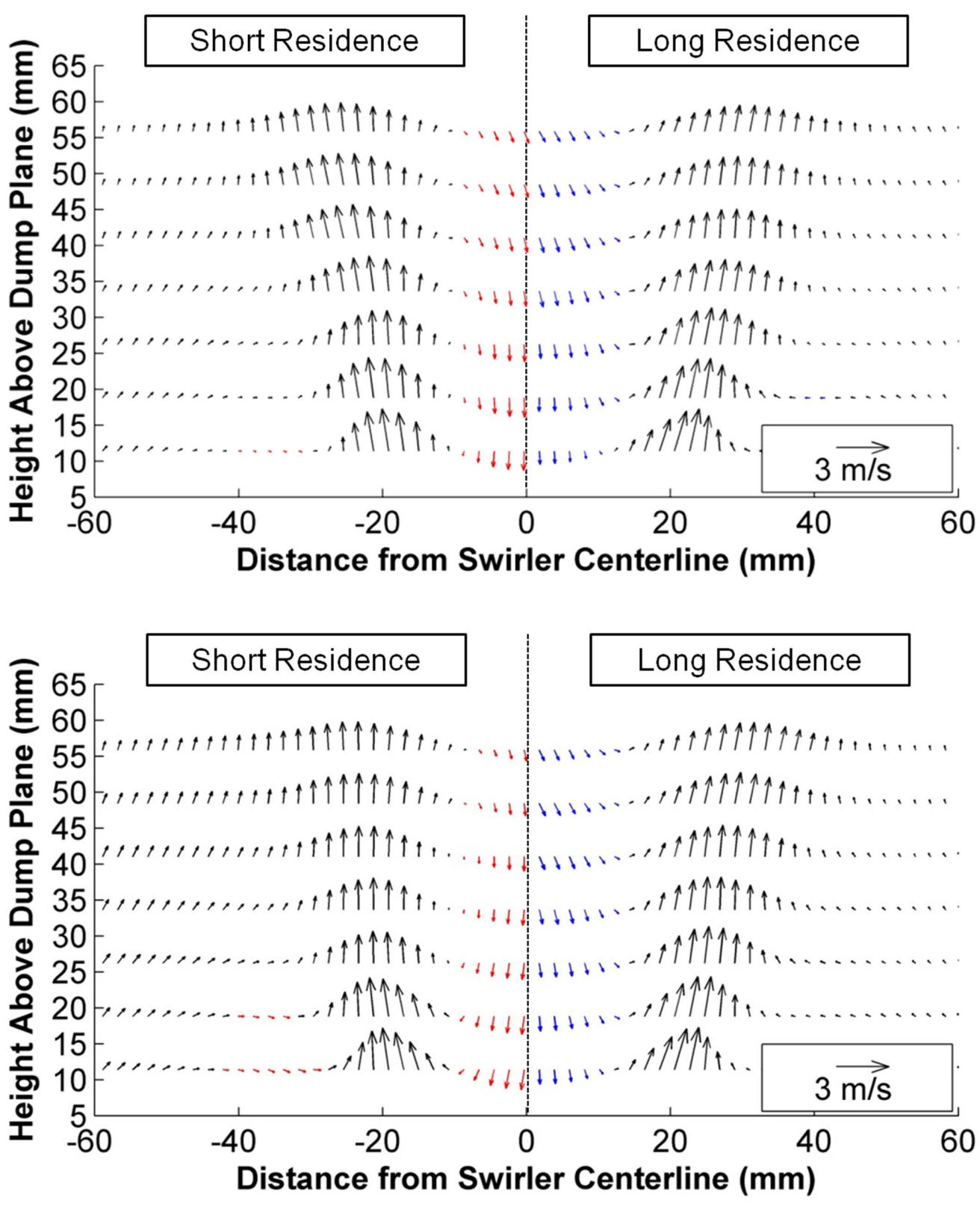

Figure 4.17 Averaged $\mathrm{u}$ and $\mathrm{v}$ velocity components for Test 5 (top left), Test 11 (top right), Test 6 (bottom left), and Test 12 (bottom right) for the non-reacting flow conditions. 

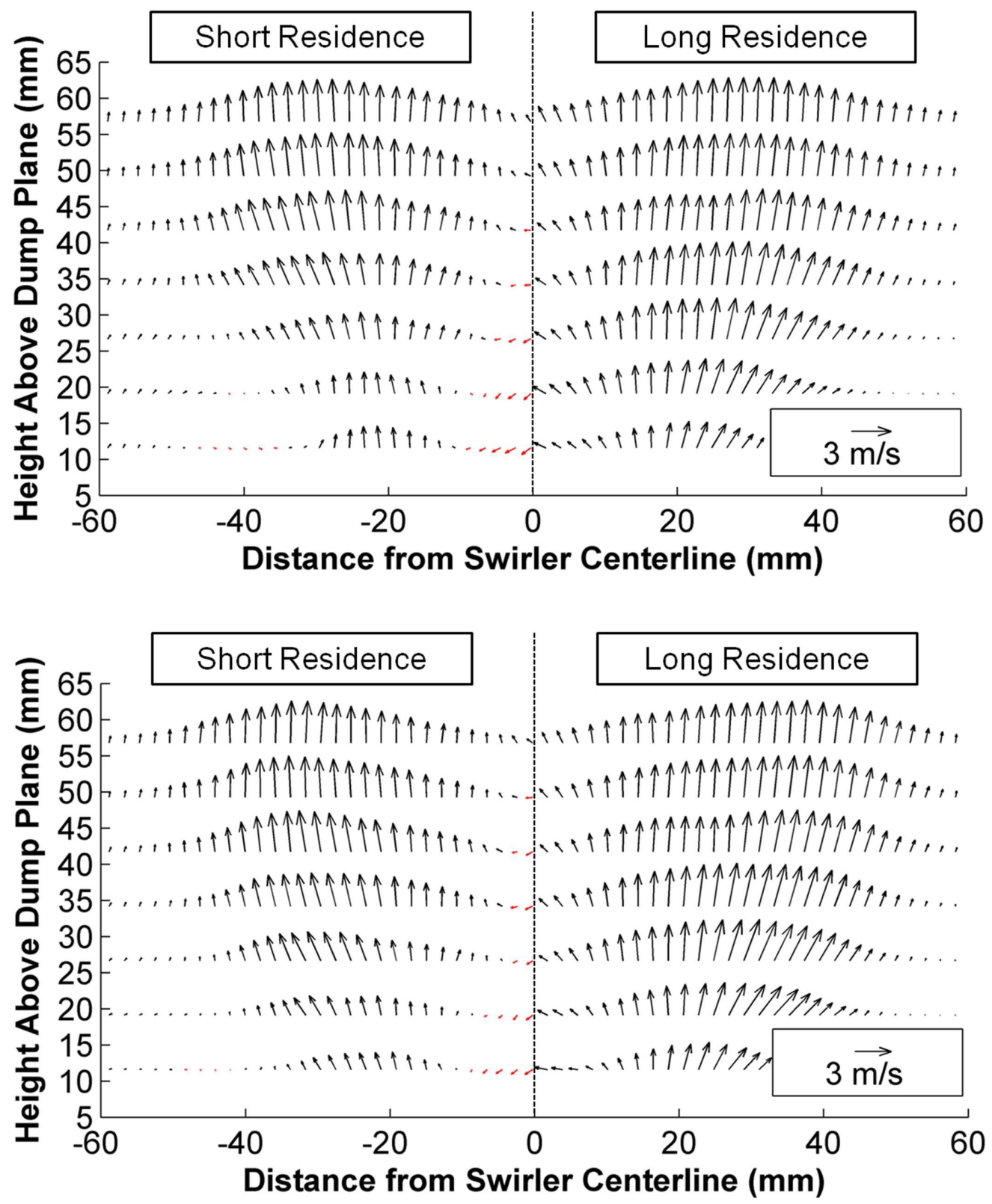

Figure 4.18 Averaged $\mathrm{u}$ and $\mathrm{v}$ velocity components for Test 2 (top left), Test 8 (top right), Test 4 (bottom left), and Test 10 (bottom right) for the reacting flow conditions. 
For both the short and long residence swirler applications, the exterior edges of the velocity profiles exhibit a more gradual tapper to the co-flow velocity. This taper is likely due to the increase in heat release, producing greater thermal heat release and convective flow around the flame. Comparison of the reacting flow profiles to their counterpart non-reacting flow profiles shows a stark difference in the CRZ. The degradation in CRZ could be attributed to greater fluctuation in the flow profile.

Figure 4.19 shows the instantaneous chemiluminescence images for Test 2, Test 4, Test 8 , and Test 10 . First of all, the flames captured in the study show highly convoluted and wrinkled flame, which is representative of the typical turbulent flame. The flame position in Test 2 exhibited a detached nature with no steady anchor to the combustor's dump plane. On the top right of Figure 4.19, a flame produced by the short residence swirler under a slightly greater fuel flow rate of $9.54 \mathrm{slm} \mathrm{CH}_{4}$ is exhibited. The increase in global equivalence ratio from 0.5 to 0.6 , resulted in flame stabilization and anchoring at the outer edge of the combustor's dump plane annulus. In the bottom left of Figure 4.19, a detached flame is again seen, this time in the case of the long residence swirler with a global equivalence ratio of 0.5 . Although the flame is still detached from the dump plane, it is obvious when comparing the two images on the left hand side that the long residence swirler reduces the level of flame detachment seen by the smaller gap between flame and dump plane. In the bottom right, an example of a stable fully anchored flame is seen as a product of both the longer residence swirler and higher equivalence ratio of 0.6 , indicating the stabilized flame at the dump plane.

The RMS average intensity was calculated for each reacting flow test case, shown in Figure 4.20 below. The RMS image was constructed with the ratio of the fluctuated image to the corresponding averaged images. The level of intensity in the RMS images indicates the level of fluctuation in image intensity, directly corresponding to flame fluctuation, across the 150 images from each test. The dark red zones in the two left hand images corresponding to Test 2 and Test 4 indicate a high level of fluctuation in flame position due to the flame being detached and susceptible to instabilities in the flow. 

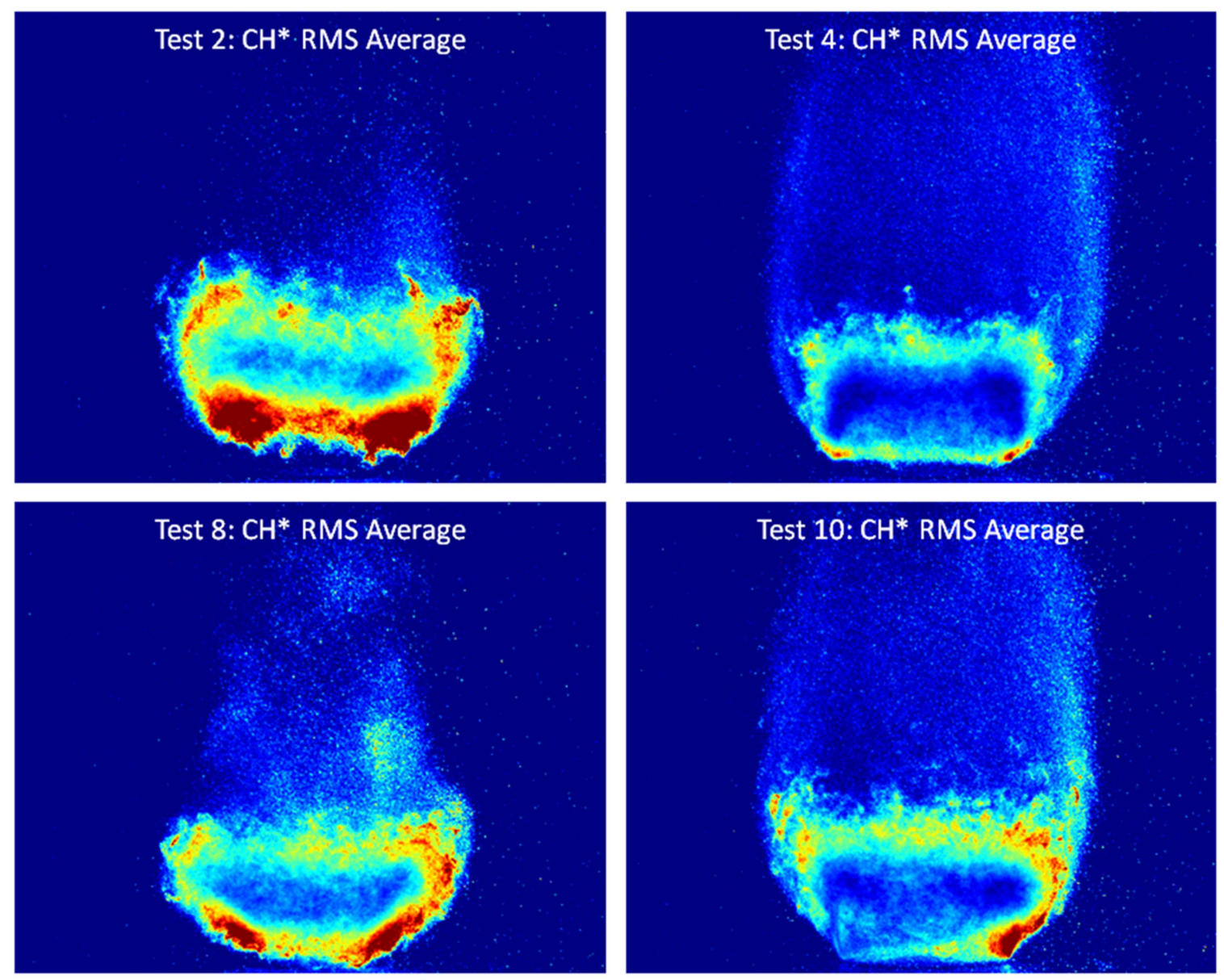

Figure 4.19 False-colored chemiluminescence images for Test 2 (top left), Test 4 (top right), Test 8 (bottom left), and Test 10 (bottom right) for the reacting flow conditions. Red indicates the high intensity while blue indicates the low intensity.

In contrast the images on the right corresponding to the higher fuel flow rate and global equivalence ratio of 0.6 exhibit a lower RMS intensity, indicating lower levels of flame fluctuation, an indication of greater flame stability due to flame anchoring at the dump plane combustor annulus. Comparison of the image based on residence time, short residence vs. long residence comparing the images vertically, also indicates an improvement in flame stability with respect to residence time. 

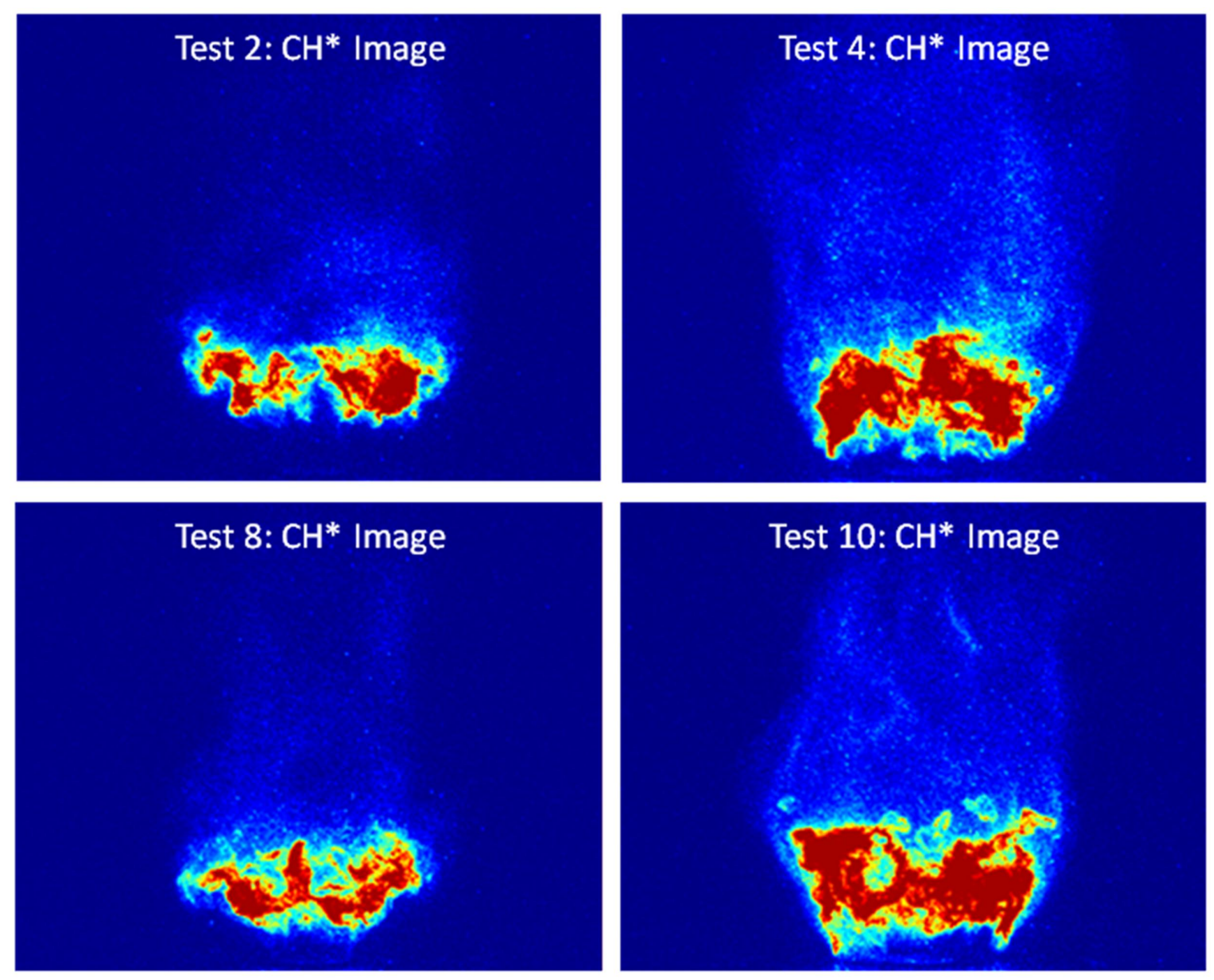

Figure 4.20 False-colored rms chemiluminescence images for Test 2 (top left), Test 4 (top right), Test 8 (bottom left), and Test 10 (bottom right) for the reacting flow conditions. Red indicates the high intensity while blue indicates the low intensity.

Figure 4.21 shows the points for the very first flame ignition locations from the dump plane. The initial flame ignition locations from the instantaneous chemiluminescence images were determined by tracking the chemiluminescence intensity over the radial and axial directions from the dump plane. This identification of flame ignition locations yields general perceptions for the flame to be detached, attached, or symmetric depending upon residence time and equivalence ratio. The calculation was performed over the left and right side at the combustor centerline such that the red symbols indicate the left side of flame ignition locations while the blue symbols show the right side of the flame 
ignition. In addition, the degree of scatter also demonstrates flame fluctuations over the axial and radial directions.

The flame in Test 2 , a combination of partially premixed and diffusion modes, shows instability under the lower equivalence ratio condition. For the effect of equivalence ratio on the flame ignition process in the short residence swirler cases (Test 2 vs Test 4), flames initiate with a high degree of fluctuation, most being detached or lift-off in the equivalence ratio of 0.5 compared to those at the equivalence ratio of 0.6 . In the Test 4 condition, the flames are slightly lift-off over the dump plane but tend to anchor along the annulus gap near the dump plane. In contrast, most flames are initiated near the dump plane regardless of the equivalence ratio for the long residence swirler cases of Test 8 and Test 10. For the Test 8 , most flames tend to be stable just downstream of the bluff-body while for the Test 10, the flames anchor at the annulus rip of the dump plane. For Test 8 and Test 10 , relatively stabilized flame takes place near the dump plane.

In the current flame conditions, a high equivalence ratio provides a condition for stable flame anchoring. In terms of swirler residence time, the long residence swirler provides better flame anchoring. This is consistent with the flow field structure as seen in the PIV vector fields, showing a smaller central recirculation zone in the long residence swirler. The mixing of fresh reactants and combustion products presented in the CRZ promotes combustion rate, causing relatively radial spread burning. Homogeneity of mixing process near the dump plane yields a condition for better burn rate.

Further statistical analysis of the flame ignition locations was performed by leveraging the histogram method. Figure 4.22 represents the axial locations of the flame ignition. For the Test 2 case, the mean axial location of flame is found to be about $12.15 \mathrm{~mm}$ with a standard deviation of $4.32 \mathrm{~mm}$ showing the detached flame and highly fluctuated flames among the four flames considered in the study. For the Test 4 case (a mean height of $2.72 \mathrm{~mm}$ with a standard deviation of $1.95 \mathrm{~mm}$ ), the flames show very stable in terms of axial flame stabilization with a Gaussian distribution. 

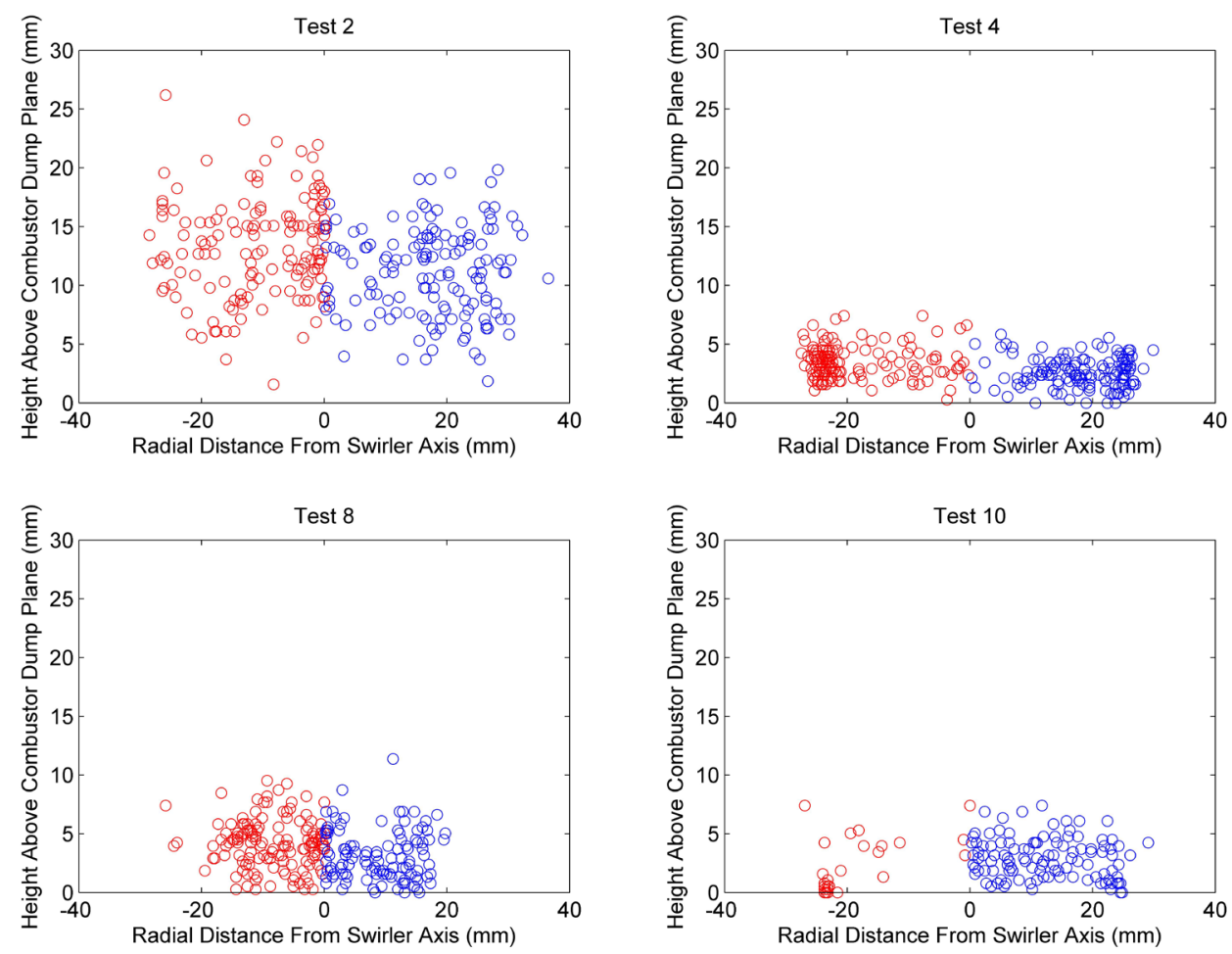

Figure 4.21 Scattering data points for the very initial flame ignition locations for different residence swirlers and equivalence ratios. Test 2: short residence swirler with equivalence ratio of 0.5 , Test 4 : short residence swirler with equivalence ratio of 0.6 .

For Test 8 (a mean height of $2.84 \mathrm{~mm}$ with a standard deviation of $2.88 \mathrm{~mm}$ ), the histogram shows relatively flat while Test 10 (a mean height of $0.66 \mathrm{~mm}$ with a standard deviation of $2.57 \mathrm{~mm}$ ) exhibits flame anchoring at the dump plane without significant flame fluctuations. Test 10 shows stable flames, predominantly attached at the dump plane. Furthermore, the effect of equivalence ratio is more pronounced in terms of flame stabilization compared to residence time. This implies the current flame is more subject to the premixed flame mode and the degree of mixing between fuel and air is critical to stabilizing the flame under the lean-burn mode. Further measurement of the degree of mixedness is necessary to characterize the flame stabilization mechanisms. 
An analysis of the radial locations of flame ignition was performed similar to the axial positions in Figure 4.22. Figure 4.23 shows flames from Tests 4 and 10 tend to anchor near the annulus rip while the flames from Test 8 anchor near the bluff-body. Flames from Test 2 show radial spread burning with detached flames. In the low equivalence ratio cases, the IRZ plays an important role in stabilizing the flame as shown in Test 8 in Figure 4.23. For the detached flame case of Test 2, the swirling motion distributes the initial flame ignition point radially.
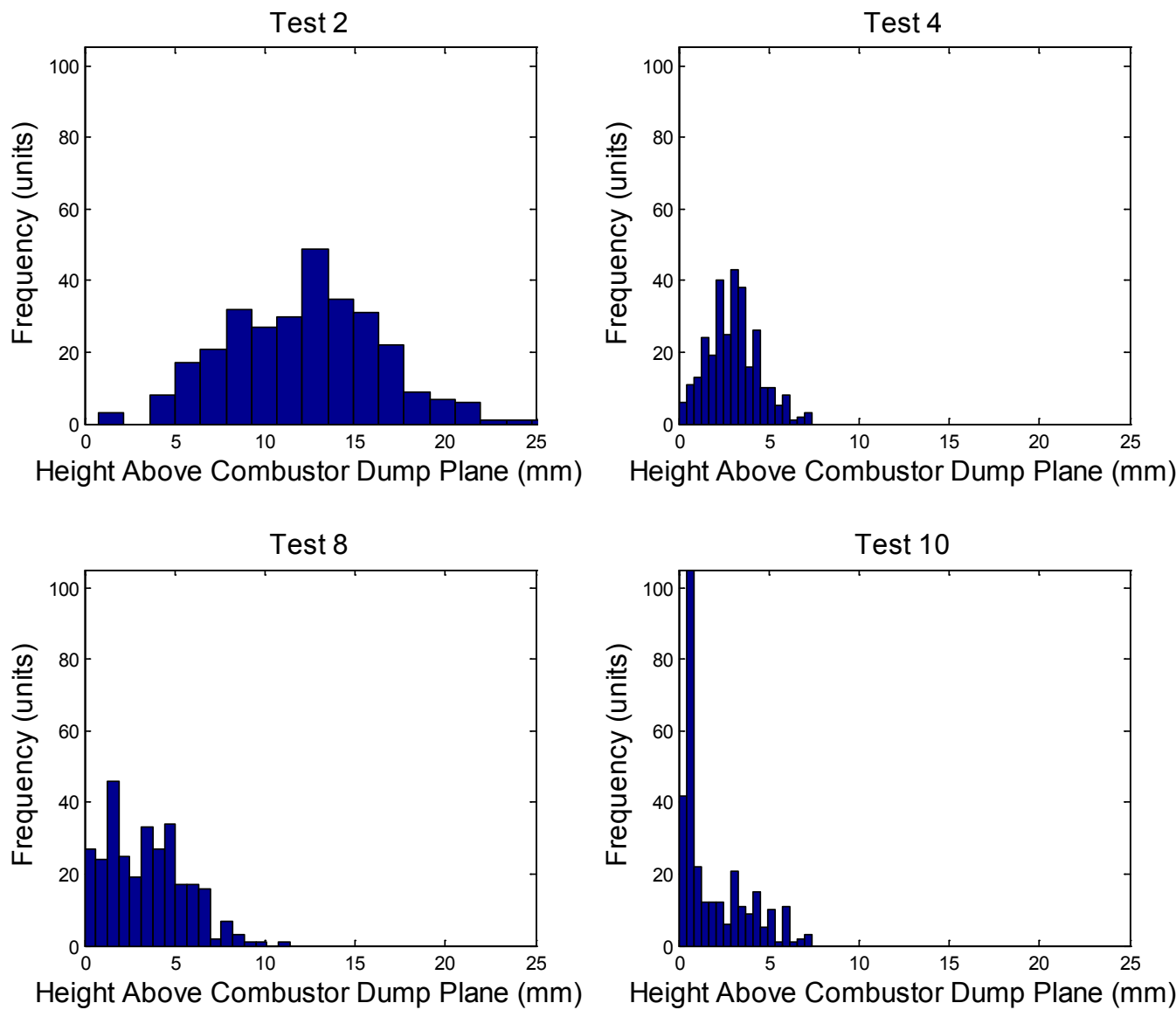

Figure 4.22 Histogram (frequency) of axial flame ignition locations. 

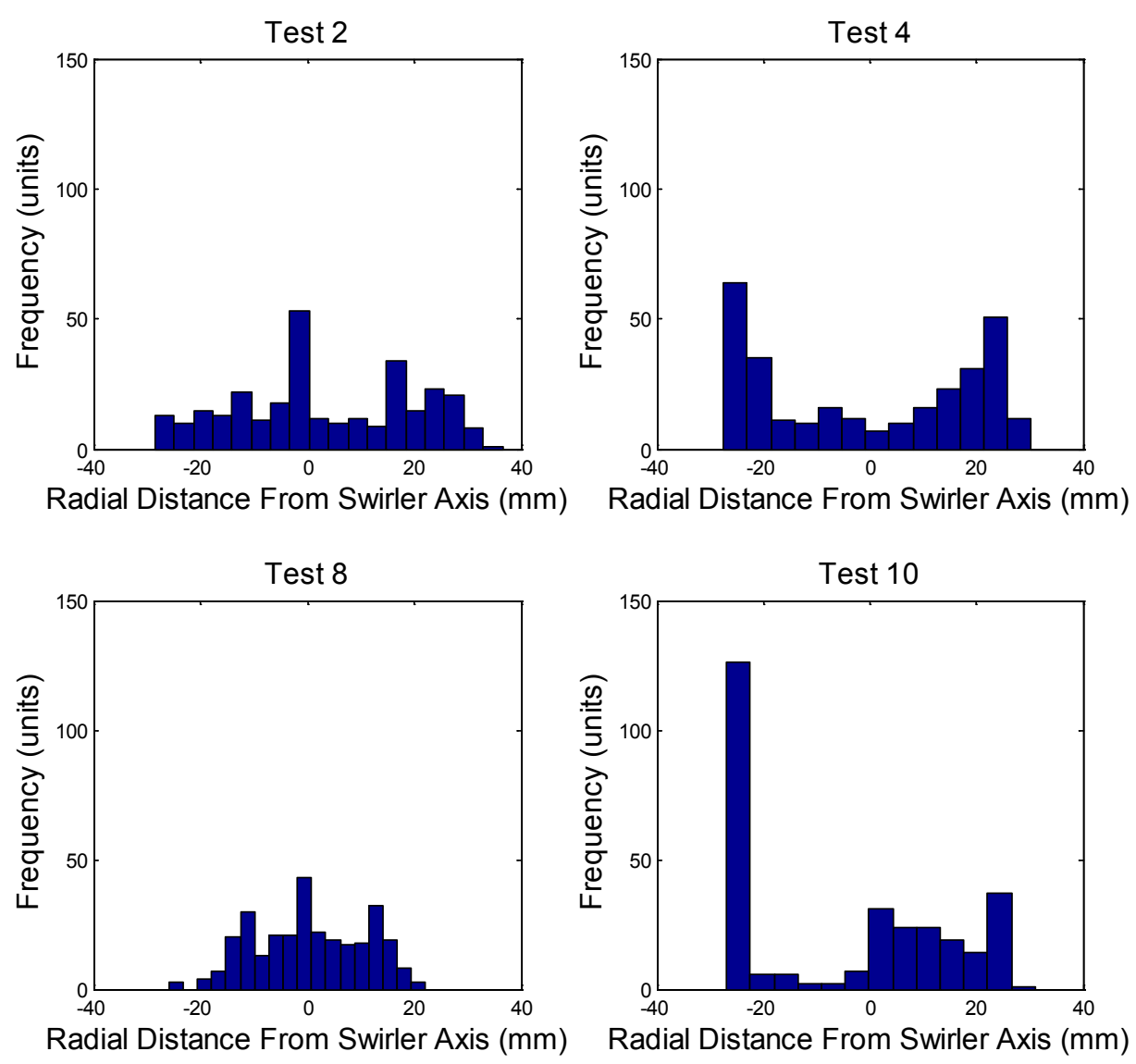

Figure 4.23 Histogram (frequency) of radial flame ignition locations.

\section{Summary}

Three-dimensional PIV techniques were applied to the swirling flow in a model gas turbine combustor to investigate the swirl structure for both non-reacting and reacting flows. Combined images of flame chemiluminescence $\left(\mathrm{CH}^{*}\right)$ and flow field were gathered for the reacting flow conditions. The ambient pressure methane-air combustor operated at constant air airflow rate of $150 \mathrm{slm}$, with changes in methane flow control the degree of lean combustion. In the non-reacting case, a helium-nitrogen mixture provides a surrogate for the methane fuel. Visual observation of the reacting zone was carried out to identify the flame behaviors. A comparison of the swirling flow structures between 
the short and long residence flame bases were made to understand the flame ignition process affected by the swirling flow.

The results showed the central recirculation zone (CRZ) were distinctively captured in the non-reacting flow while the CRZ zone were not resolved for the reacting flow. The influence of the equivalence ratio on the flame stabilization was more pronounced compared to that affected by the degree of mixing. The flames studied showed the combined effects of the partially premixed and diffusion modes, which significantly affect flame dynamics in terms of flame ignition. In the current flame conditions, a high equivalence ratio provided a condition for stable flame anchoring. In terms of swirler residence time, the long residence swirler relatively provided the better flame anchoring. This is consistent with the flow field structure as observed in the velocity vector field, showing a smaller inner recirculation zone in the long residence swirler. The mixing of fresh reactants and combustion products presented in the IRZ promotes combustion rate, causing relatively radial spread burning. Homogeneity of mixing process near the dump plane yields a condition for better burning rate.

\subsection{Characterization of the AERB Combustion Vessel}

\section{Objective}

The objective of this test was to make PIV measurements of a strong three dimensional flow using a two-component PIV measurement configuration on a closed system, to aid in characterization of fan driven mixing inside a closed combustion vessel.

\section{Motivation}

The measurement of turbulent mixing flow inside an optical constant volume combustion chamber was the third application of particle image velocimetry. Two component planar PIV was used to characterize the turbulent mixing flow induced by two fans inside the combustion chamber. 
The optically accessible constant volume combustion vessel, operated by Michigan Technological University's Advanced Internal Combustion Engines (AICE) group in the Alternative Fuels Combustion Laboratory (AFCL) is used to facilitate the need for laboratory testing and development in the understanding of combustion fundamentals. To do this, the combustion vessel is used to aid in a variety of studies on spray dynamics, ignition and flame kernel development, lean condition ignition and combustion properties, laminar and turbulent flame propagation, and emissions characterization for both conventional fuels as well as alternative and multi-component fuels. These studies aid in the development of both gasoline and diesel engine based combustion strategies.

For laboratory studies to be implemented in improved engine design, establishment of the correlation between the conditions inside the combustion vessel and those in an engine cylinder is critical. In order to simulate the environment seen in an engine cylinder, the combustion vessel is capable of both high temperature and pressure, while also being equipped with fans capable of simulating the turbulent flow characteristics experienced in an engine cylinder. This study focused on characterizing the flow structure produced by the mixing fans at a series of operating levels inside the combustion vessel. The PIV measurements allowed for quantitative as well as qualitative investigation of flow structures including the mean velocity fields as well as turbulent velocity fields for correlation to in-cylinder combustion conditions.

Two component planar PIV was used due to the limited viewing angles, making a stereo PIV configuration infeasible. This presented a challenge, as the flow inside the combustion chamber resulting from the mixing fans has a strong three dimensional nature, introducing a strong out of plane velocity that was not captured by the PIV measurement.

\section{Hardware Setup}

The combustion vessel has a 1.1 liter internal volume whose geometry is created by the intersection of three 4 inch diameter cylinders. The six sides of the vessel serve as windows used for instrumentation of the vessel with a variety of components including 
combinations of fans, spark plugs, and fuel injectors or to provide optical access facilitating a variety of optical measurements including shadowgraph imaging, schlieren imaging, Rayleigh light scatter imaging, chemiluminescence, particle image velocimetry, or other optical and laser based diagnostics measurement techniques. In addition to the six windows, the combustion vessel contains eight ports allowing for a variety of sensor instrumentation and flow control. A diagram and image of the combustion vessel housed in the AFCL is shown in Figure 4.24.

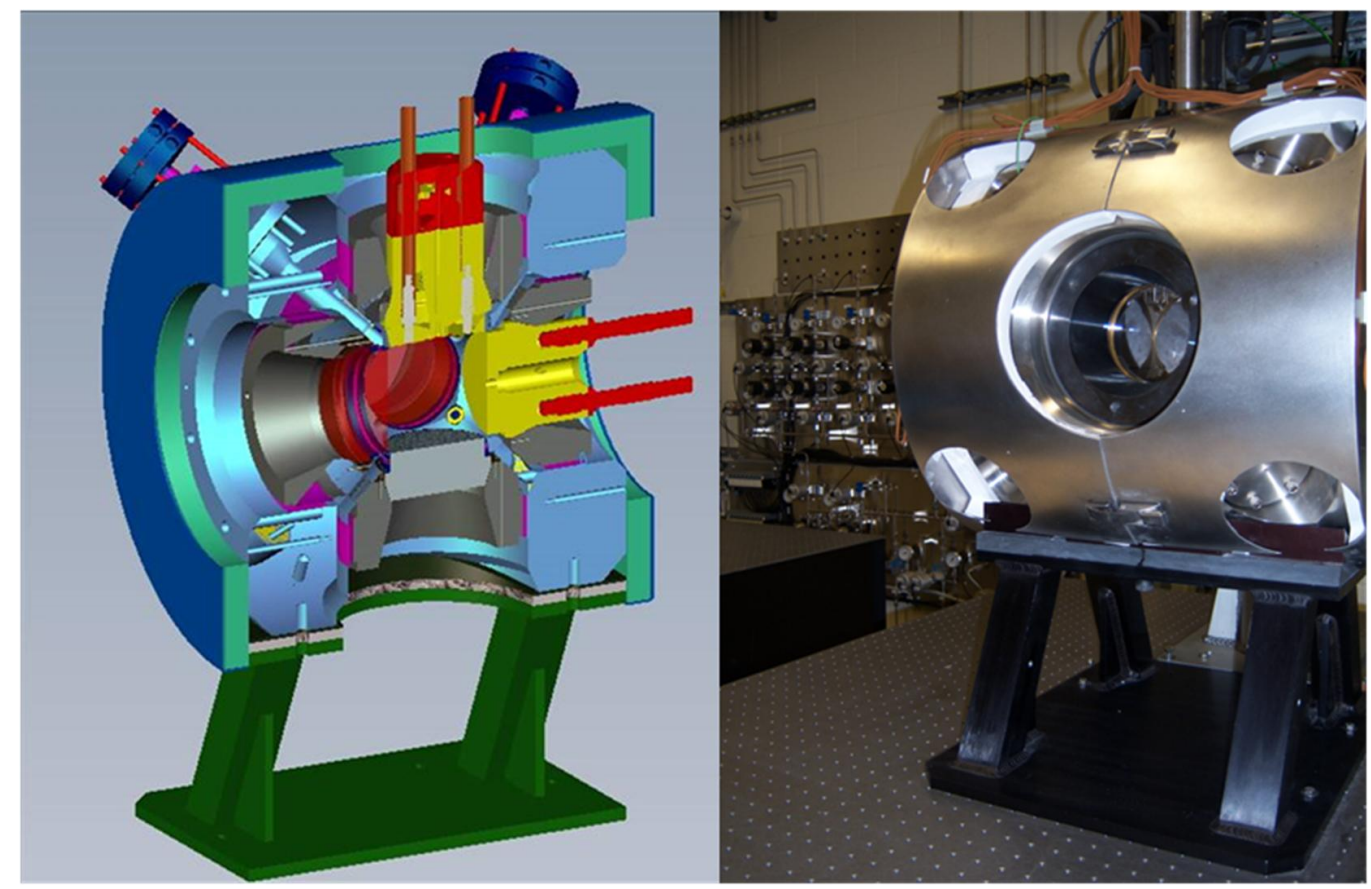

Figure 4.24 CAD model of the constant volume combustion vessel (left) as in stalled in the AFRC (right).

This study focused on characterization of the flow created by a window inserted into the top port of the combustion vessel to hold an assembly of two mixing fans and a spark plug holder. The fans used in this assembly were an eight blade, constant attack angle $\left(30^{\circ}\right)$ design with a 1.0 inch diameter and 0.25 inch depth at the radial edge and blade thickness of 0.035 inch. Full details of the fan dimensions are given in the detailed fan drawings of Figure 4.25. 


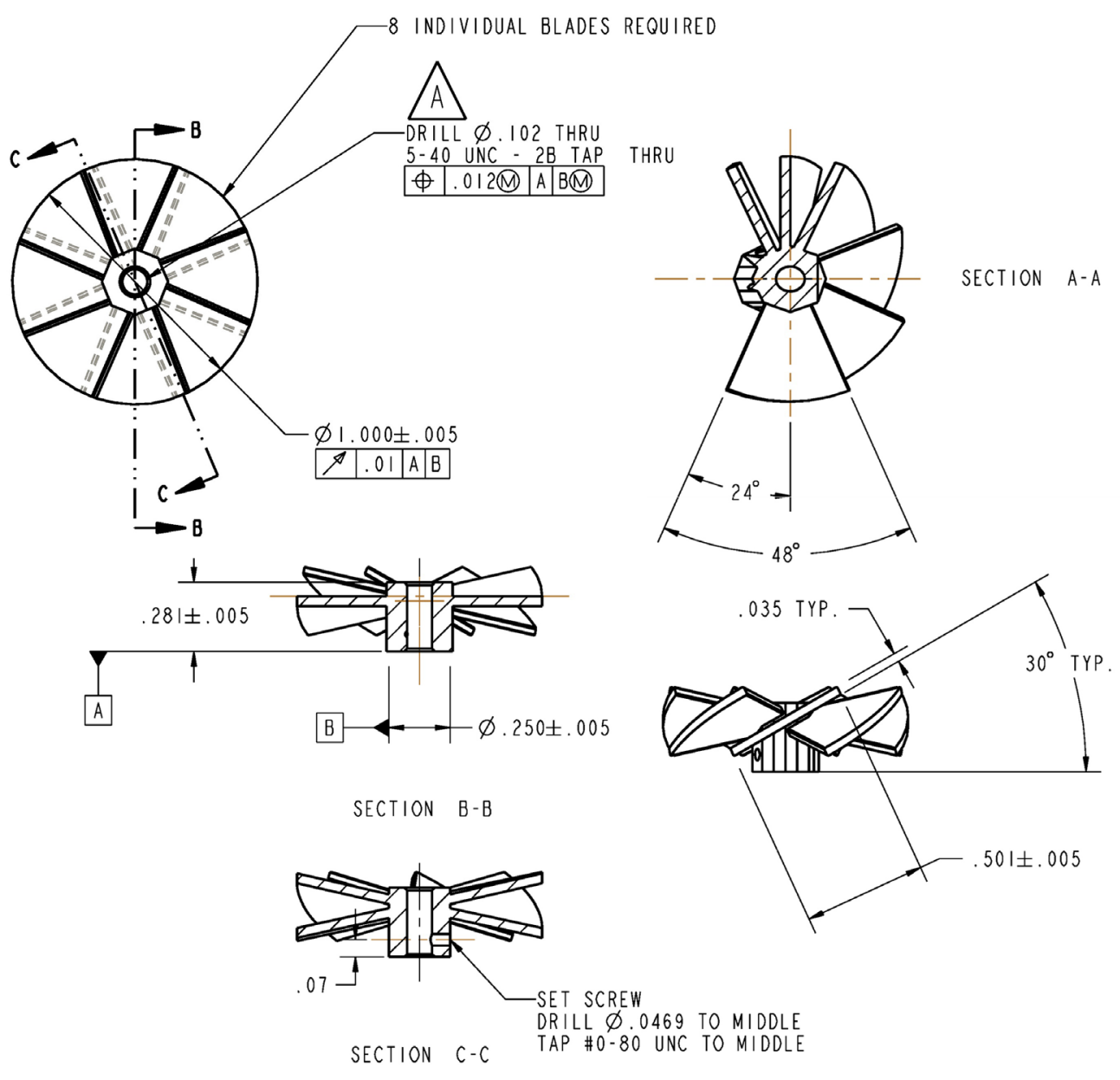

Figure 4.25 Detailed drawings of combustion vessel fan blades.

The positions of the fans and spark plug holder in the window assembly are shown in Figure 4.26. During testing, the spark plug (green) was backed off so that the ground electrode was flush with the spark plug holder (dark red) to avoid reflections of the laser off the electrode and surrounding ceramic. To avoid harsh reflections of the shiny metallic surfaces, the spark plug holder and fan assemblies were coated in a thin layer of flat black paint to minimize and diffuse and light reflections during PIV measurements. 


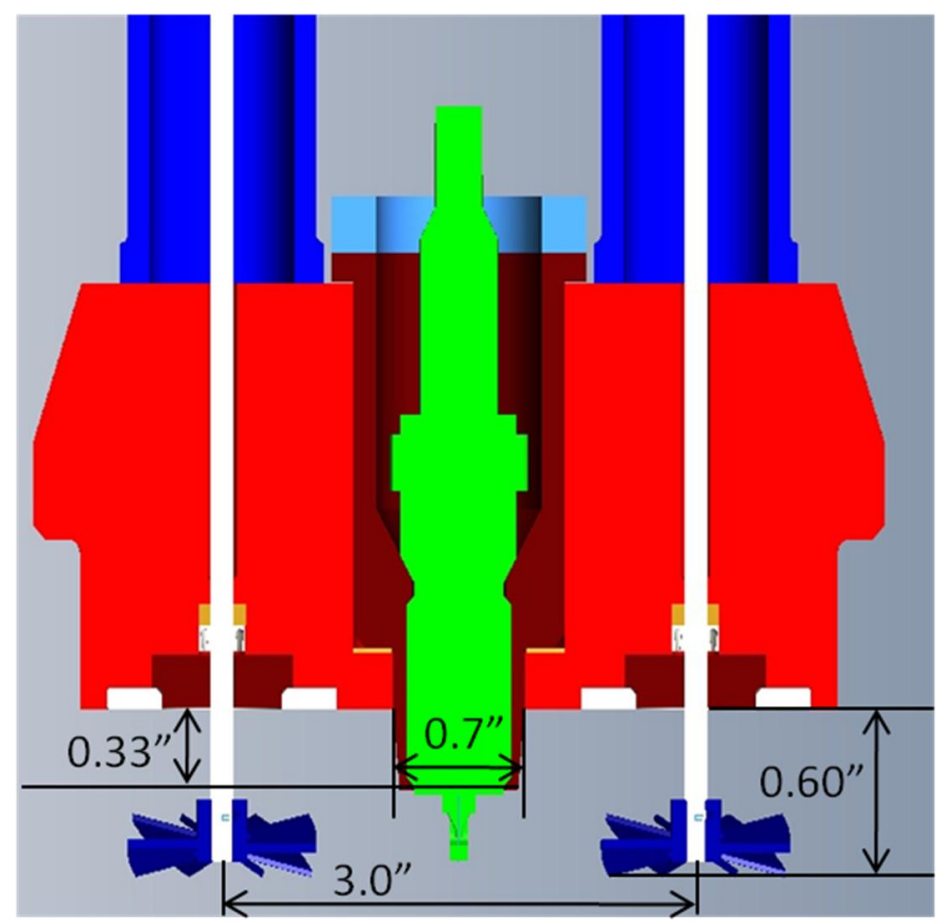

Figure 4.26 Fan blade and spark plug holder positions within the combustion vessel.

Due to limitations in optical access, the fan speeds could not be measured with all the optical windows installed in the combustion vessel, therefore, a supply voltage to fan speed correlation was established prior to the PIV testing, allowing for the fan speed to be set during PIV testing by setting the supply voltage to the fans externally. The fan system is a fairly simple setup, consisting of a single or bi-directional AC motor, a rheostat speed controller, a flex shaft, three-jaw chuck, rigid shaft, and the fan blade assembly. To measure the fan speed, the rigid shaft, which passes through the fan holder located in the top optical port on the combustion vessel, was lowered slightly to provide an approximate 3/8" gap between the fan blade assembly and the combustion chamber ceiling. A piece of reflective tape was placed on the shaft in this gap to allow use of a laser tachometer to measure the shaft speed in revolutions per minute. The output AC voltage was then monitored using a Fluke model 87 II digital multimeter. Care was taken to ensure that the flex shafts for the fan motors were in a neutral location where they 
would remain without binding due to the fact that change in the flex shaft angles can result in more or less resistance in the shaft changing the fan voltage-rpm relationship. The fan was then turned on and the speed was increased incrementally from zero to over $8000 \mathrm{rpm}$, taking voltage readings at each step. This process was repeated again as the fan speed was decreased back to zero rpm. This procedure was performed for the single direction motor, and both forward and reverse directions on the bi-directional motor.

\section{PIV Setup}

For this series of tests individual optical lenses were used to produce a light sheet, and therefore measurement plane, that passed through the combustion vessel bisecting the combustion chamber vertically with the spark plug electrode captured in the measurement plane. Although a light sheet configuration using three cylindrical lenses to produce a non-diverging collimated light sheet would have been preferable for measurements inside the combustion vessel, only one cylindrical lens was available for light sheet formation. Therefore, the light sheet was configured using a cylindrical lens and $500 \mathrm{~mm}$ focal length spherical lens to produce a light sheet with minimal divergence in width to reduce reflections inside the combustion vessel and a nominal sheet thickness

of $1 \mathrm{~mm}$. The window assembly holding the fans and spark plug holder was oriented to position one fan in front of the measurement plane and one fan behind the measurement plane with equal spacing as shown in Figure 4.27.

Seeding of the flow inside the combustion vessel was provided by the single nozzle seeding unit outlined in the first PIV test application. The seeder was directly connected to the intake port on the combustion vessel, located in the upper corner of the chamber volume adjacent to the exhaust port. Flow from the seeder was provided by the building's compressed air supply. 


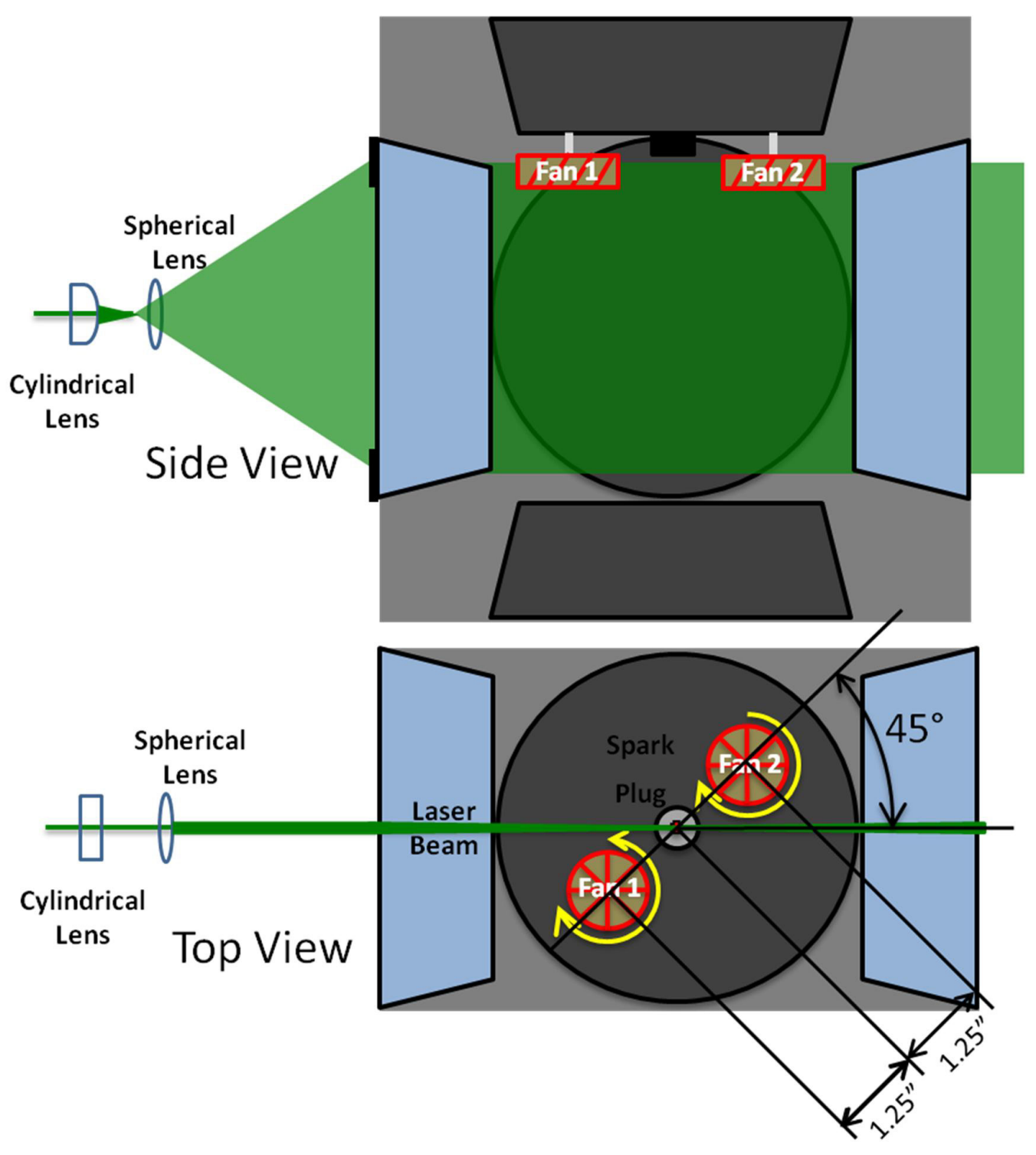

Figure 4.27 Light sheet orientation inside combustion vessel.

The PIV camera was oriented orthogonal to the light sheet with a field of view measuring $39.3 \mathrm{~mm}$ by $30 \mathrm{~mm}$. The camera's field of view was centered on the spark plug holder with the spark plug holder bordering the top edge of the measurement region. A camera aperture f-number of 5.6 was used for all tests. 


\section{Test Conditions}

Each test condition was executed by first opening both the intake and exhaust valves to the combustion vessel. The fans were then set to the desired speed and direction for the test. The valves on the seeder were then opened to supply air to the laskin nozzle generating DEHS oil seeding particles in addition to a small flow through the bypass valve to carry the particles into the combustion vessel. With the small size of the combustion chamber and no primary flow, the combustion volume was rapidly saturated with seeding particles homogenously distributed by the fan motion. At this point, the two seeder valves were closed, ceasing flow into and out of the combustion vessel. The PIV system was then operated to make real time measurements of the flow. Once imaging started to take place, the bypass valve on the seeder was opened slightly to supply additional air to the combustion chamber. Mixed with the seeded flow, the seeding density was slowly reduced until a desirable concentration was achieved for PIV measurements. Once the desired seeding concentration was achieved, the intake and exhaust ports on the combustion vessel were closed. The time interval between image frames was then incrementally increased until the loss of particle image pairs between image frames started to result in invalid measurements. The time interval was then decreased slightly and the PIV system restarted for data collection. The setup procedure provided approximately five minutes for fan mixing in the combustion chamber to stabilize before final PIV measurements were made. For each test, the laser was operated at an energy level of $50 \mathrm{~mJ}$ per pulse, with a series of 200 images taken.

The flow structure inside the combustion vessel, near the spark plug region, was studied for co-rotating and counter rotating fan configurations, where co-rotating fans refers to both fans moving in a clockwise motion as view from above the combustion chamber. In the counter-rotating cases, the fan reversible fan, positioned between the PIV camera and the measurement plane was set to reverse resulting in counter-clockwise rotation of the fan blades as viewed from above the combustion vessel while the single direction fan remained on in a clockwise rotation. Testing was performed at ambient temperature and pressure as well as with an elevated temperature of $100{ }^{\circ} \mathrm{C}$. The elevated $\mathrm{CV}$ temperature 
resulted in the DEHS particles evaporating to the point of insufficient seeding density for accurate PIV measurement over a period of $\sim 75$ images or 1.2 minutes. Consistent seeding was established by opening both the intake and exhaust valves and slowly adding seeded air. The flow rate from the seeder was low enough that it did not appear to influence the flow structure in the combustion vessel produced by the mixing fans, since they were operating at a high speed. However, it may have had a cooling effect on the air charge in the combustion vessel. The conditions for each flow test are shown the test matrix of Table 4.2.

Table 4.2

Test matrix for combustion vessel measurements.

\begin{tabular}{|c|c|c|c|c|c|c|c|c|}
\hline $\begin{array}{c}\text { Test } \\
\text { \# }\end{array}$ & $\begin{array}{c}\text { Fan } \\
\text { Speed }\end{array}$ & $\begin{array}{c}\text { Fan } \\
\text { Rotation } \\
\text { Direction }\end{array}$ & $\begin{array}{c}\text { Pulse } \\
\text { Time }\end{array}$ & $\begin{array}{c}\text { Vessel } \\
\text { Temperature }\end{array}$ & $\begin{array}{c}\text { Selectable } \\
\text { Fan Target } \\
\text { Voltage }\end{array}$ & $\begin{array}{c}\text { Set } \\
\text { Voltage }\end{array}$ & $\begin{array}{c}\text { Single } \\
\text { Direction } \\
\text { Fan Target } \\
\text { Voltage }\end{array}$ & Set Voltage \\
\hline & (RPM) & $(-)$ & $($ us $)$ & $\left({ }^{\circ} \mathrm{C}\right)$ & $(\mathrm{v})$ & $(\mathrm{v})$ & $(\mathrm{v})$ & $(\mathrm{v})$ \\
\hline 10 & 0 & OFF & 75000 & amb./15 & 0.00 & NA & 0.00 & NA \\
\hline 11 & 2000 & Co-Rotating & 2500 & amb./15 & 30.80 & $30.96-31.13$ & 15.19 & $15.25-15.60$ \\
\hline 12 & 4000 & Co-Rotating & 1000 & amb./15 & 42.01 & 42.05 & 18.10 & 18.13 \\
\hline 13 & 6000 & Co-Rotating & 600 & amb./15 & 49.40 & $49.65-49.81$ & 21.15 & $21.25-21.35$ \\
\hline 14 & 8000 & Co-Rotating & 400 & amb./15 & 55.47 & $55.35-55.83$ & 23.76 & $23.80-23.95$ \\
\hline 15 & 2000 & $\begin{array}{c}\text { Counter- } \\
\text { Rotating }\end{array}$ & 500 & amb./15 & 32.45 & $37.39-32.94$ & 15.19 & $15.19-14.55$ \\
\hline 16 & 4000 & $\begin{array}{c}\text { Counter- } \\
\text { Rotating }\end{array}$ & 200 & amb./15 & 44.74 & $44.54-44.43$ & 18.10 & $18.26-18.83$ \\
\hline 17 & 6000 & $\begin{array}{l}\text { Counter- } \\
\text { Rotating }\end{array}$ & 100 & amb./15 & 51.29 & $51.44-51.48$ & 21.15 & $21.20-21.04$ \\
\hline 18 & 8000 & $\begin{array}{l}\text { Counter- } \\
\text { Rotating }\end{array}$ & 50 & amb./15 & 57.71 & $57.65-57.22$ & 23.76 & $23.65-23.30$ \\
\hline 19 & 8000 & FWD/Off & 100 & amb./15 & 55.47 & 55.4 & 0.00 & 0 \\
\hline 20 & 8000 & REV/Off & 100 & amb./15 & 57.71 & $57.80-57.75$ & 0.00 & 0 \\
\hline 22 & 0 & Off & 10000 & 100 & 0.00 & 0 & 0.00 & 0 \\
\hline 24 & 8000 & Co-Rotating & 300 & 100 & 55.47 & $55.45-55.35$ & 23.76 & $23.80-23.51$ \\
\hline 26 & 8000 & $\begin{array}{c}\text { Counter- } \\
\text { Rotating }\end{array}$ & 100 & 100 & 57.71 & $57.70-57.60$ & 23.76 & $23.81-24.65$ \\
\hline
\end{tabular}


In the case of quiescent flow, the fans were utilized to establish a homogenous mixture during seeding of the combustion vessel, after which the fans were turned off and the flow was allowed to subside over a 10 minute period prior to making flow field measurements.

\section{Results and Discussion}

The resultant calibration data was then entered into MatLab to produce the interactive calibration curves seen in Figure 4.28, Figure 4.29, and Figure 4.30.

Comparison of the single direction motor calibration curve to the bi-directional motor calibration curves shows a stark difference in the voltage-rpm relationship, achieving similar revolutions per minute as less than half the voltage. The forward and reverse rotational directions on the bi-directional motor also indicated different voltage-rpm relationships, necessitating all three calibration curves to be developed. Comparison of the calibration curves also shows lower variability in the single direction motor compared to the bi-directional motor for both forward and reverse directions.

Since the flow inside a combustion cylinder is more volatile than that which can be produced by the mixing fans in the lab's combustion chamber, the flow cases at which the fans are operating at maximum speed are of greatest interest. The mean flow field velocity profiles for the ambient temperature condition cases of no fan mixing and 8000 rpm co-rotating counter-rotating and single fan mixing are shown in. The velocity profiles have been overlaid on an image of the combustion vessel as viewed by the PIV camera, showing the reversible mixing fan in front of the measurement plane and to the left of the spark plug while the single direction fan is on the right and behind the measurement plane. As previously mentioned, the light sheet was centered on the spark

plug electrode with the spark plug threaded up into the spark plug holder, the edge of which can be seen in the background image, to avoid reflections during the series of PIV measurements. The spark plug was used in the background image for reference purposes only. 


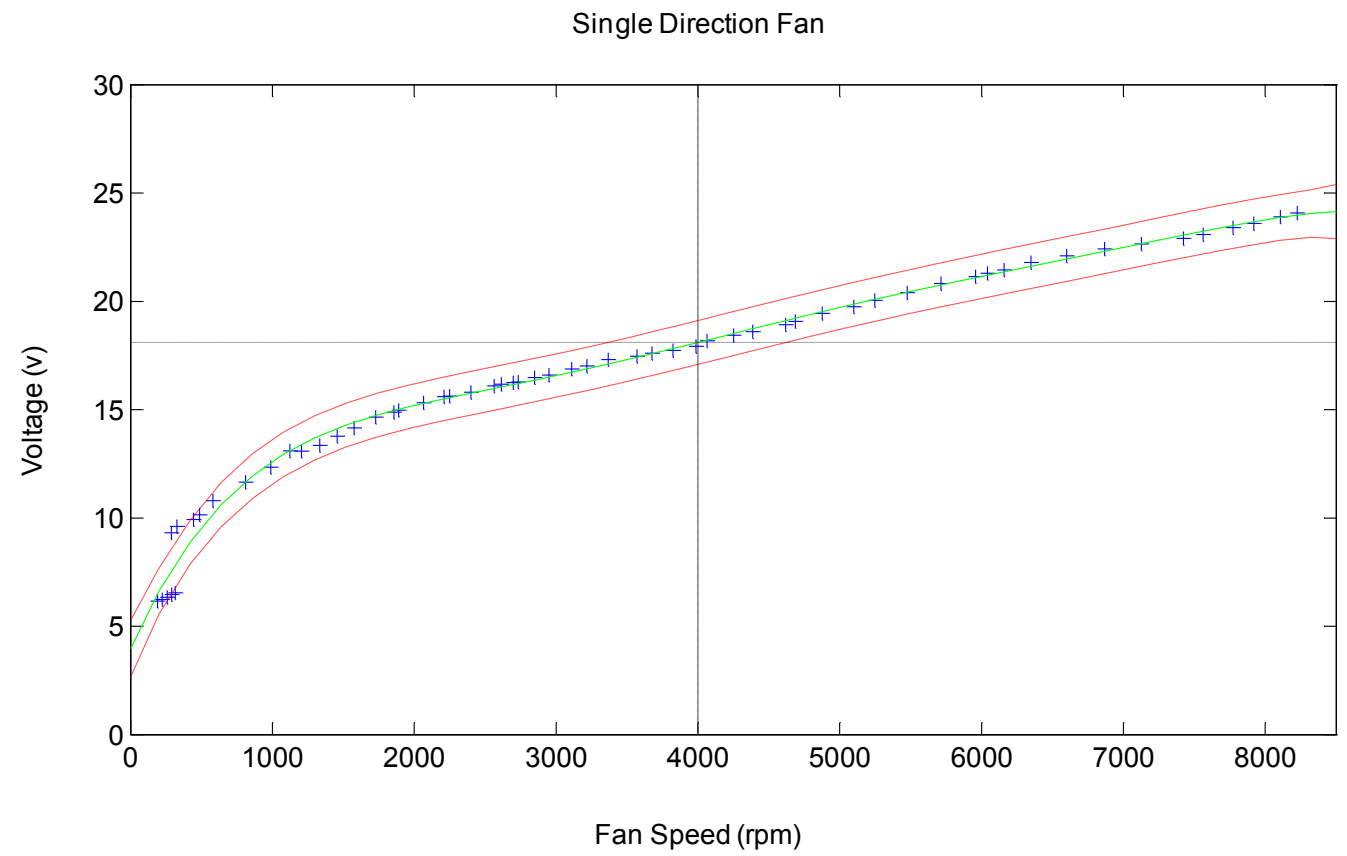

Figure 4.28 MatLab fan calibration plot for single direction fan.

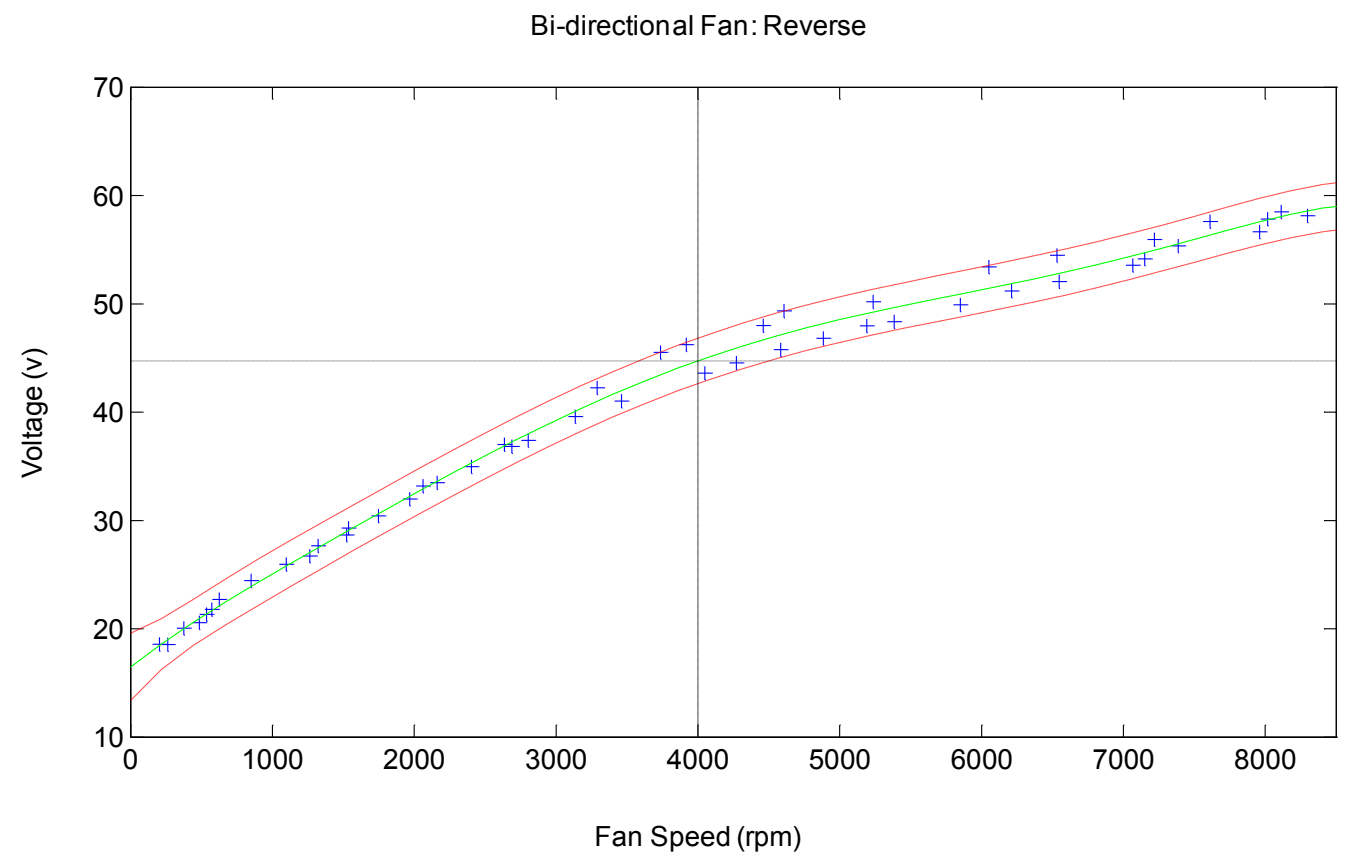

Figure 4.29 MatLab fan calibration plot for bi-directional fan - reverse. 


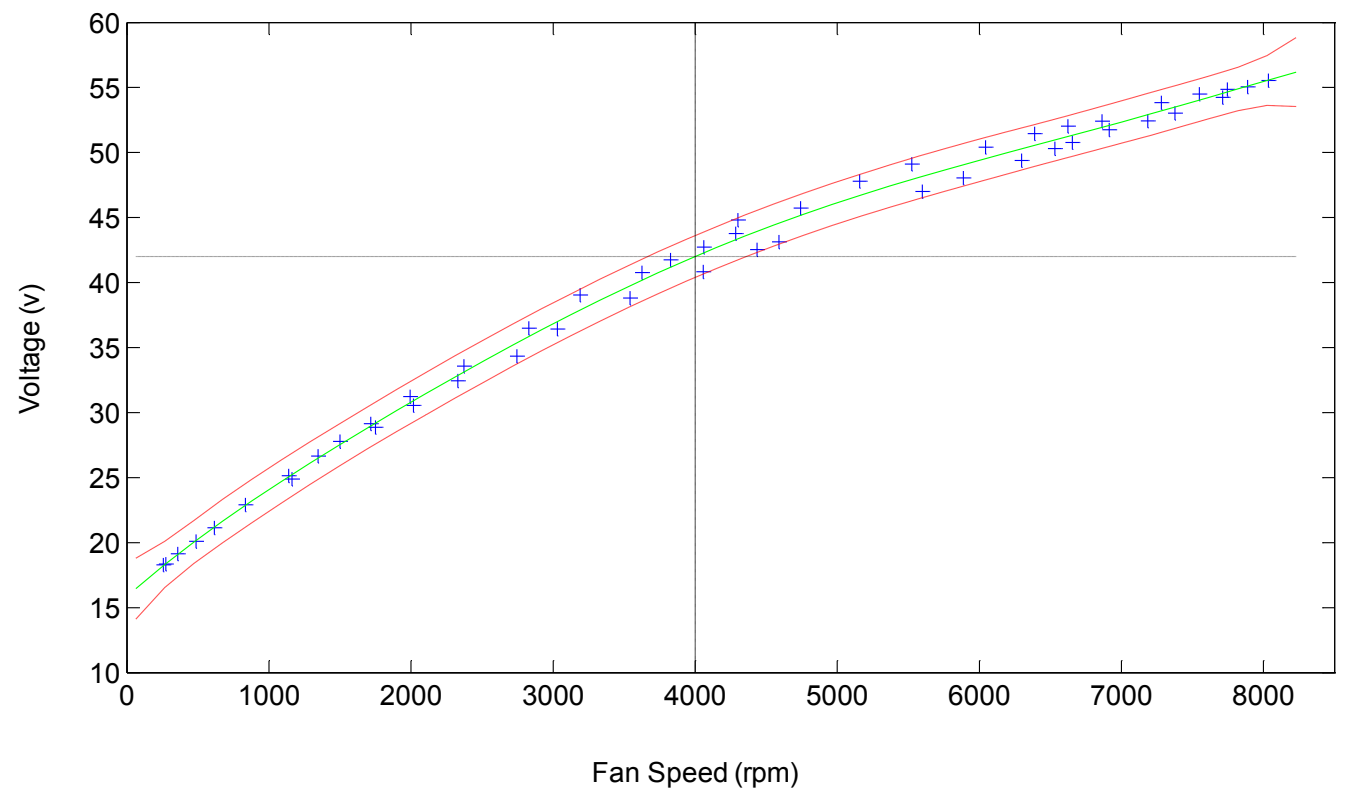

Figure 4.30 MatLab fan calibration plot for bi-directional fan - forward.

In the absence of mixing by the fans, the quiescent flow field shows minimal velocity, on the order of tenths of millimeters per second, as would be expected. For the $8000 \mathrm{rpm}$ fan speed cases shown, the velocity profiles share a similar range in magnitude of 0 to 1 $\mathrm{m} / \mathrm{s}$. In the case of co-rotating fans, flow in the combustion vessel shows a strong upward velocity at the center of the combustion vessel, spanning the measurement region, while flow near the spark plug holder is lower in velocity. This is not surprising, as the top of the fan assemblies are about flush with the spark plug holder. This shields the region where a spark plug would be from the flow, and with flow going in the same direction for both fans, there is minimal cross flow seen. In contrast, with the fans operating under counter-rotating conditions, where the left fan is pushing air down while the right fan pulls air up, a significant cross flow velocity in the spark plug region is observed. The same trend is exhibited in the case of the flow being driven by just the left fan pushing air down. In both of these cases the flow velocity between the fans and near to the spark plug is greater than the average velocity for the measurement region as a whole. The mean velocity profiles for each test condition are provided in the attached appendix. 
The mean velocity profiles show part of the flow characteristics, the non-steady state component of the flow, the turbulent flow, also provides insight into the flow conditions in the combustion vessel. The mean turbulent velocity profiles shown in Figure 4.32 where calculated by applying the following equation to the series of PIV measurements for each test:

$$
\text { Mean Turbulent Velocity }=\left(\frac{1}{n-1} \sum_{i=1}^{n}\left(v_{i}-\bar{v}\right)^{2}\right)^{\frac{1}{2}}
$$

In the equation, $v_{i}$ is the instantaneous velocity magnitude derived from a given interrogation region in the PIV measurement and $\bar{v}$ is the average velocity magnitude at the same interrogation region for all measurements in the test. The contour plots of mean turbulent velocity in Figure 4.32 correspond to the three $8000 \mathrm{rpm}$ fan speed test cases plotted in .

Although the co-rotating fans produced a lower mean velocity measurement near the spark plug compared to the surrounding measurement region, Figure 4.32, shows that this region has a higher turbulent velocity compared to its surroundings. Comparison of the turbulent velocity contour plots for the co-rotating and counter-rotating fan cases shows a significant difference in turbulent velocity. While the co-rotating fan configuration produced elevated turbulent velocity between the fans at mid blade height, the flow in the counter-rotating case shows a substantial vertical gradient in turbulent velocity over the entire measurement region. In the case of only the directional fan operating at $8000 \mathrm{rpm}$, pushing air downward, turbulent velocities are observed at slightly higher levels than those seen in the case of co-rotating fan operation and lower than the counter-rotating fan case. In contrast to the dual fan cases, the region of greatest turbulent velocity does not occur between the fans, but in the region below the fans. The complete series of turbulent velocity profiles can be found in the attached appendix. 


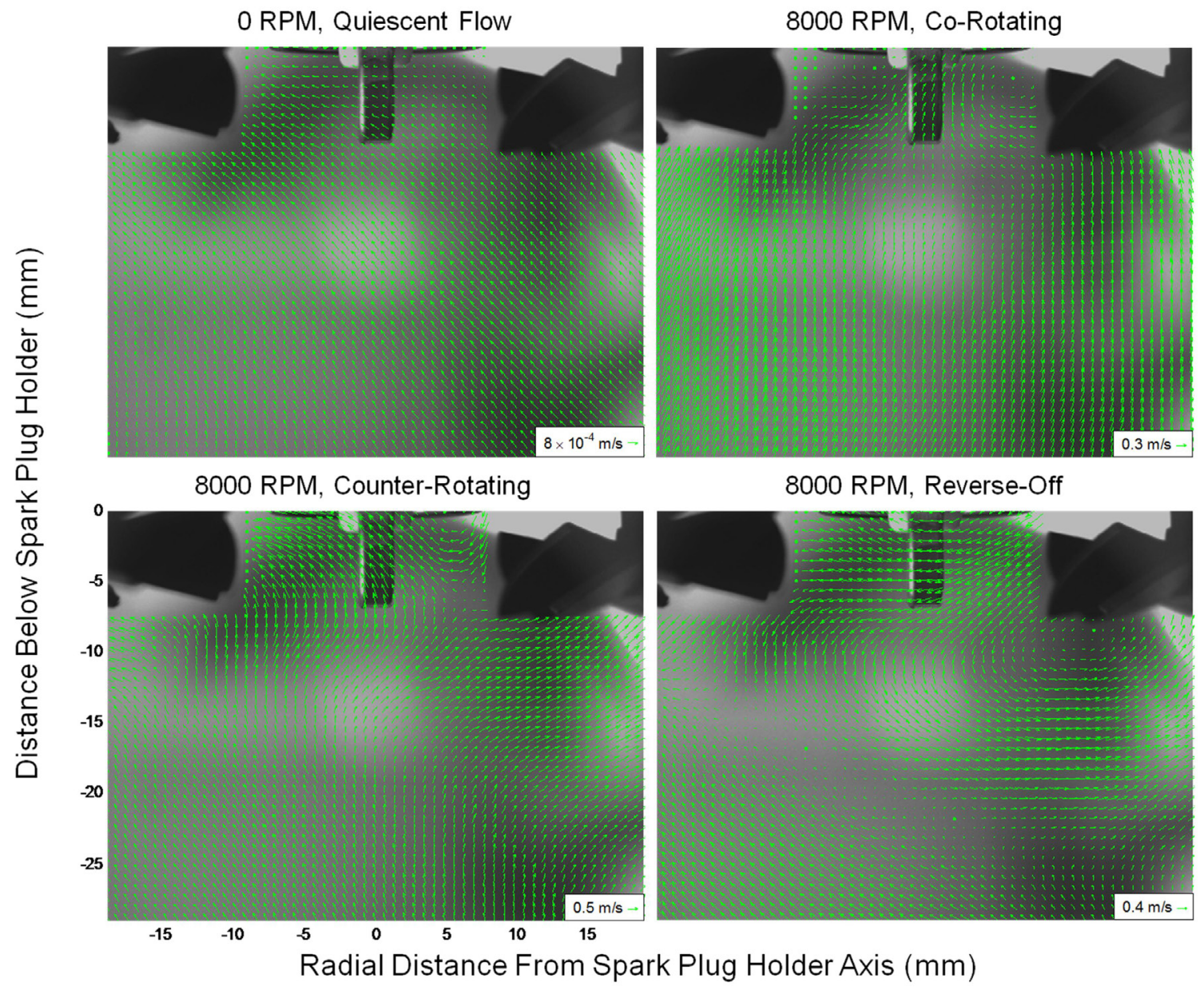

Figure 4.31 Mean velocity profiles near the spark plug for the quiescent flow of Test 10 (top left), and the $8000 \mathrm{rpm}$ fan speed cases of co-rotating fans (top right) counterrotating fans (bottom left) and operation of the bi-directional fan in reverse only (bottom right). 


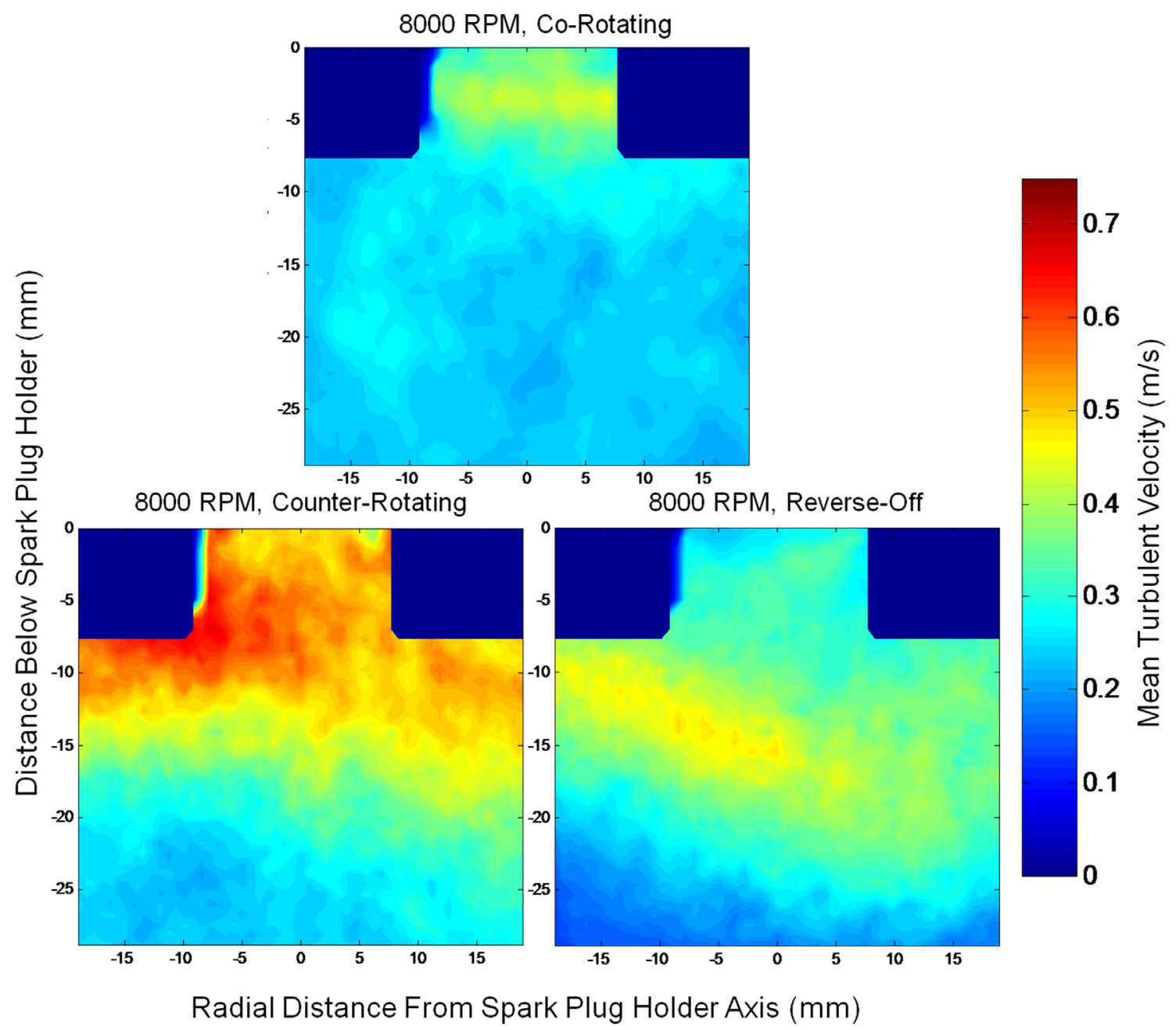

Figure 4.32 Mean turbulent velocity profiles for the co-rotating (top), counter-rotating (bottom left), and single fan $8000 \mathrm{rpm}$ operating speed conditions of Test 14, 18 and 20 respectively. 


\section{Summary}

Establishing a basic understanding of the flow characteristics within the optical combustion chamber is important establishing a relationship between combustion performance seen during laboratory testing and expected results in an application environment. This series of PIV measurements provided a base characterization of the flow produced by the combustion vessel's mixing fans. The $8000 \mathrm{rpm}$ test cases were chosen for review due to their high mixing level being the closest representation of the flow seen inside a combustion cylinder. In addition numerical simulation for these test cases was performed by a third part and shown to have good agreement in both bulk velocity characteristics as well as turbulent velocity characteristics. These experimental results provide a foundation for the validation and development of further numerical simulation to characterize fan flow within the combustion vessel, with the goal of eventual characterization of reacting flow conditions. 


\section{CHAPTER 5: SUMMARY AND FUTURE WORK}

\subsection{Summary}

Planar particle image velocimetry principles and test considerations were investigated through application of both two component and three-component velocity measurement systems in non-reacting reacting and flow. Application of the measurement system provided an increased understanding of the governing principles required to achieve reliable and accurate PIV measurements as well as insight into more intuitive considerations for testing to enhance overall PIV system performance.

The variety of flow measurements conducted in this study showed the versatility of the measurement method's application as well as desirable characteristics in fluid dynamics research through its whole field measurement approach providing quantitative and qualitative characterization of flow fields, the non-intrusive ability to make measurements near boundaries without disturbing the fundamental flow, and the volume of information that can be collected. 


\subsection{Future Work}

The flow measurements yielded from the application of the PIV system in this work will serve primarily as a baseline starting point and background information for future studies concerning the model gas turbine combustor and optical combustion chamber.

Future applications of PIV measurements combined with planar laser induced fluorescence (PLIF) or other laser diagnostic techniques to the model gas turbine combustor could yield an array of in-depth studies on swirl stabilized premixed/prevaporized combustion dynamics, including mixedness, emissions, heat release, and instability in gas combustor dynamics.

The characterization of the fan induced turbulent flow in the combustion vessel served as an initial probe into fluid dynamics of the combustion chamber. A base understanding of the flow motion inside the combustion vessel near the spark plug provides critical background information for the study of spark kernel development, ignition studies, and flame propagation studies. Furthermore, the experimental measurements will aid in more accurate numerical modeling of the combustion vessel and the characteristics for fan induced flow, ideally leading to future modeling of reacting flows inside the combustion vessel. 


\section{REFERENCES}

1. Adrian RJ. Twenty years of particle image velocimetry. Experiments in Fluids 2005;39(2):159-169.

2. Particle Image Velocimetry (PIV) [Internet]. Skovlunde: Dantec Dynamics A/S. 2011 [cited 5-5-2011]. Available from: http://www.dantecdynamics.com/

3. Raffel M, Willert CE, Wereley ST, Kompenhans J. Particle Image Velocimetry A Practical Guide. Berlin Heidelberg: Springer; 2007. 253 p.

4. Particle Image Velocimetry [Internet]. Tamil Nadu, India: ValueBound. 2008 [cited 5-30-2011]. Available from: http://velocimetry.net/principle.htm

5. Keane RD, Adrian RJ. Theory of cross-correlation analysis of PIV images. Applied Scientific Research 1992;49(3):191-215.

6. Hain R, Kähler C. Fundamentals of multiframe particle image velocimetry (PIV). Experiments in Fluids 2007;42(4):575-587.

7. Bouvet N. PDE Project PIV Experiment, Part 1.

8. Inc NWR. Operator's Manual New Wave Research, Inc Tempest and Gemini PIV Nd:YAG Lasers. 200. p 64.

9. C8484-05C [Internet]. Beijing, China: Hamamatsu Corporation. 2011 [cited 4-172011]. Available from: http://sales.hamamatsu.com/en/products/systemdivision/machine-vision/part-c8484-05c.php

10. AF Micro-NIKKOR 60mm f/2.8D [Internet]. Nikon Inc. 2011 [cited 4-17-2011]. Available from: http://www.nikonusa.com/Nikon-Products/Product/CameraLenses/1987/AF-Micro-NIKKOR-60mm-f\%252F2.8D.html\#tab-

ProductDetail.ProductTabs.TechSpecs

11. Huang Y, Yang V. Dynamics and stability of lean-premixed swirl-stabilized combustion. Progress in Energy and Combustion Science 2009;35(4):293-364. 
12. Seo SaL, S.-Y. Effects of Unmixedness on Combustion Instabilities in a LeanPremixed gas Turbine Combustor. Flow turbulence Combustion 2010(85):17.

13. Turns S. An introduction to combustion : concepts and applications. WCB/McGraw-Hill; 2000.

14. Lilley D. Swirl Flows in Combustion: A Review. AIAA Journal 1977;15:15.

15. Mordaunt C, Lee, S.-Y., Kalaskar, V., Mensch, A., Santoro, R., and Schobert, H. Further Studies of Alternative Jet Fuels. 2009.

16. Boxx I, Stöhr M, Carter C, Meier W. Temporally resolved planar measurements of transient phenomena in a partially pre-mixed swirl flame in a gas turbine model combustor. Combustion and Flame 2010;157(8):1510-1525.

17. Sadanandan R, Stohr M, Meier M. Simultaneous OH-PLIF and PIV Measurements in a Gas Turbine Model Combustor. Applied Physics 208;B(90):609-618. 


\section{APPENDIX A: PIV OPERATING PROCEDURES}

\section{2-D PIV Test Procedures}

1. Check that keys in laser power supplies are turned to the "off" position, the laser head aperture is closed, and the cap is in place over the light sheet optic output.

2. Check that lens caps are in place on the camera lenses protecting the filter, lens, and camera CCD from laser light exposure.

3. Ensure the lab door to the room in which the laser is being used is closed, disabling the laser safety interlock.

4. Set the toggle switches for flash lamp and Q-switch control on the back of the laser power supplies to the "int" position and ensure the BNC cables from the FlowManager System HUB laser triggering cable are connected to the "fire" BNC ports.

5. Wearing appropriate $532 \mathrm{~nm}$ laser safety glasses turn both power supply keys to the "on" position. Set both of the laser's manual control box flash lamp dials to the minimum power position. Press the "standby" button on the control boxes to ready the laser for firing.

6. Open the laser head aperture and remove the cap from the light sheet optic.

7. Based on preference, set the laser firing mode to "var", "fixed", or "single shot". With the light sheet optic roughly positioned for proper light sheet positioning, fire the laser, slowly increasing the flash lamp power until the light sheet just barely becomes visible with laser safety glasses in use. *Note, the laser emits nonvisible infrared light even at the minimum flash lamp setting. However, the second harmonic generator used to frequency double the laser light does not activate until a threshold power has been achieved. The IR light emitted prior to visible $532 \mathrm{~nm}$ output is still capable of doing serious damage to the eyes. Do not 
look directly into the laser light sheet, and ensure there are no obstacles through the light sheets path that will reflect a focused beam.

8. Fine tune the light sheet positioning to the desired location on the test article and adjust the light sheet width to meet test condition requirements.

9. Turn the lasers to "standby" and press the "stop" button on the control panel, close the laser aperture and replace the light sheet optic cap.

10. Once the laser is aligned, place the camera in position, perpendicular to the plane of the light sheet. Check that the camera's planned placement is adequate for favorable PIV data collection, as the scale of the image is directly related to the camera's ability to distinguish individual seeded particles in the flow.

11. Adjust camera F-Stop and distance to the model as specified by the tool in step 10.

12. Enter a new calibration setup into the FlowManager software program, ensuring to add a camera 1 image map.

13. Turn on the Flow Map System Hub, once the system hub has booted, initialize the system under the Run tab in the FlowManager software, after initialization of the HUB enter the "Online/Acquisition” mode.

14. With the test article sufficiently illuminated, place a visual reference, preferably a ruler or any object of known dimension in plane with the laser light sheet. This will accomplish three things, it will allow image scaling, focusing of the camera, and field of view evaluation. For additional information regarding image acquisition, refer to the FlowManager Users Guide.

15. Ensure that the LIFO buffer is not selected in the Run tab in the online control window, and initiate the continuous run mode. The system will start to acquire and display camera images. Use this mode to adjust the camera position for an appropriate field of view as well as adjusting the camera lens focus. 
16. Once the camera is positioned and focused, stop the online acquisition, select an appropriate number of bursts, and enter the "Start \& Save" mode to save multiple calibration images.

17. Close the Online/Acquisition interface window.

18. Select a desirable calibration image then go to Setup $\rightarrow$ Field of View $\rightarrow$ Measure, click the "use database" button and place the markers on the calibration image to acquire a scale factor in the results section. Record the scale factor value for use in further test setups utilizing the same physical hardware configuration

19. Once the scale images have been collected, replace the $532 \mathrm{~nm}$ filter and cover on the camera lens. It is important to never activate the laser with the lens or filter off of the camera, as direct or scattered laser light can permanently harm the sensitive CCD sensor.

20. On the power supplies turn the toggle switches for both the flash lamp and Qswitch to the "ext" position, open the laser aperture and remove the light sheet optic cap.

21. Flow the model with $2-5 \%$ of the target airflow and seed the airflow with the desired seeding particles.

a. Turn off the room lights while illuminating the flow field.

b. Collect several seeded images with the laser bright enough to light the particles, but not so bright as to envelop the entire screen in a white region of indeterminable geometry.

c. You should be able to distinguish individual particles and/or any geometric features of the flow. If your geometry causes any shadows, they should be visible here.

d. Save 3-5 images that will be used to create a mask in FlowManager (see user's guide regarding mask creation, purpose and use). 
22. Place a laser Joule meter between the laser head and base of the light guide arm. Enter the Online/Acquisition mode in FlowManager and set the acquisition mode to continuous. Place one of the power supply toggle switches to "int" mode so that only one lasing cavity is active. Set the laser pulse energy to the desired level. Repeat with the laser supply flash lamp toggle switch positions reversed to match the pulse energy of the second lasing cavity with the first. Stop the acquisition through the FlowManager software, remove the Joule meter and return the toggle switches to the "ext" position.

23. With the mask images are collected, you are prepared to take data. Bring the flow up to the target flow conditions as described in the Airflow Testing Work Instructions. Archive your flow conditions with a suitable Data Acquisition unit.

24. When steady state flow is achieved, activate the seeding generator(s) and allow them achieve steady state output as well.

25. Create a new "Setup" for data collection with the appropriate number of bursts and laser pulse interval timing. See the FlowManager User's Guide for information related to manipulation of the burst timing variables. Note: the tool mentioned in step 10 will calculate the MAXIMUM burst timing allowable to collect accurate PIV data (rig specific).

26. When the flow and seeding generator(s) have achieved steady state, activate the FlowManager online acquisition to begin recording PIV images. DO NOT LOOK DIRECTLY INTO THE LASER LIGHT SHEET.

27. If a large number of images or short interval between burst is desired, utilize the LIFO buffer in the "Online/Acquisition" window and select the "update database between bursts" option. Caution must be taken to prevent an overload of the System Hub buffer. This will result in freezing of the system hub requiring a restart. 
28. When an image set has been completed, right click on an image and select "New Dataset". Perform an adaptive correlation on the image to verify that settings are adequate to obtain good measurements. See the FlowManager User's Guide for a full explanation of the many Adaptive Correlation settings and options. However, in general:

e. Use a Gaussian band-pass filter to widen the correlation peaks of particles that may have been focused too well to be distinguished by the camera's CCD chip, leave " $k$ " as the default value

f. Always use the central difference biasing scheme, it is more accurate

g. Use deforming windows

h. If you like: perform peak validation and/or moving average filtering (these help eliminate invalid vectors brought about by incidental correlations of unrelated particles)

29. If the vector map appears valid and no other test will be performed for the current hardware setup/light sheet location, power down the lasers and turn off the seeding generator(s). Open the lab door to activate the laser interlock.

30. If the data does not appear valid, adjust the burst timing and/or interrogation window size and repeat the data collection.

31. Generally an error on the short side of the ideal burst timing is more forgiving than a burst timing that is too long. The shorter burst timing can be counteracted by reducing the interrogation window size. This is preferable to increasing the interrogation window size, as it does not sacrifice resolution.

32. If data collection is finished for the day, cease the airflow to the model as described in the Airflow Work instructions. If not, repeat steps 25-30 for the next test. 
33. Once the laser is powered down, close the laser head aperture, replace the light sheet optic cap, and camera lens covers to prevent dust buildup and protect the optics.

34. Right click on the first image in the Setup file, choose "Select Similar Dataset Records". Right click the image again and choose "New Dataset", then choose the "Analysis Sequence" Tab and generate a new analysis sequence. First a masking operation, selecting the image mask created using images from Step 22 as a "fixed input". Second, set up the same adaptive correlation that resulted in a good data set in Step 28. Finally add a range validation or averaging filter as desired. Select " $O k$ " to apply the analysis sequence to all selected image pairs. This step will take some time, especially if you have collected a large number of images and a computationally intensive correlation procedure.

35. When FlowManager has finished processing the data, right click on the desired level of image analysis, again choose the "Select Similar Dataset Records" and choose the "Vector Statistics" processing option under the "Statistics" category in the "Analysis Methods" Tab.

36. Select the result of the statistics package and perform any additional range or filter validation.

37. Repeat steps 33-35 for set of images and test conditions, changing the analysis parameters as appropriate. 


\section{Stereo PIV Test Procedures}

1. Mount two camera and lens-filter-cap assemblies on the Dantec Dynamics stereo PIV mounts.

2. Follow steps 1-11 in the 2-D component procedure.

3. Create a new Setup in FlowManager, adding a second camera and corresponding image map.

4. Turn on the Flow Map System Hub, once the system hub has booted, initialize the system in the FlowManager software, then enter the Online/Acquisition mode.

5. Place a suitable calibration target in plane with the laser light sheet with the face of the target centered in the light sheet.

6. With the test article sufficiently illuminated, acquire images from both cameras in continuous mode. Use the stereo mount to adjust the camera angle to maximize the common field of view for both cameras. Then adjust the lens to camera angle to focus the image over the entire calibration target.

7. Once both cameras fields of view are aligned and the entire measurement plane is in focus, with the target sufficiently illuminated, take several images of the calibration plate from each camera at multiple target positions normal to the light plane (along the Z-axis). A minimum of three positions is required for a linear calibration. This will accomplish three things, it provides a scaling profile for each image accounting for perspective, allows the image to be de-warped if viewing through a curved window, and provides a known relation between both camera view points for combining both camera's 2-D component PIV profiles into a single 3-D component profile.

8. These calibration images can be exported for simple image processing to increase contrast in the dot matrix in a simple program such as Microsoft Office Picture Manager. 
9. Once image processing has been achieved, import the images back into the Flow Manager Environment.

10. For each calibration image, right click on the image and go to properties. In the Log Text comment box record the $\mathrm{Z}$ axis position (in $\mathrm{mm}$ ) of the calibration target corresponding to that image (i.e. $z=-3$ ).

11. Export the Setup in Flow Manager as a setup index file or .six

12. Open the stereo PIV software package FlowManager 3D-PIV, under Calibration Archive add a calibration set with an applicable name. Then click add calibration images.

13. Select the applicable calibration target used or add a custom target and select the camera perspective of the target applicable to the hardware setup.

14. Click " $O K$ ” and select the .six index file created in step 11.

15. Select only the final calibration images to be imported from the .six file. When the images are added, check that there are two images for each $\mathrm{Z}$ position corresponding to each camera.

16. Click on the "Calculate Calibration" button and select a suitable calibration file name and location.

17. If the calibration is calculated successfully, click on one of the calibration image sets. Right click on the target images and select "show calibration". Select the calibration model that was just created. This will show you the field of view shared by the two cameras as well as the marker location and perspective adjustments for the image.

18. If the calibration is unsuccessful, repeat steps 5-16, trying to maximize the overlap between the camera's fields of view and adjusting the lighting for good target contrast. The calibration tolerances and parameters can also be adjusted as recommended in further detail in the 3D PIV Manual 
19. If the results of step 17 are suitable, return the FlowManager software and follow steps $19-33$ in the 2-D component PIV procedure.

20. Once the images have been collected and processed into the desired 2-D component velocity maps, export the setup file as a .six index file.

21. In the FlowManager 3D-PIV software add a new vector set with an appropriate name.

22. Under the new vector set add a set of $2 \mathrm{D}$ vector maps, selecting the .six file created in step 20 .

23. Select the desired level of processed vector maps as well as select individual images if desired.

24. Select "Calculate 3D Vector Maps", and select the applicable calibration set to use.

25. Once the $3 \mathrm{D}$ vector maps have been generated, right click on the " $3 D$ Vector Map" folder and select "Calculate statistic".

26. The FlowManager 3D-PIV options for viewing and processing of 3 component vector maps is quite limited, therefore it is best to export the $3 D$ Vector maps folder for further processing or manipulation in MatLab or Tecplot. 


\section{APPENDIX B: LASER MAINTENANCE}

\section{Deionization of the Cooling System (Weekly)}

Run the laser's cooling system for approximately 30 minutes every week to avoid the buildup of ions in the lasing cavity. If gone unchecked, the buildup of ions on the Nd:YAG rod and flash lamp will be burnt during laser operation and cause a significant decrease in laser energy output. If the laser will be stored for an extended period, drain and dry the cooling system with compressed air prior to storage.

There should be a 50/50 split in flow between the deionization cartridge and the return bypass line to the cooling reservoir. A clamp has been placed on the bypass line to restrict flow and achieve an even flow between the filter and bypass. Over time, as the filter ages and fills, the clamps may need adjustment to maintain an even flow rate. If the laser power supply kicks off when initiating the standby mode, illuminating the water flow indicator on the power supply front panel, the bypass clamp may need to be loosened to allow greater water flow rate through the laser head.

\section{Optics Cleaning (Monthly)}

Check the laser optics monthly for dust, burn marks, or scratches and clean with lens tissue and deionized or distilled water, replace if necessary. To minimize dust accumulation, the aperture on the laser should remain closed when the laser is not in use.

\section{Maximum Energy (Monthly)}

A decrease in maximum output energy is a key indicator that the laser is in need of maintenance, the output for each laser should be checked and recorded monthly to track laser performance and detect any decreases in energy. Coherent's Field Max II TOP energy meter and J-50MB-YAG sensor are currently used to monitor the laser's performance; however, any meter and sensor capable of measuring 200+ $\mathrm{mJ} /$ pulse at 532 $\mathrm{nm}$ will suffice. 


\section{APPENDIX C: COMBUSTION VESSEL FLOW PROFILES}

Combustion Vessel Mean Velocity Profiles

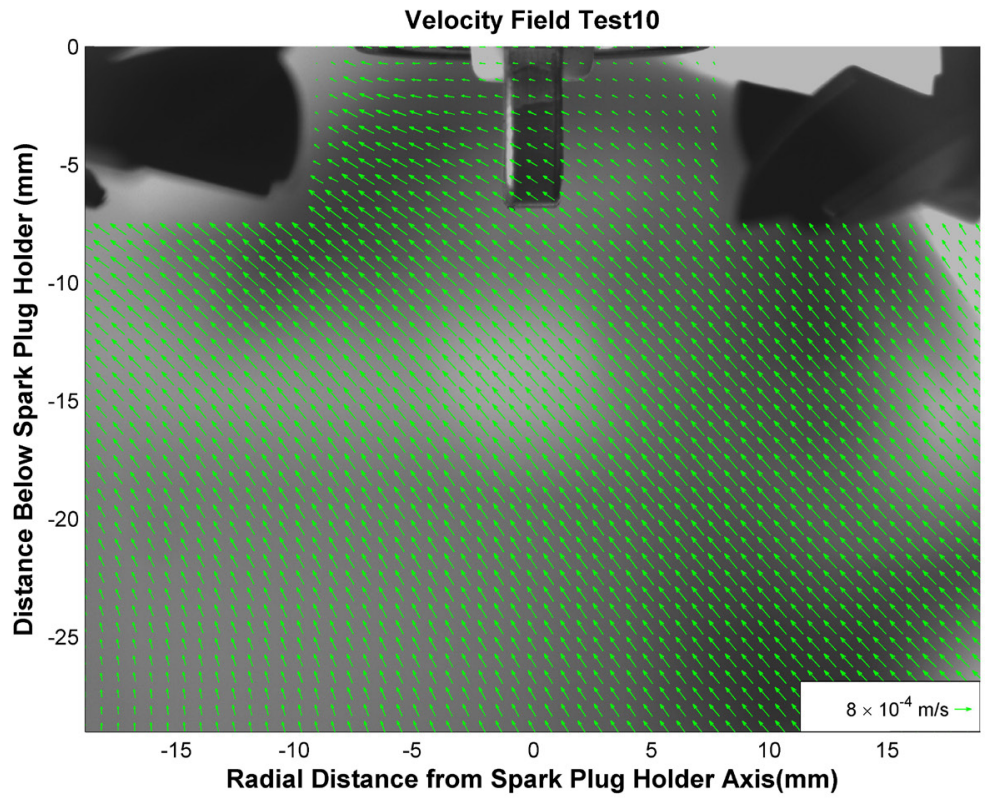

Figure C.1 Mean velocity vector profile for Test 10 . 


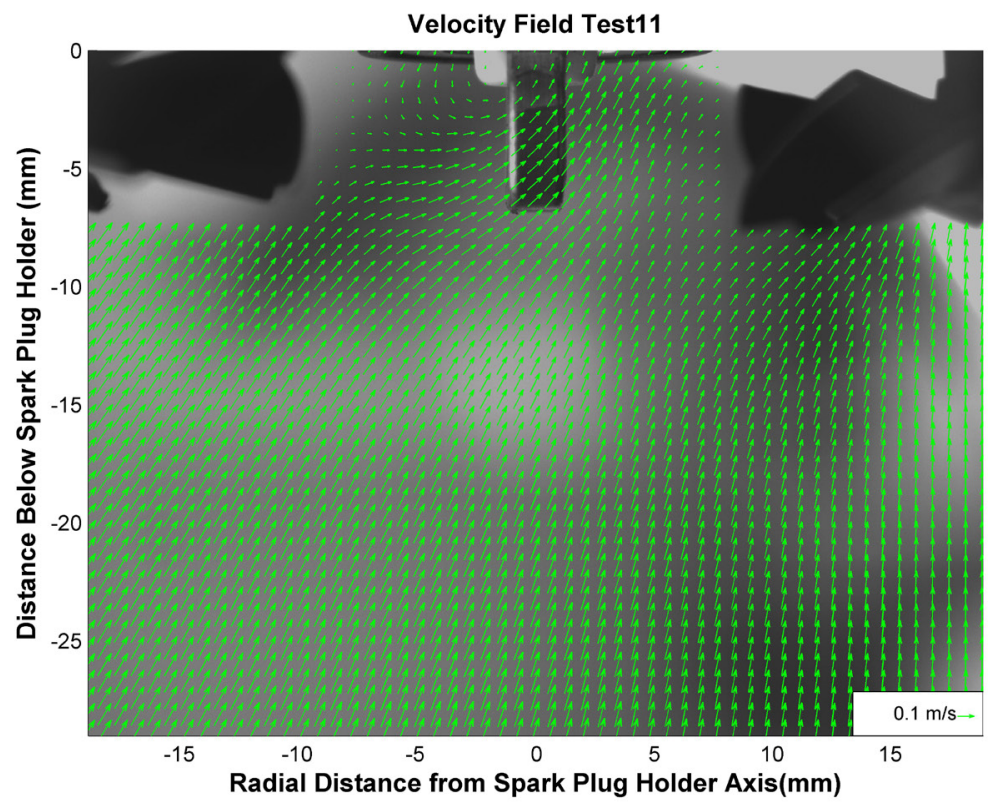

Figure C.2 Mean velocity vector profile for Test 11 .

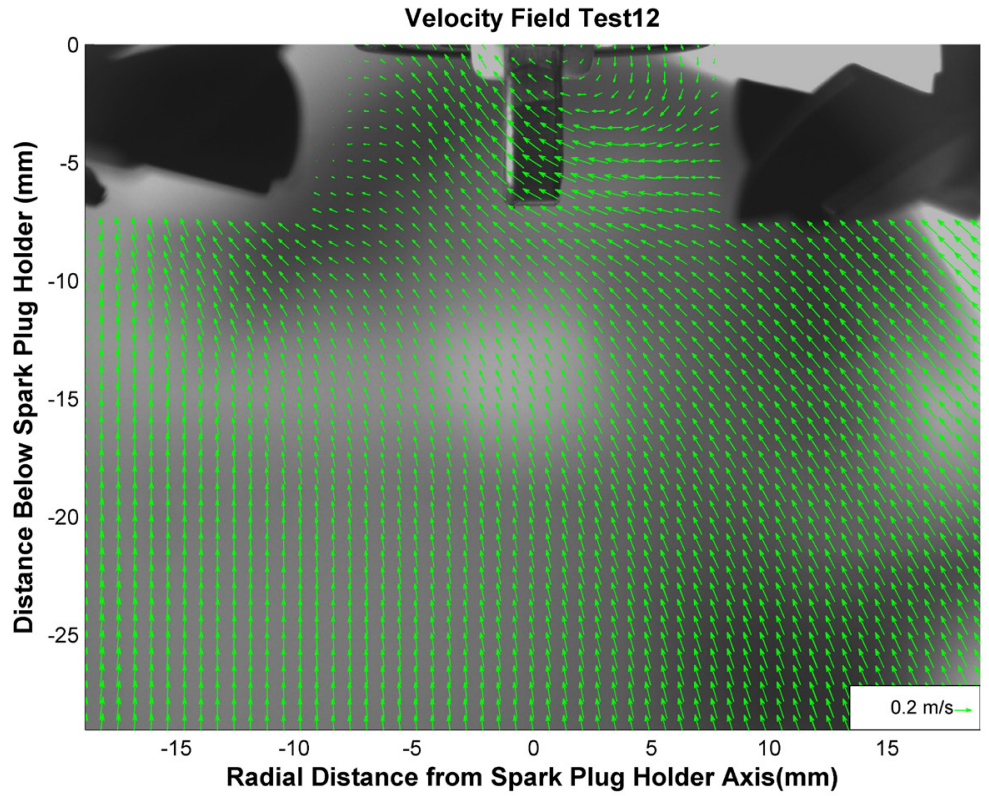

Figure C.3 Mean velocity vector profile for Test 12 . 


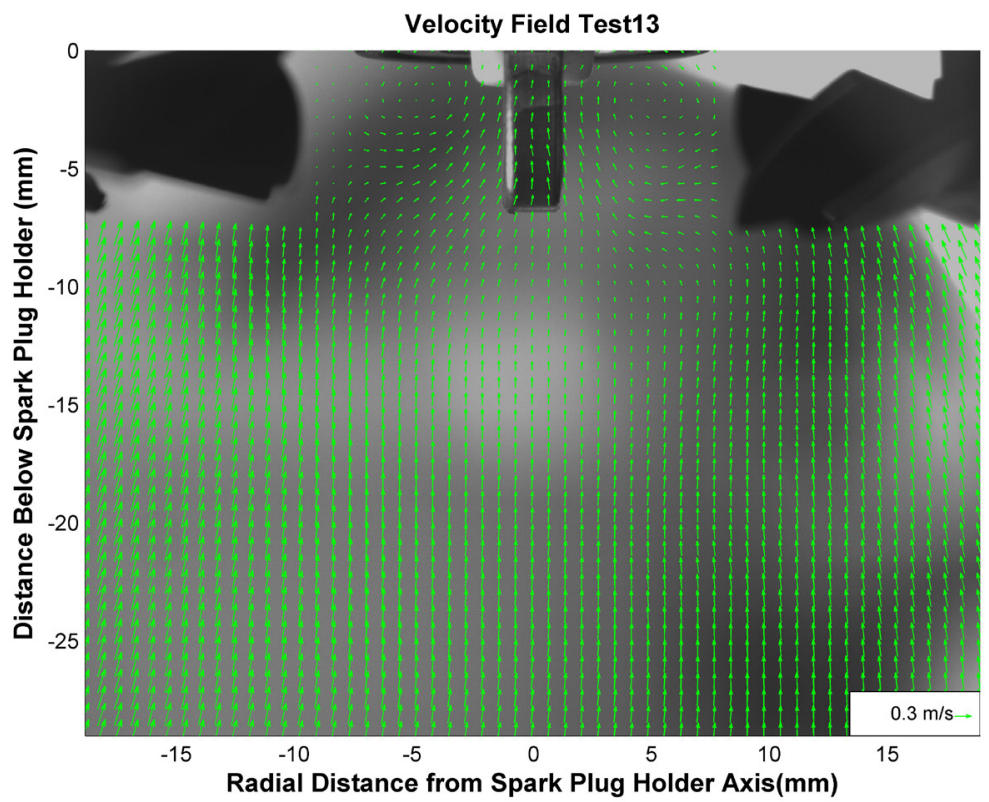

Figure C.4 Mean velocity vector profile for Test 13 .

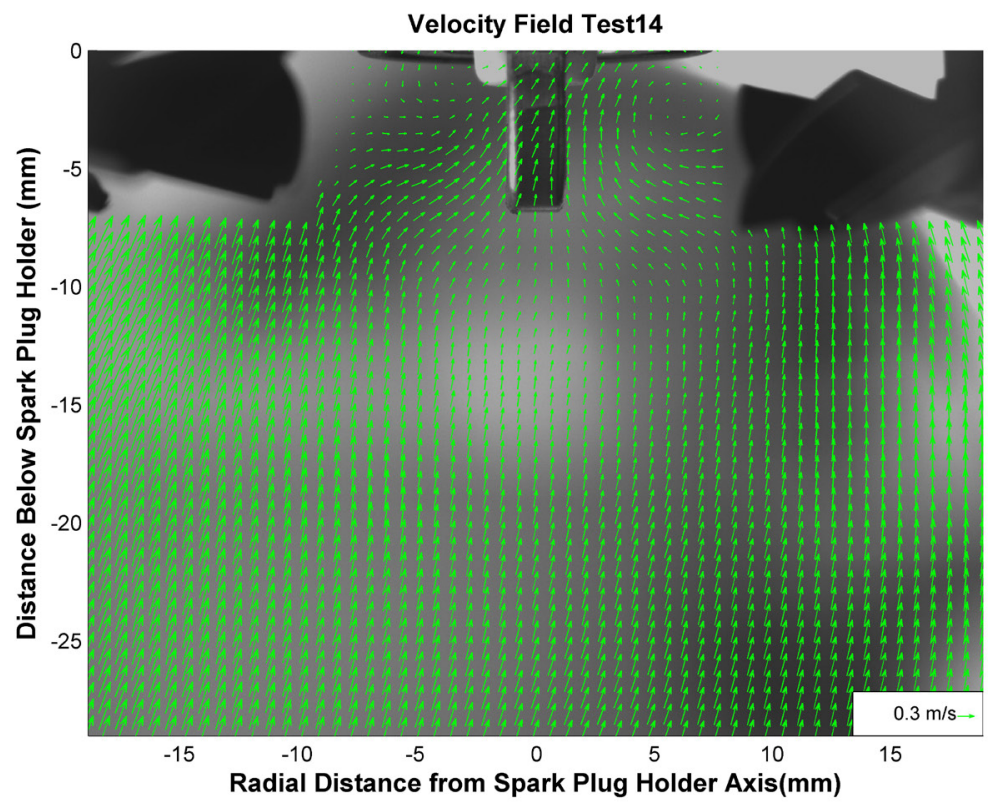

Figure C.5 Mean velocity vector profile for Test 14 . 


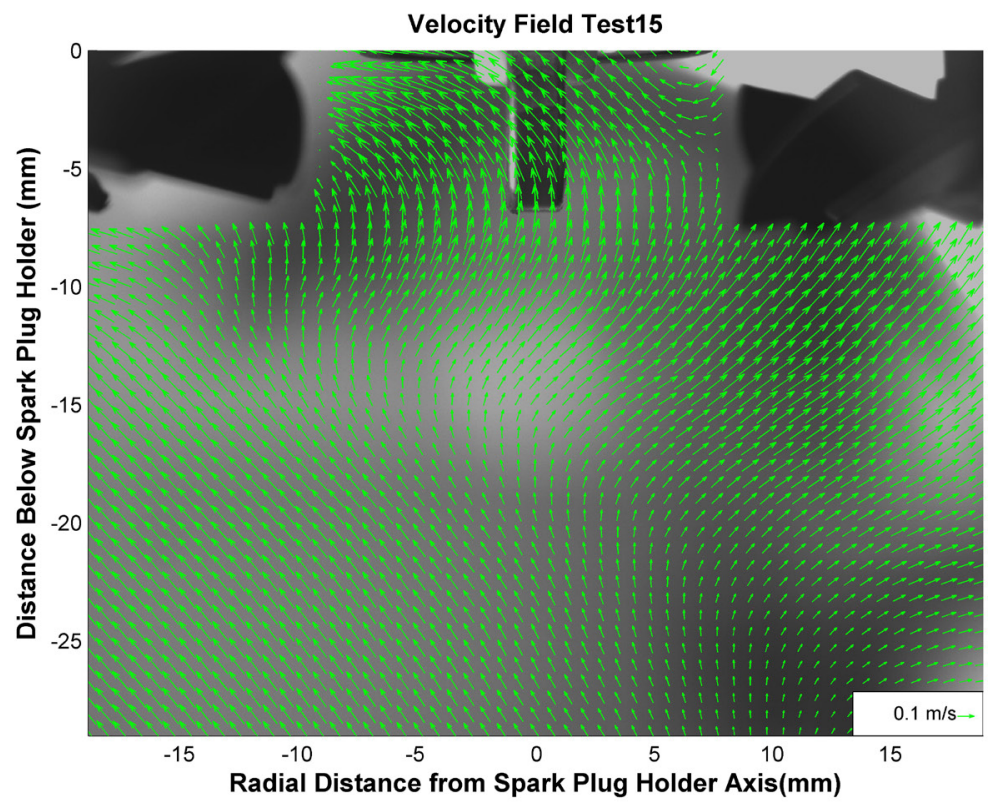

Figure C.6 Mean velocity vector profile for Test 15 .

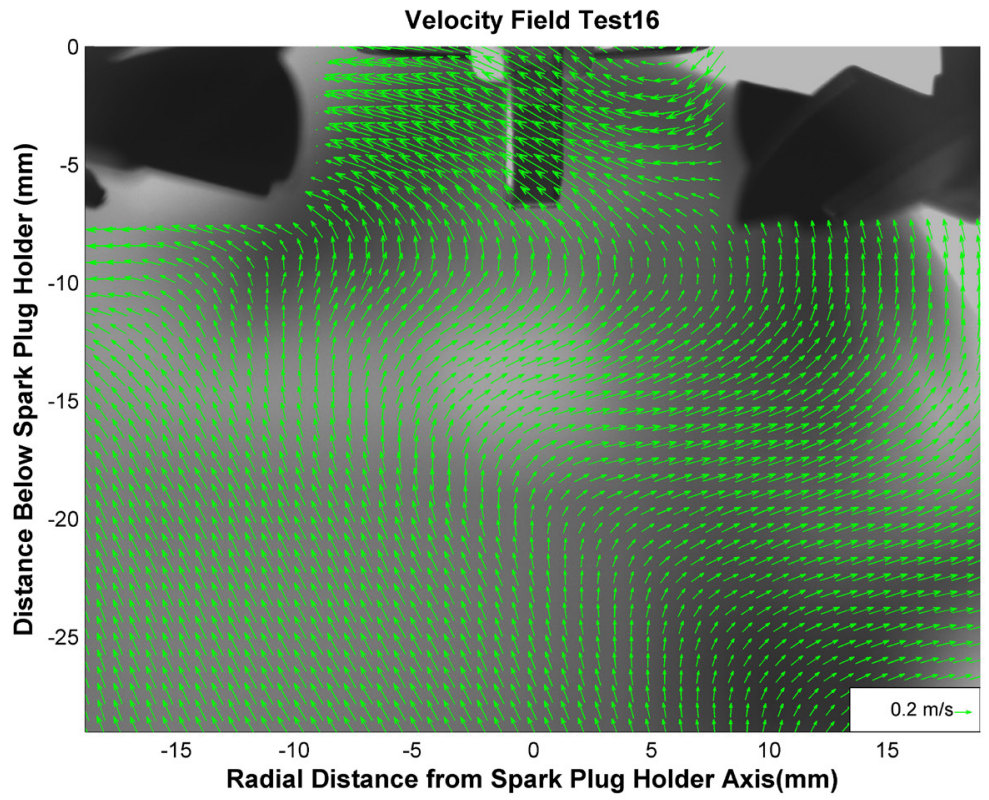

Figure C.7 Mean velocity vector profile for Test 16 . 


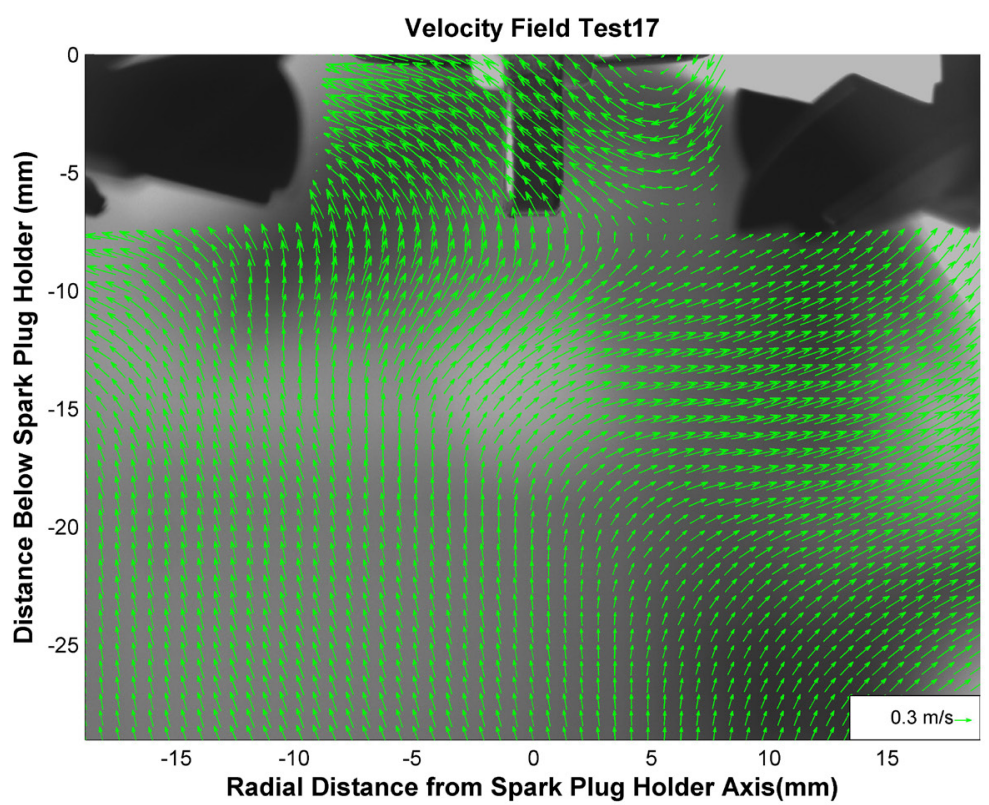

Figure C.8 Mean velocity vector profile for Test 17 .

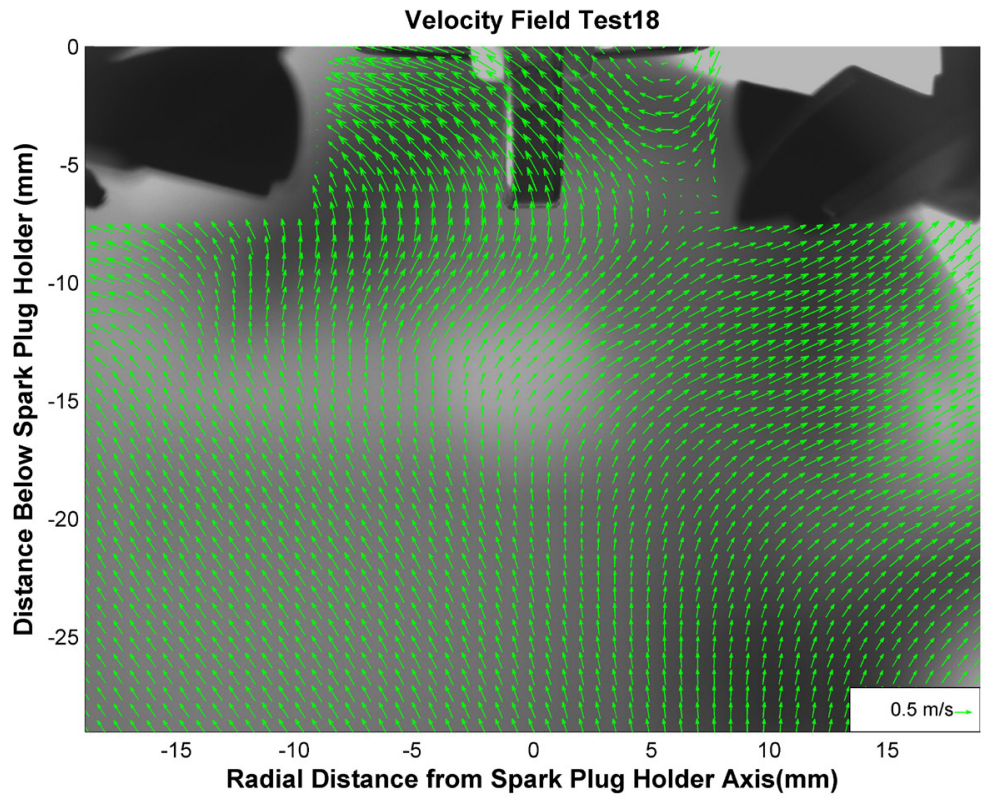

Figure C.9 Mean velocity vector profile for Test 18 . 


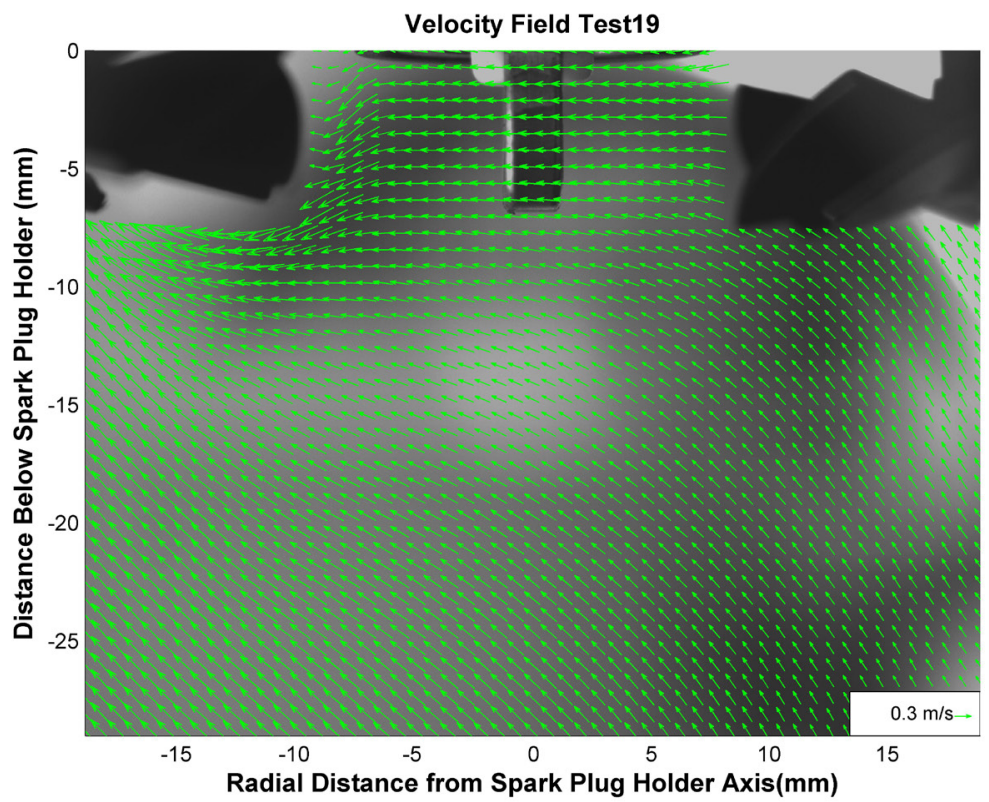

Figure C.10 Mean velocity vector profile for Test 19.

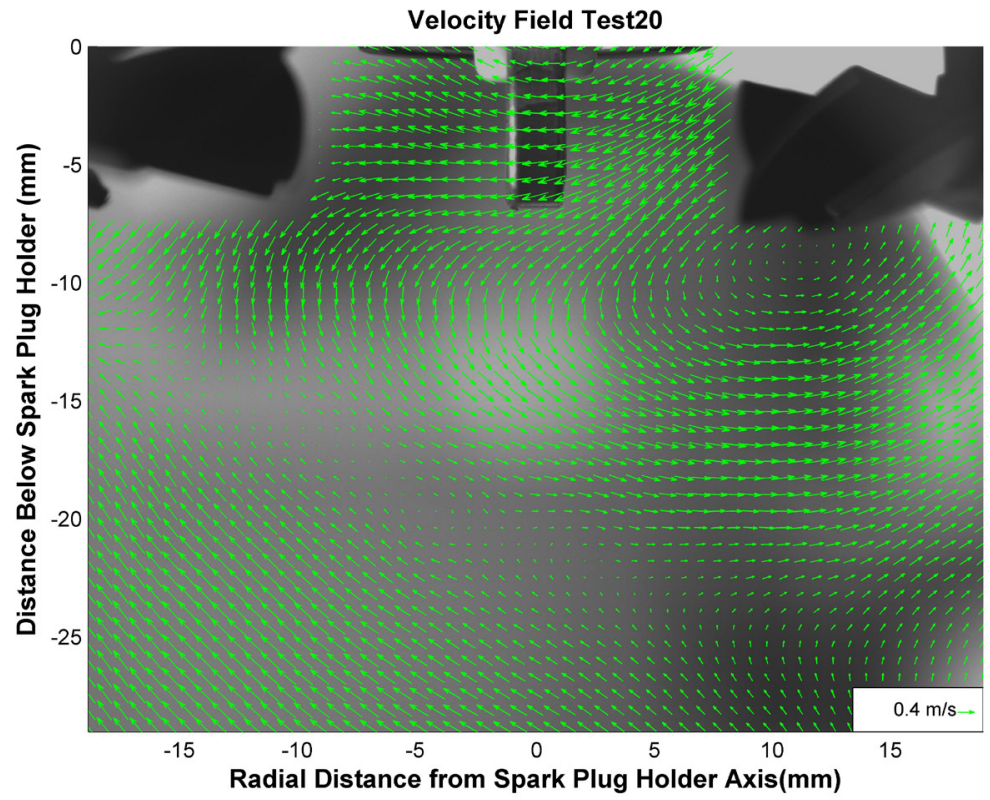

Figure C.11 Mean velocity vector profile for Test 20 . 


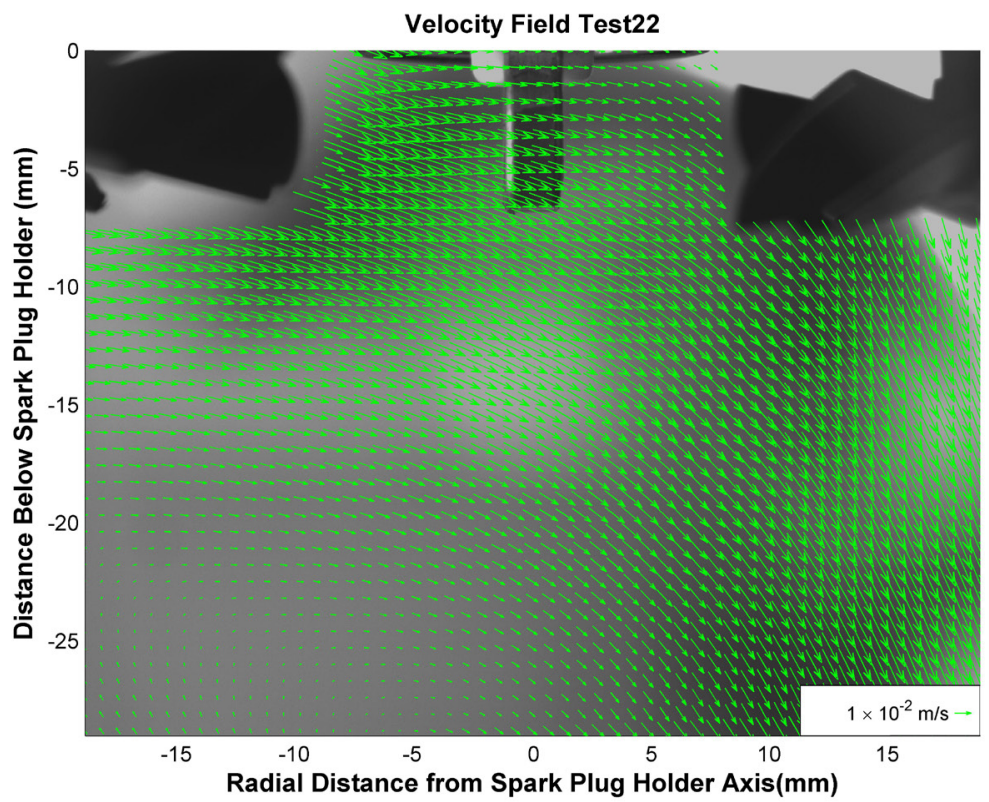

Figure C.12 Mean velocity vector profile for Test 22 .

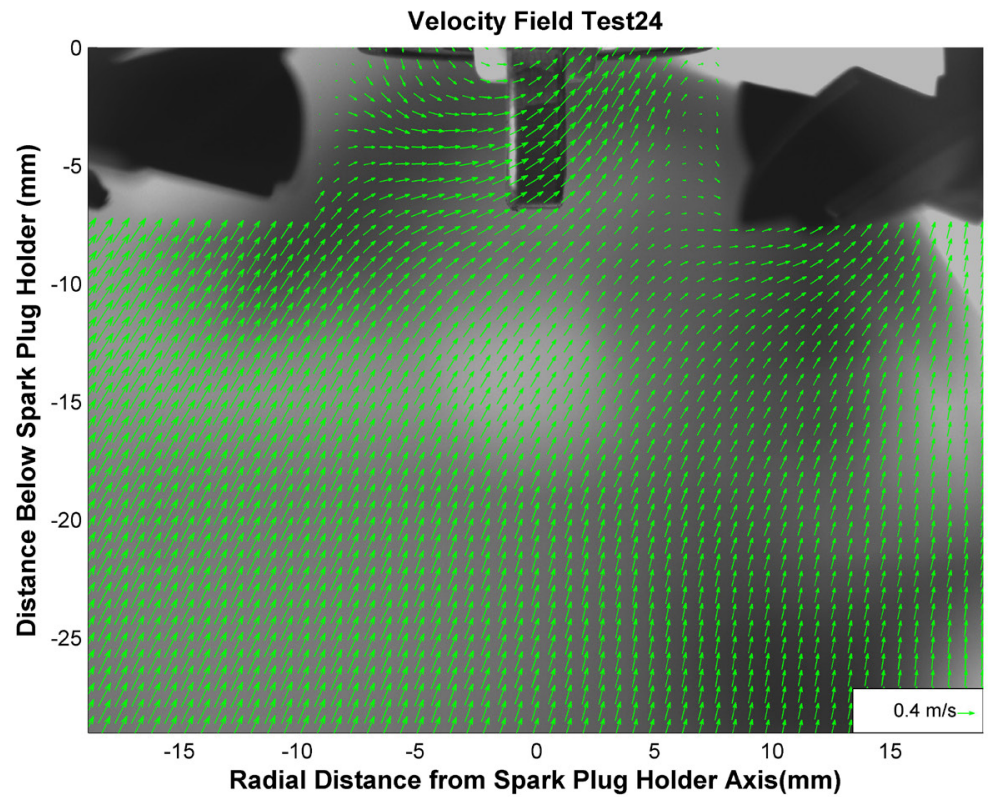

Figure C.13 Mean velocity vector profile for Test 24 . 


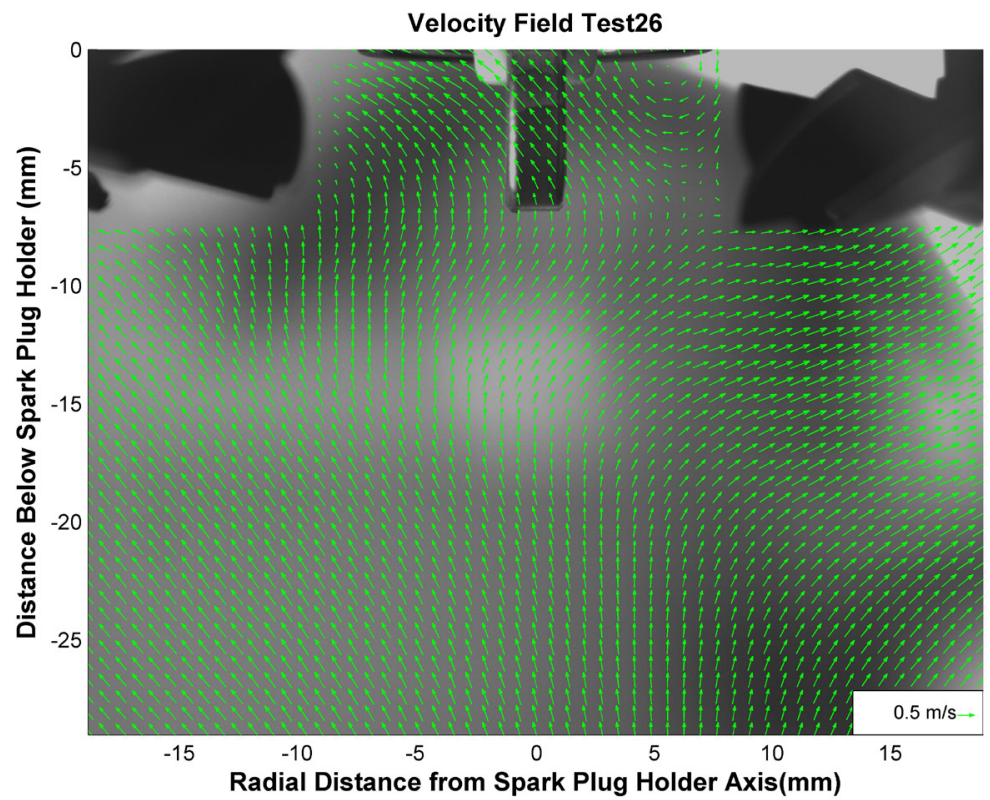

Figure C.14 Mean velocity vector profile for Test 26. 


\section{Combustion Vessel Turbulent Velocity Profiles}

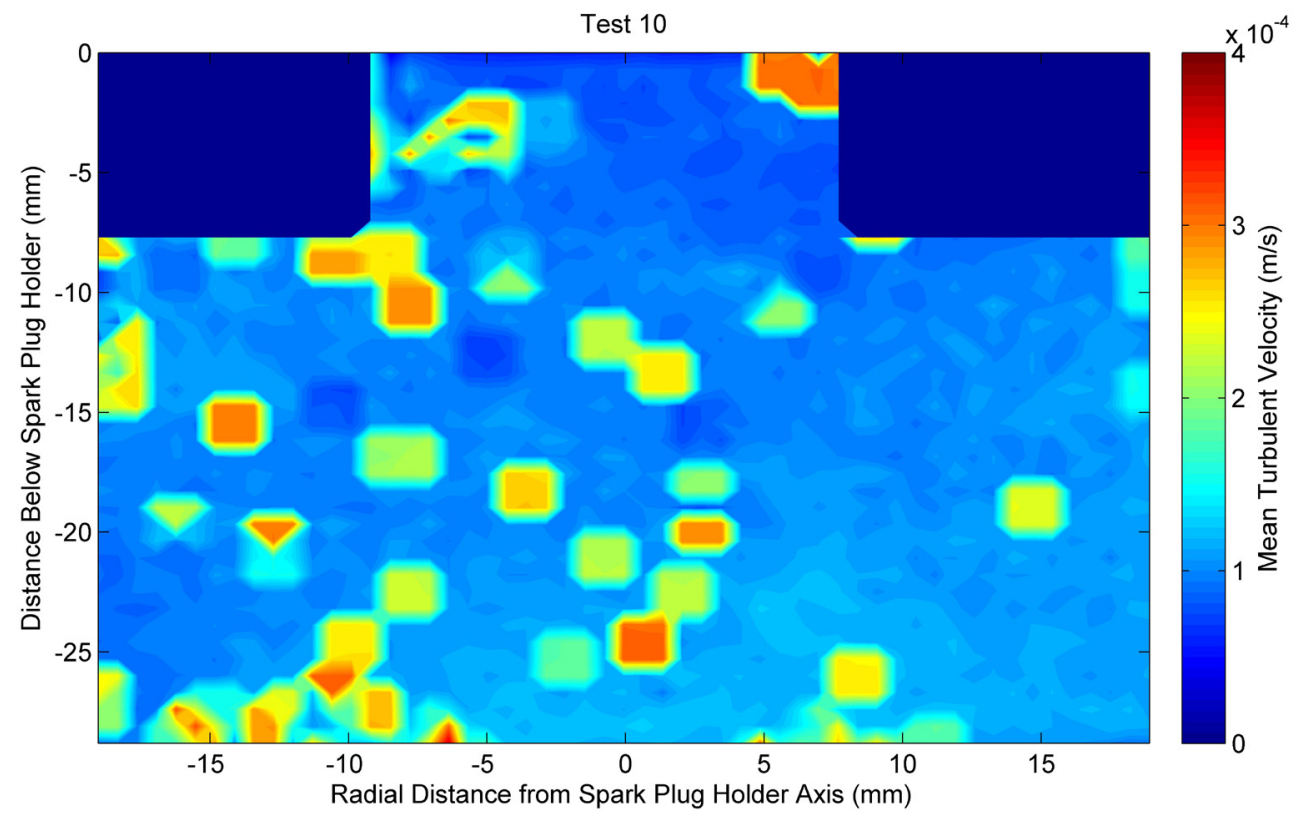

Figure C.15 Mean turbulent velocity profile for Test 10 . 


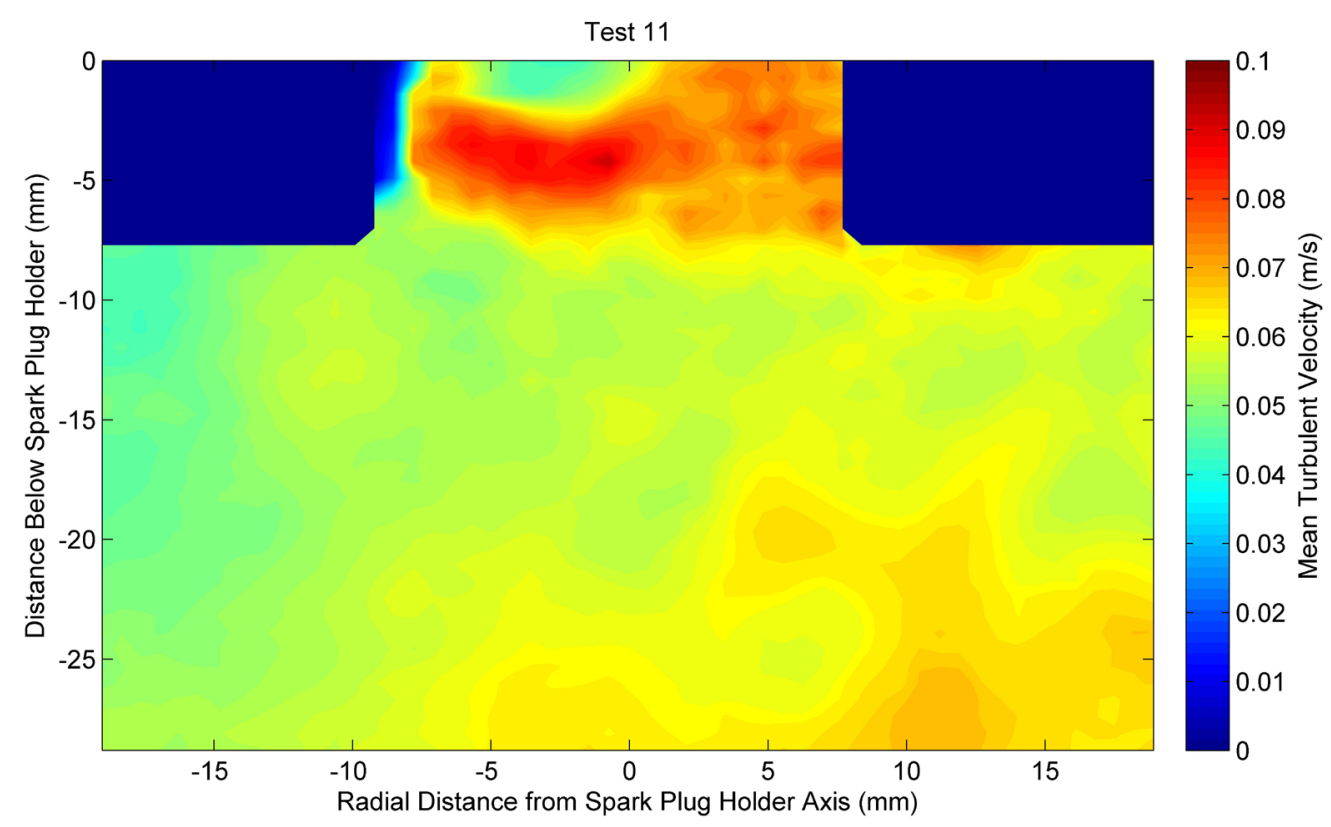

Figure C.16 Mean turbulent velocity profile for Test 11.

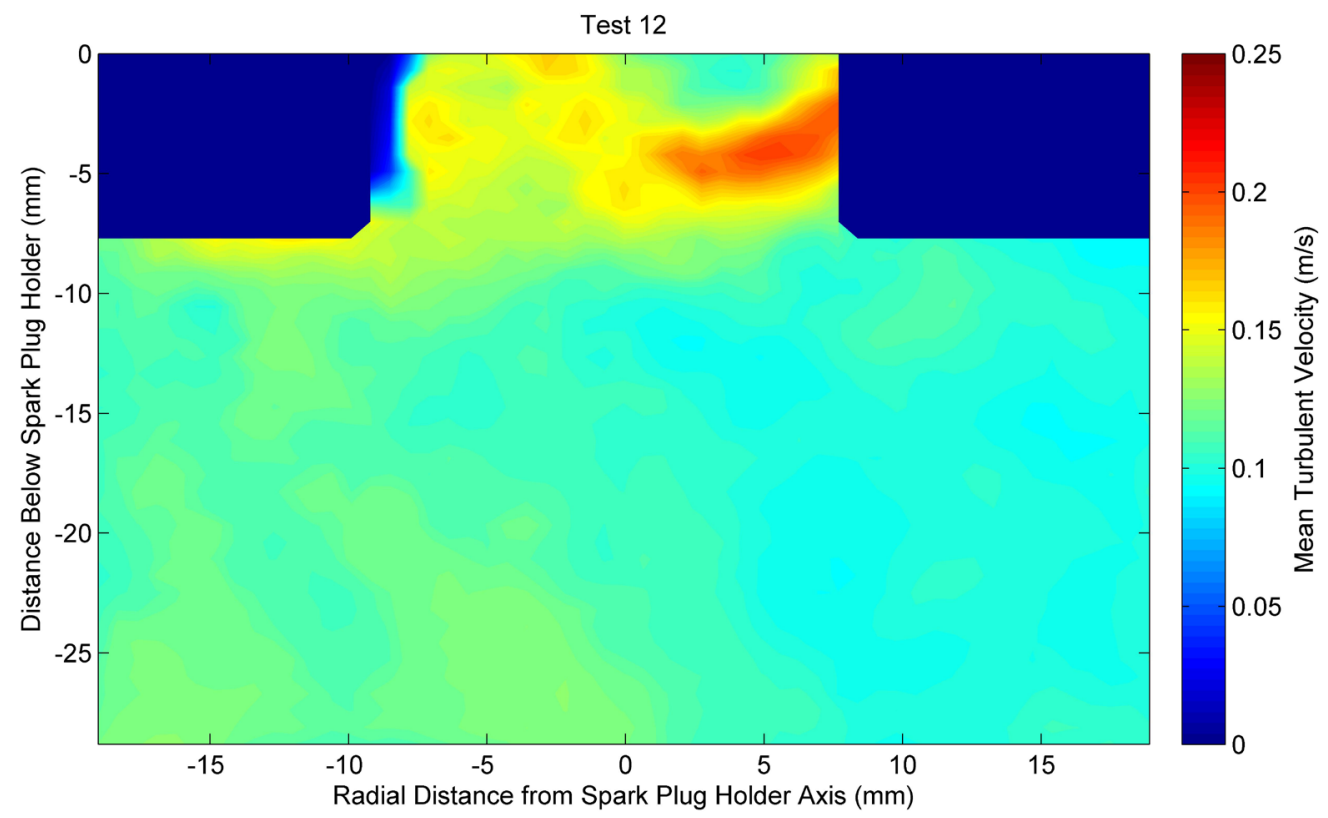

Figure C.17 Mean turbulent velocity profile for Test 12 . 


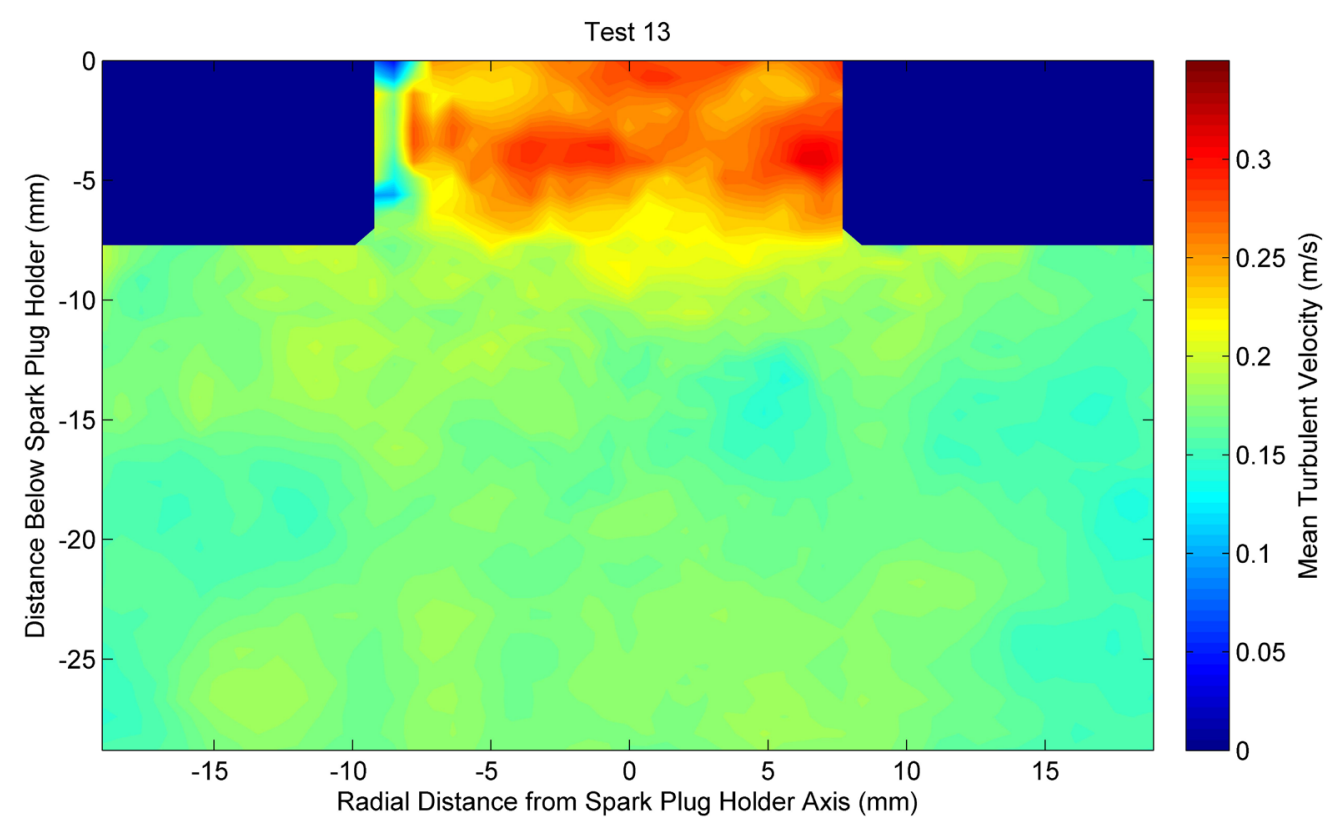

Figure C.18 Mean turbulent velocity profile for Test 13.

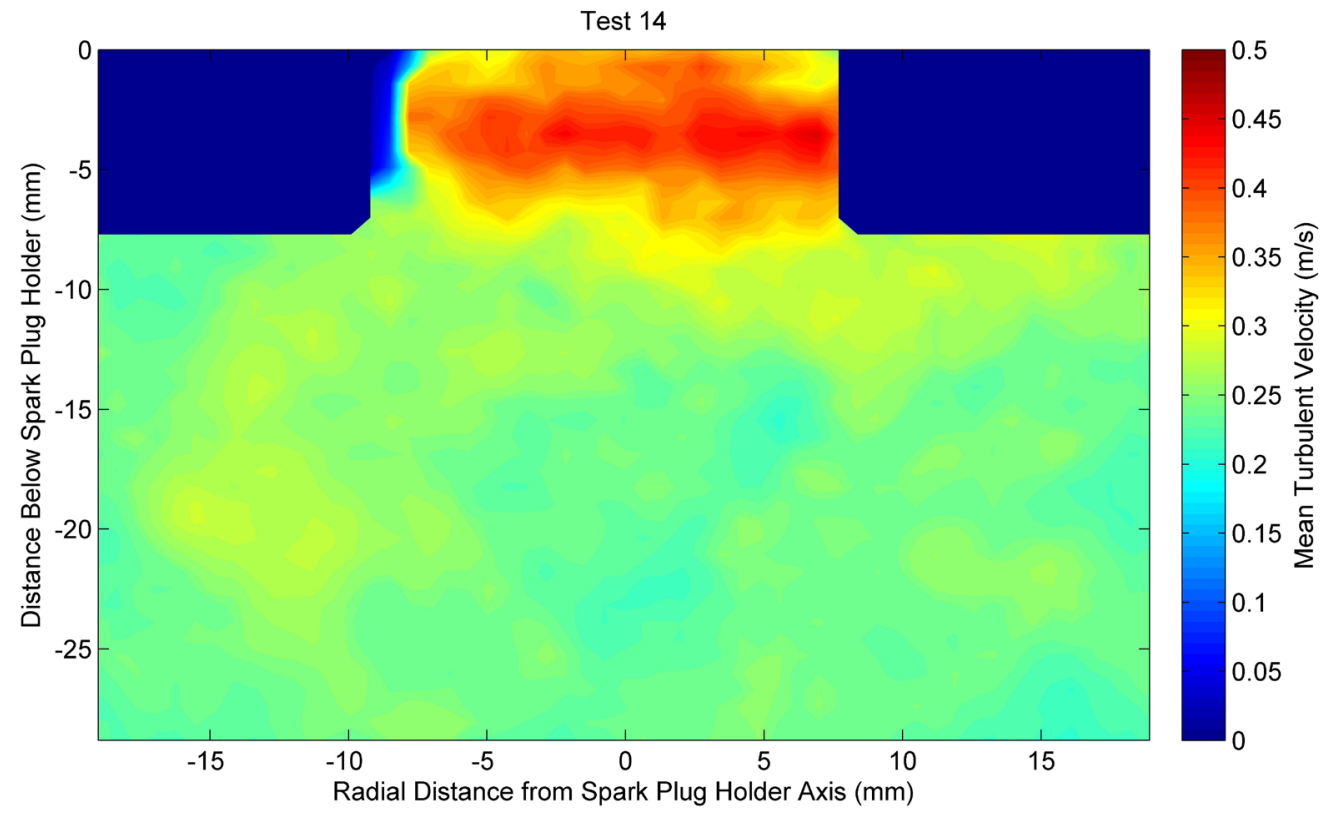

Figure C.19 Mean turbulent velocity profile for Test 14 . 


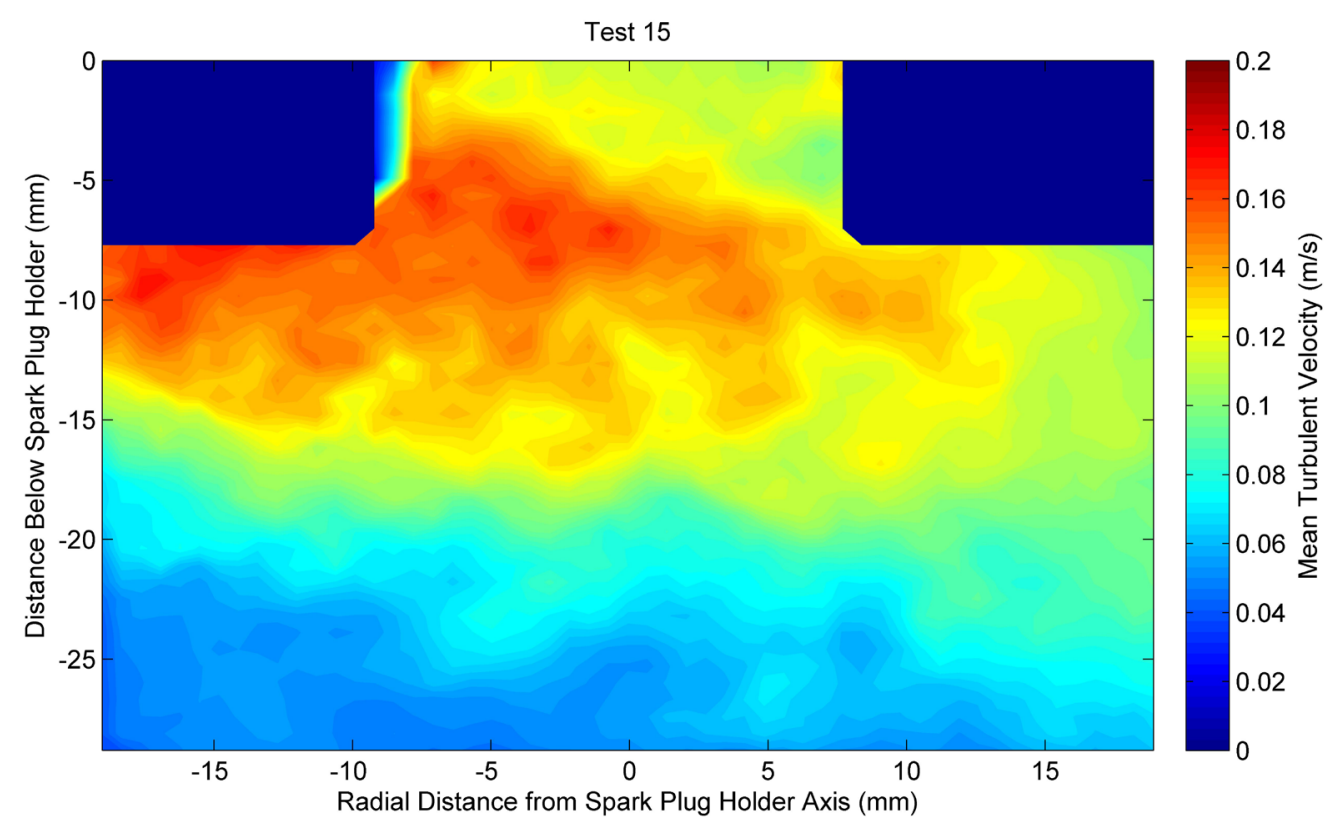

Figure C.20 Mean turbulent velocity profile for Test 15 .

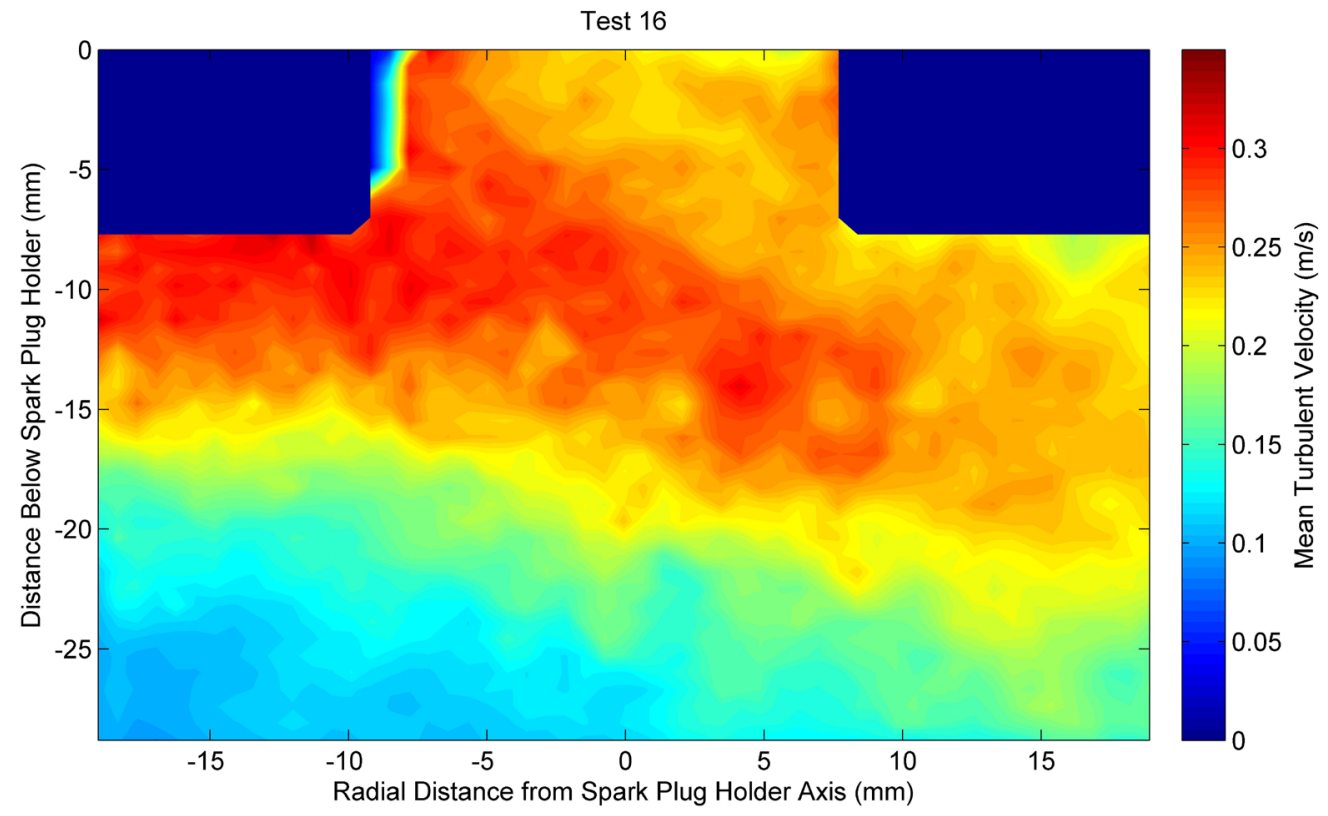

Figure C.21 Mean turbulent velocity profile for Test 16. 


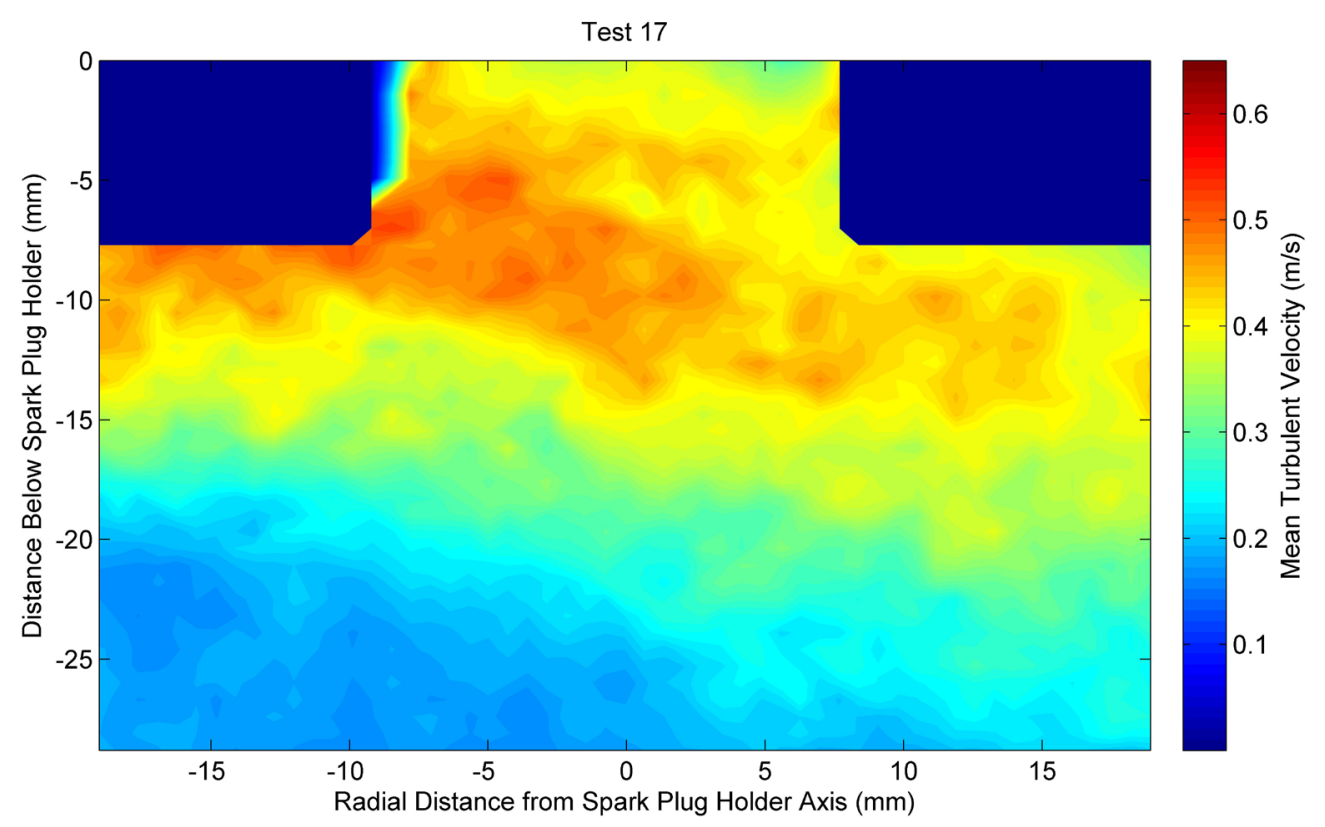

Figure C.22 Mean turbulent velocity profile for Test 17 .

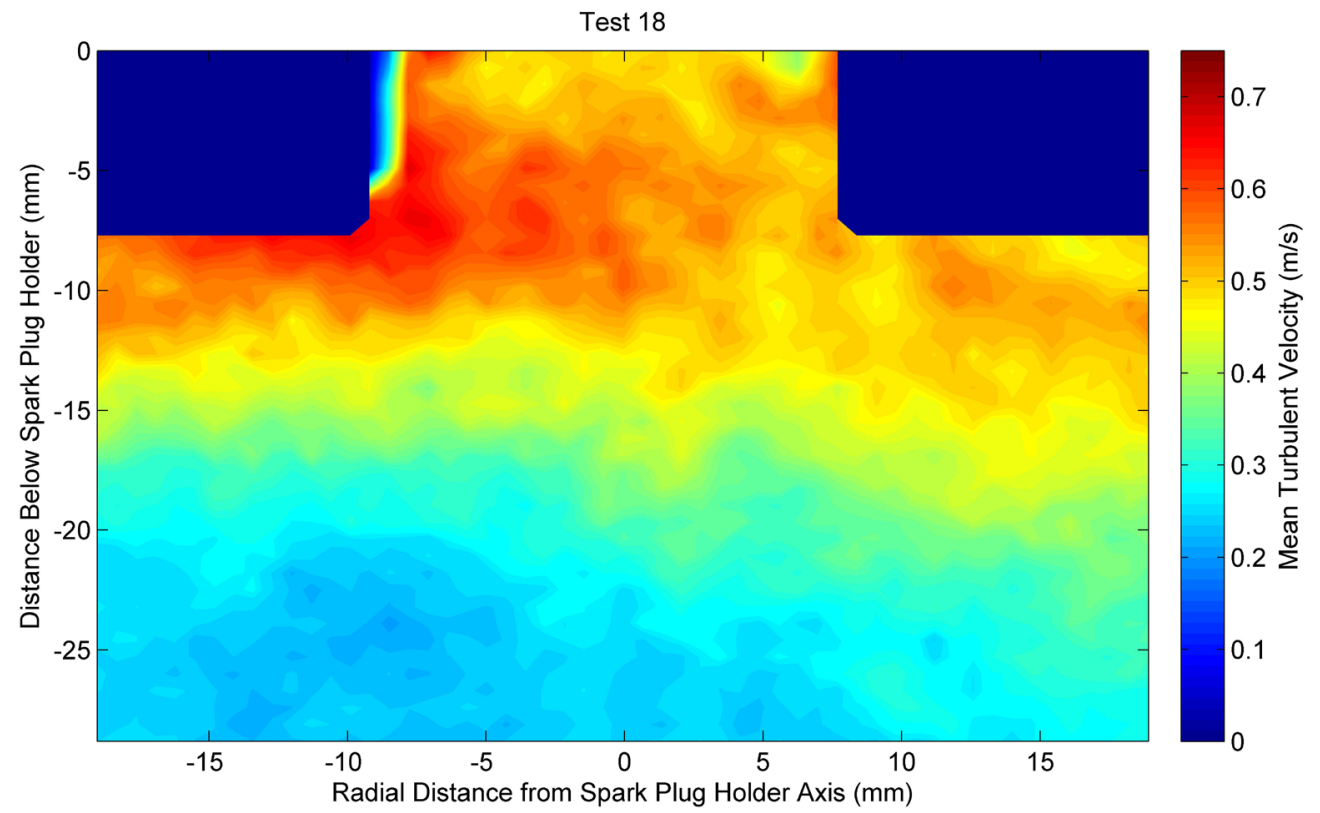

Figure C.23 Mean turbulent velocity profile for Test 18 . 


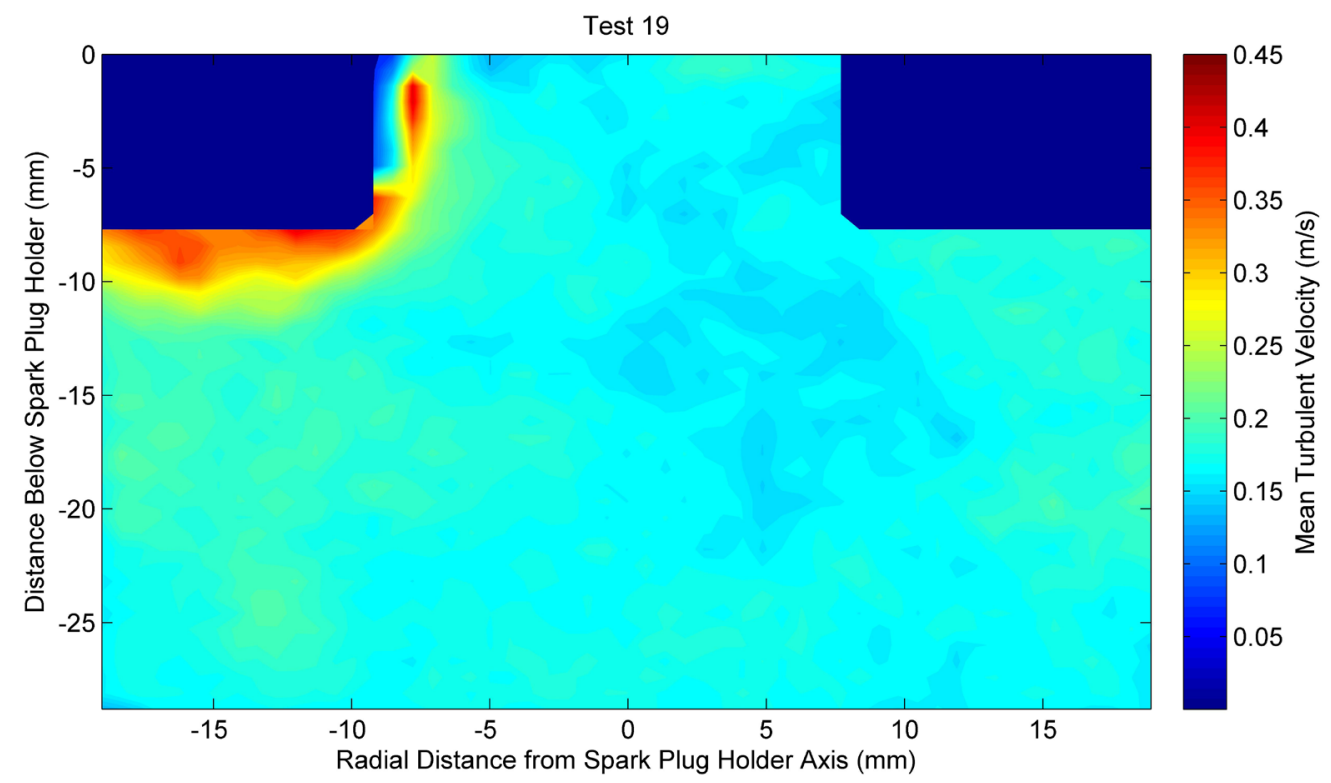

Figure C.24 Mean turbulent velocity profile for Test 19.

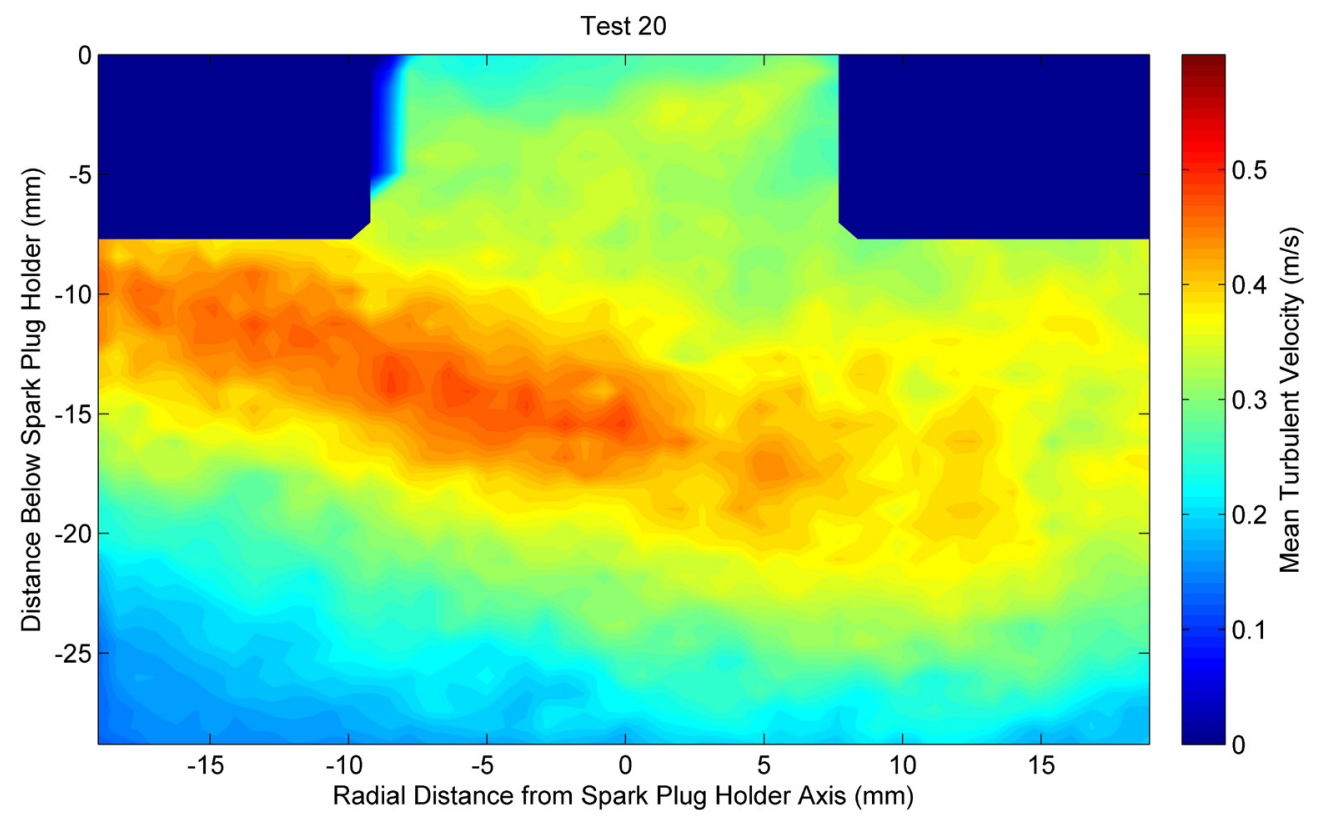

Figure C. 25 Mean turbulent velocity profile for Test 20. 


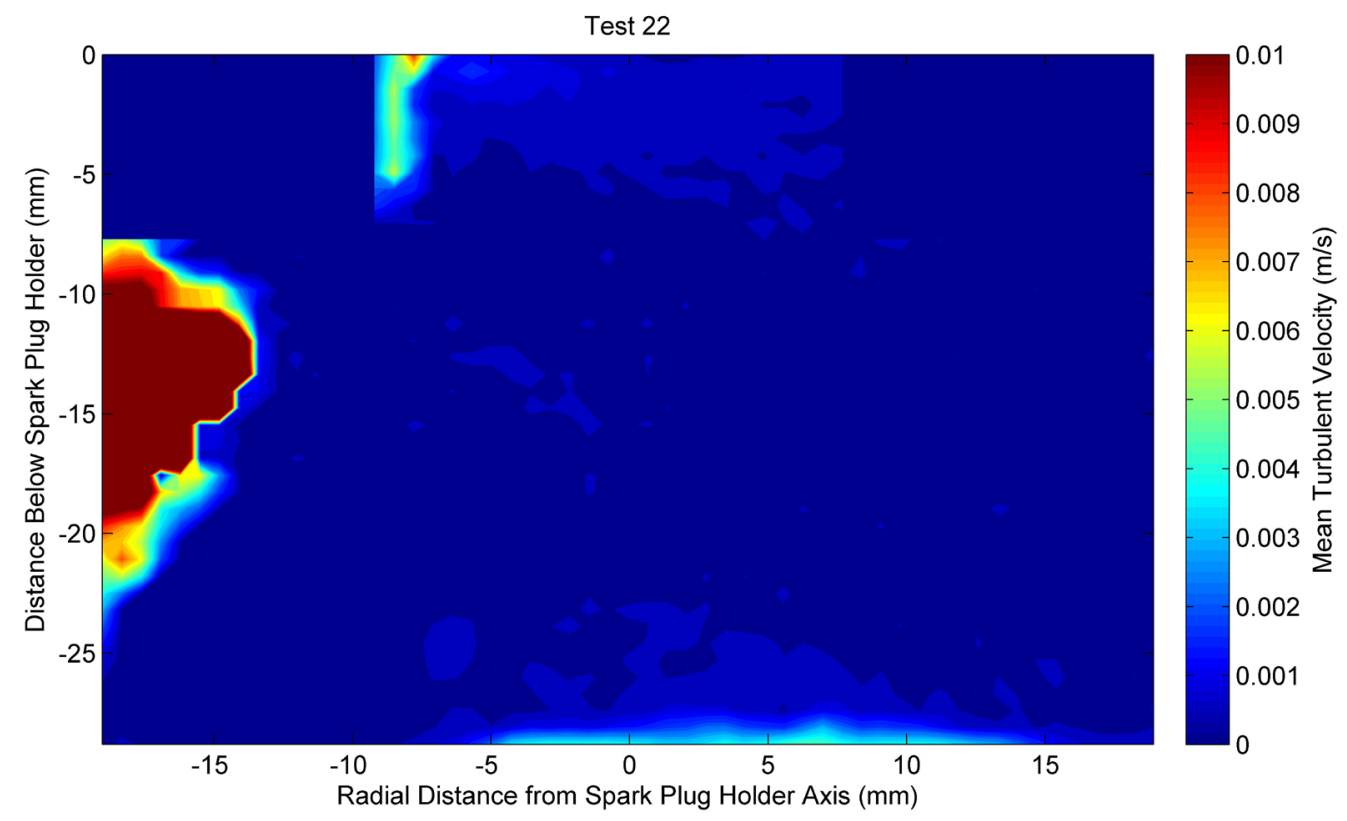

Figure C.26 Mean turbulent velocity profile for Test 22.

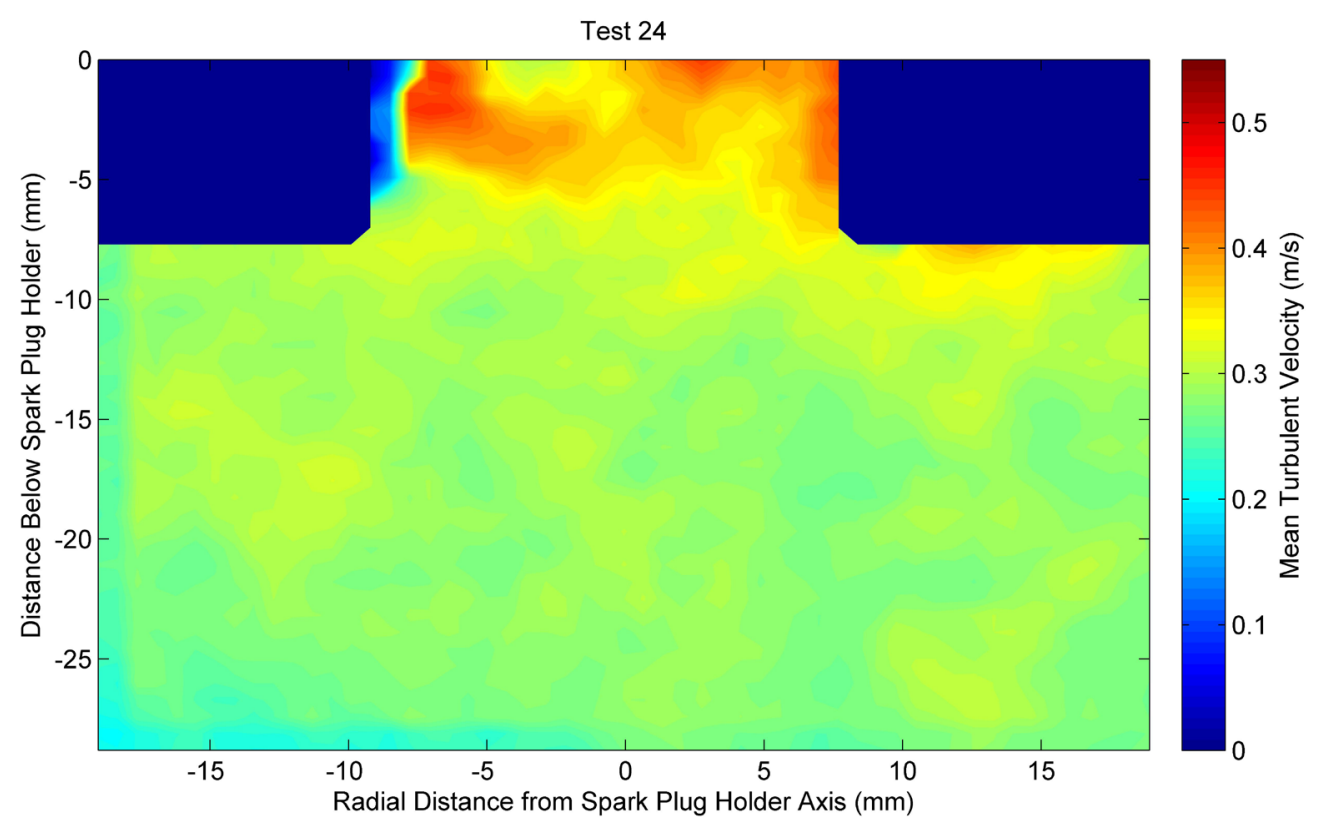

Figure C.27 Mean turbulent velocity profile for Test 24 . 


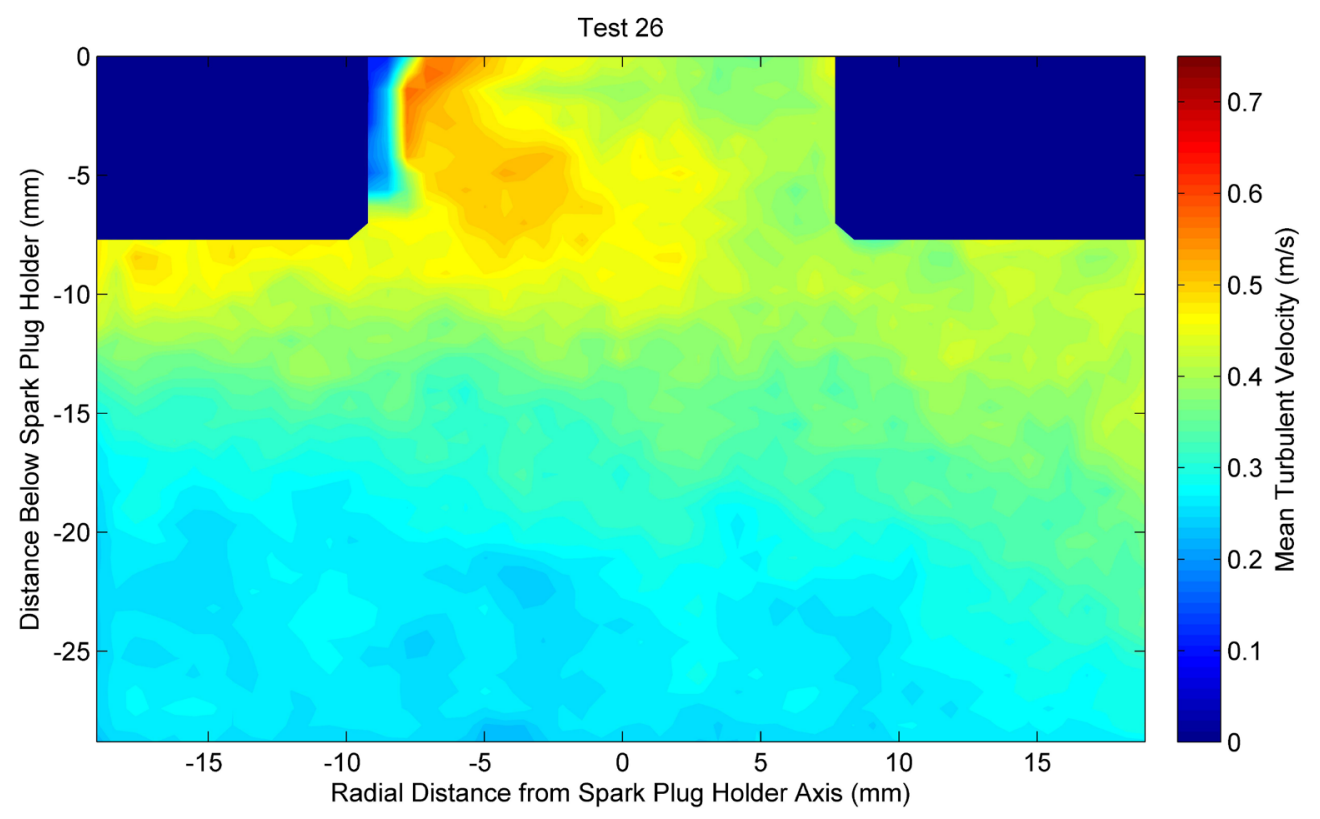

Figure C.28 Mean turbulent velocity profile for Test 26 . 